University of Tennessee Health Science Center UTHSC Digital Commons

\title{
$5-2011$
}

\section{Effect of Occlusion, Directionality and Age on Horizontal Localization}

Lynzee Nicole Alworth

University of Tennessee Health Science Center

Follow this and additional works at: https://dc.uthsc.edu/dissertations

Part of the Analytical, Diagnostic and Therapeutic Techniques and Equipment Commons, Speech and Hearing Science Commons, and the Speech Pathology and Audiology Commons

\section{Recommended Citation}

Alworth, Lynzee Nicole , "Effect of Occlusion, Directionality and Age on Horizontal Localization" (2011). Theses and Dissertations (ETD). Paper 17. http://dx.doi.org/10.21007/etd.cghs.2011.0012. 


\title{
Effect of Occlusion, Directionality and Age on Horizontal Localization
}

\begin{abstract}
Localization acuity of a given listener is dependent upon the ability discriminate between interaural time and level disparities. Interaural time differences are encoded by low frequency information whereas interaural level differences are encoded by high frequency information. Much research has examined effects of hearing aid microphone technologies and occlusion separately and prior studies have not evaluated age as a factor in localization acuity. Open-fit hearing instruments provide new earmold technologies and varying microphone capabilities; however, these instruments have yet to be evaluated with regard to horizontal localization acuity.
\end{abstract}

Thus, the purpose of this study is to examine the effects of microphone configuration, type of dome in open-fit hearing instruments, and age on the horizontal localization ability of a given listener. Thirty adults participated in this study and were grouped based upon hearing sensitivity and age (young normal hearing, $>50$ years normal hearing, $>50$ hearing impaired). Each normal hearing participant completed one localization experiment (unaided/unamplified) where they listened to the stimulus "Baseball" and selected the point of origin. Hearing impaired listeners were fit with the same two receiver-in-the-ear hearing aids and same dome types, thus controlling for microphone technologies, type of dome, and fitting between trials. Hearing impaired listeners completed a total of 7 localization experiments (unaided/unamplified; open dome: omnidirectional, adaptive directional, fixed directional; micromold: omnidirectional, adaptive directional, fixed directional).

Overall, results of this study indicate that age significantly affects horizontal localization ability as younger adult listeners with normal hearing made significantly fewer localization errors than older adult listeners with normal hearing. Also, results revealed a significant difference in performance between dome type; however, upon further examination was not significant. Therefore, results examining type of dome should be viewed with caution. Results examining microphone configuration and microphone configuration by dome type were not significant. Moreover, results evaluating performance relative to unaided (unamplified) were not significant. Taken together, these results suggest open-fit hearing instruments, regardless of microphone or dome type, do not degrade horizontal localization acuity within a given listener relative to their 'older aged' normal hearing counterparts in quiet environments.

\section{Document Type}

Dissertation

Degree Name

Doctor of Philosophy (PhD)

Program

Speech and Hearing Science

Research Advisor

Patrick N. Plyler, Ph.D.

Keywords

Age, Directionality, Localization, Occlusion

Subject Categories

Analytical, Diagnostic and Therapeutic Techniques and Equipment | Medicine and Health Sciences | 
Speech and Hearing Science I Speech Pathology and Audiology

This dissertation is available at UTHSC Digital Commons: https://dc.uthsc.edu/dissertations/17 


\title{
EFFECT OF OCCLUSION, DIRECTIONALITY AND AGE ON HORIZONTAL
} LOCALIZATION

\author{
A Dissertation \\ Presented for the \\ The Graduate Studies Council \\ The University of Tennessee \\ Health Science Center
}

\begin{abstract}
In Partial Fulfillment
Of the Requirements for the Degree

Doctor of Philosophy

From The University of Tennessee
\end{abstract}

By

Lynzee Nicole Alworth

May 2011 
Copyright (C) 2011 by Lynzee Nicole Alworth. All rights reserved. 


\section{ACKNOWLEDGEMENTS}

I would like to take this opportunity to thank my parents, family and friends for their continued encouragement, patience, and support during this intensive process otherwise known as the dissertation experience. Each played a crucial role and provided me with the confidence necessary to complete this challenging series of events in my life. For this, words cannot begin to express my gratitude.

To my amazing advisor, mentor and friend, Dr. Patrick Plyler. I would like to thank him for his continued guidance and encouragement throughout my doctoral program. The inspiration and confidence he provided made even the largest undertaking enjoyable.

I am also very thankful to Dr. Patti Johnstone for her time, assistance, and enthusiasm during this experience. Her continued support was invaluable throughout this endeavor. Also, to the clinical faculty (Dr. Beth Humphrey, Dr. Nancy Schay, Dr. Kelly Yeager, and Dr. Erin Plyler) whose clinical insight, continued encouragement, and guidance were irreplaceable. 


\begin{abstract}
Localization acuity of a given listener is dependent upon the ability discriminate between interaural time and level disparities. Interaural time differences are encoded by low frequency information whereas interaural level differences are encoded by high frequency information. Much research has examined effects of hearing aid microphone technologies and occlusion separately and prior studies have not evaluated age as a factor in localization acuity. Open-fit hearing instruments provide new earmold technologies and varying microphone capabilities; however, these instruments have yet to be evaluated with regard to horizontal localization acuity.

Thus, the purpose of this study is to examine the effects of microphone configuration, type of dome in open-fit hearing instruments, and age on the horizontal localization ability of a given listener. Thirty adults participated in this study and were grouped based upon hearing sensitivity and age (young normal hearing, $>50$ years normal hearing, $>50$ hearing impaired). Each normal hearing participant completed one localization experiment (unaided/unamplified) where they listened to the stimulus "Baseball" and selected the point of origin. Hearing impaired listeners were fit with the same two receiver-in-the-ear hearing aids and same dome types, thus controlling for microphone technologies, type of dome, and fitting between trials. Hearing impaired listeners completed a total of 7 localization experiments (unaided/unamplified; open dome: omnidirectional, adaptive directional, fixed directional; micromold: omnidirectional, adaptive directional, fixed directional).

Overall, results of this study indicate that age significantly affects horizontal localization ability as younger adult listeners with normal hearing made significantly fewer localization errors than older adult listeners with normal hearing. Also, results revealed a significant difference in performance between dome type; however, upon further examination was not significant. Therefore, results examining type of dome should be viewed with caution. Results examining microphone configuration and microphone configuration by dome type were not significant. Moreover, results evaluating performance relative to unaided (unamplified) were not significant. Taken together, these results suggest open-fit hearing instruments, regardless of microphone or dome type, do not degrade horizontal localization acuity within a given listener relative to their 'older aged' normal hearing counterparts in quiet environments.
\end{abstract}




\section{TABLE OF CONTENTS}

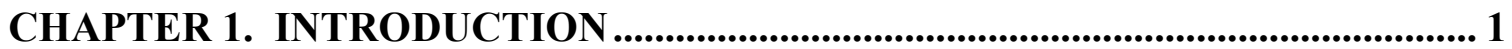

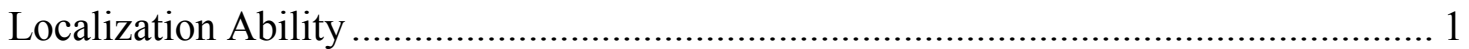

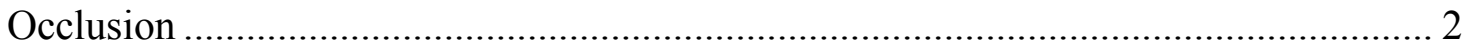

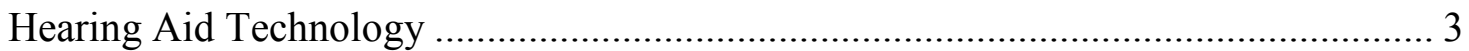

Microphone Placement ……………………………........................................ 3

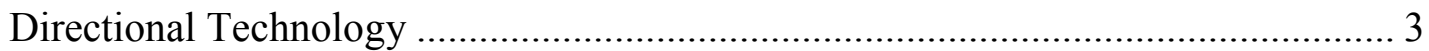

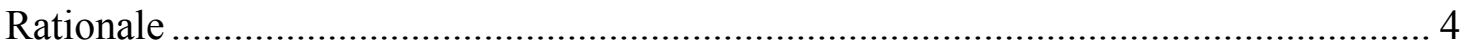

CHAPTER 2. REVIEW OF LITERATURE ....................................................... 6

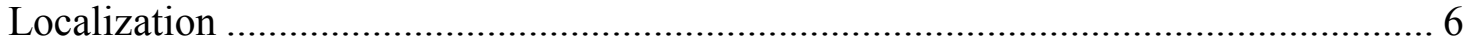

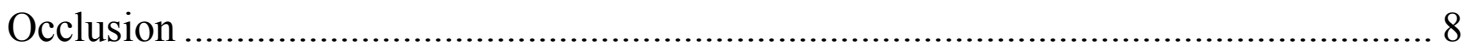

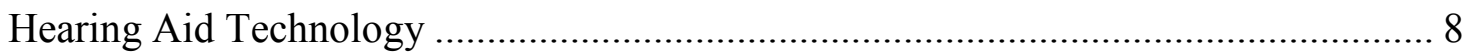

CHAPTER 3. THEORETICAL FRAMEWORK ....................................................... 11

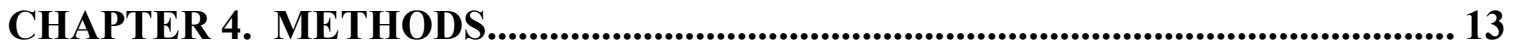

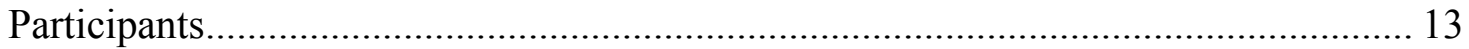

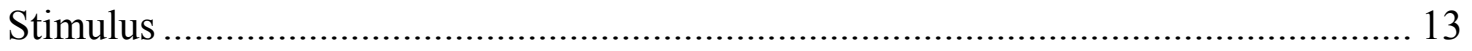

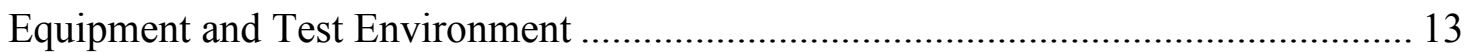

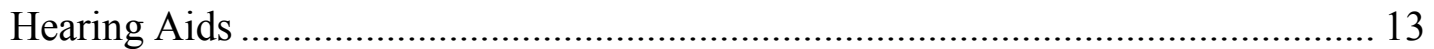

Localization Test Equipment and Environment .................................................. 19

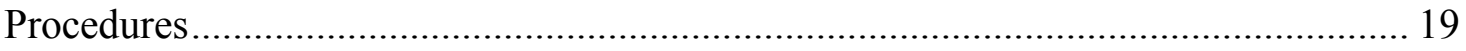

CHAPTER 5. RESULTS.................................................................................... 26

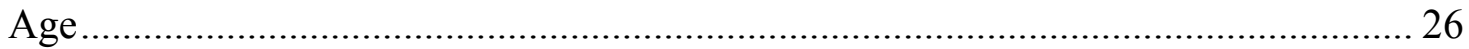

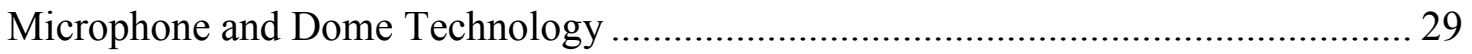

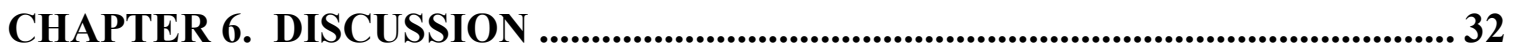

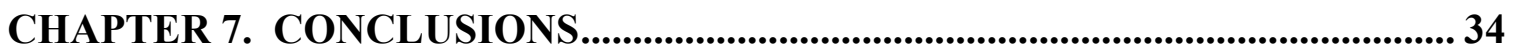

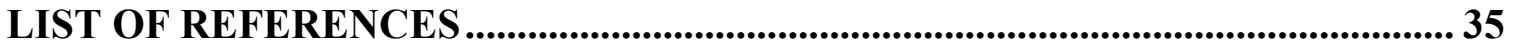

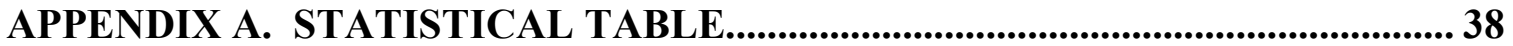

APPENDIX B. FIGURES AND GRAPHS ............................................................ 40

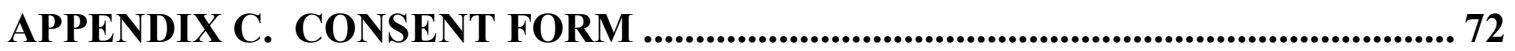

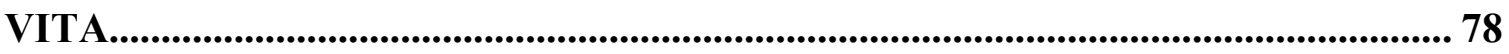




\section{LIST OF TABLES}

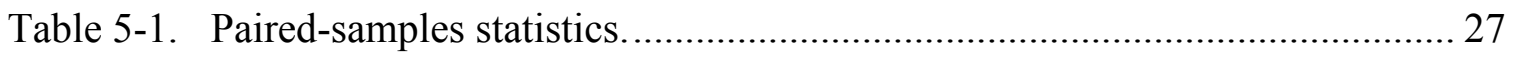

Table 5-2. Paired-samples t-test results for RMS error between participant groups..... 27

Table 5-3. Correlation of age and RMS error across all participant groups.................. 28

Table 5-4. Correlation of age and RMS error between $>50$ years and hearing

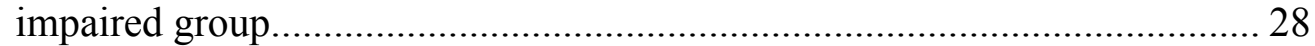

Table 5-5. Means and standard deviations for RMS error............................................ 30

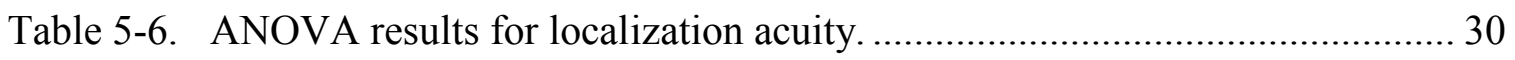

Table 5-7. One-way within-subject ANOVA results for dome................................... 31

Table A-1. RMS error values for each participant across each condition. ..................... 39 


\section{LIST OF FIGURES}

Figure 4-1. Young normal hearing participant mean hearing thresholds and standard deviations for left and right ears.

Figure 4-2. $\quad>50$ Normal hearing participant mean hearing thresholds and standard deviations for left and right ears

Figure 4-3. Hearing impaired participant mean hearing thresholds and standard deviations for left and right ears .............................................................. 16

Figure 4-4. Frequency analysis of "BASEBALL".................................................. 17

Figure 4-5. Picture of open-fit hearing instrument coupled to the ear.......................... 18

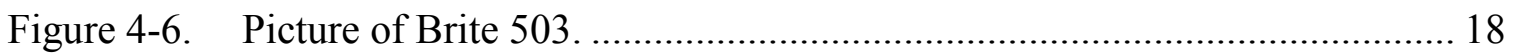

Figure 4-7. Picture micromold and open dome....................................................... 20

Figure 4-8. Picture of domes shown for size comparison to a dime............................ 20

Figure 4-9. Mean probe microphone measures averaged across ears for each dome condition (input signal level $=55 \mathrm{~dB}$ SPL).

Figure 4-10. Mean probe microphone measures averaged across ears for each dome condition (input signal level $=65 \mathrm{~dB}$ SPL).

Figure 4-11. Mean probe microphone measures averaged across ears for each dome condition (input signal level = $75 \mathrm{~dB}$ SPL) ............................................. 23

Figure 4-12. Diagram of test environment with speaker array.

Figure B-1. Young Normal Hearing Participants (A-F) perceived angle plotted as a function of actual target angle of the stimuli.

Figure B-2. Young Normal Hearing Participants (G-K) perceived angle plotted as a function of actual target angle of the stimuli..

Figure B-3. $>50$ Normal Hearing Participants (A-F) perceived angle plotted as a function of actual target angle of the stimuli.

Figure B-4. $\quad>50$ Normal Hearing Participants (G-J) perceived angle plotted as a function of actual target angle of the stimuli.

Figure B-5. Hearing Impaired Participants (A-F) unaided perceived angle plotted as a function of actual target angle of the stimuli.

Figure B-6. Hearing Impaired Participants (G-I) unaided perceived angle plotted as a function of actual target angle of the stimuli.

Figure B-7. Unaided best and worst perceived angle across each participant group plotted as a function of actual target angle of the stimuli.

Figure B-8. Hearing Impaired Participants (A-F) Open-Omnidirectional perceived angle across each participant group plotted as a function of actual target angle of the stimuli. 
Figure B-9. Hearing Impaired Participants (G-I) Open-Omnidirectional perceived angle across each participant group plotted as a function of actual target angle of the stimuli.

Figure B-10. Hearing Impaired Participants (A-F) Open-Adaptive Directional perceived angle across each participant group plotted as a function of actual target angle of the stimuli....

Figure B-11. Hearing Impaired Participants (G-I) Open-Adaptive Directional perceived angle across each participant group plotted as a function of actual target angle of the stimuli.

Figure B-12. Hearing Impaired Participants (A-F) Open-Fixed Directional perceived angle across each participant group plotted as a function of actual target angle of the stimuli..

Figure B-13. Hearing Impaired Participants (G-I) Open-Fixed Directional perceived angle across each participant group plotted as a function of actual target angle of the stimuli..

Figure B-14. Hearing Impaired Participants (A-F) Micromold-Omnidirectional perceived angle across each participant group plotted as a function of actual target angle of the stimuli.

Figure B-15. Hearing Impaired Participants (G-I) Micromold-Omnidirectional perceived angle across each participant group plotted as a function of actual target angle of the stimuli....

Figure B-16. Hearing Impaired Participants (A-F) Micromold-Adaptive Directional perceived angle across each participant group plotted as a function of actual target angle of the stimuli....

Figure B-17. Hearing Impaired Participants (G-I) Micromold-Adaptive Directional perceived angle across each participant group plotted as a function of actual target angle of the stimuli....

Figure B-18. Hearing Impaired Participants (A-F) Micromold-Fixed Directional perceived angle across each participant group plotted as a function of actual target angle of the stimuli.

Figure B-19. Hearing Impaired Participants (G-I) Micromold-Fixed Directional perceived angle across each participant group plotted as a function of actual target angle of the stimuli.....

Figure B-20. Hearing Impaired Subject A perceived angle across each hearing aid condition plotted as a function of actual target angle of the stimuli.

Figure B-21. Hearing Impaired Subject B perceived angle across each hearing aid condition plotted as a function of actual target angle of the stimuli....

Figure B-22. Hearing Impaired Subject $C$ perceived angle across each hearing aid condition plotted as a function of actual target angle of the stimuli. 
Figure B-23. Hearing Impaired Subject D perceived angle across each hearing aid condition plotted as a function of actual target angle of the stimuli.

Figure B-24. Hearing Impaired Subject E perceived angle across each hearing aid condition plotted as a function of actual target angle of the stimuli.

Figure B-25. Hearing Impaired Subject F perceived angle across each hearing aid condition plotted as a function of actual target angle of the stimuli....

Figure B-26. Hearing Impaired Subject $G$ perceived angle across each hearing aid condition plotted as a function of actual target angle of the stimuli.

Figure B-27. Hearing Impaired Subject H perceived angle across each hearing aid condition plotted as a function of actual target angle of the stimuli.

Figure B-28. Hearing Impaired Subject I perceived angle across each hearing aid condition plotted as a function of actual target angle of the stimuli.

Figure B-29. Scatterplot displaying correlation between participant age and RMS error.

Figure B-30. Unaided/Unamplified performance by Group..................................... 70

Figure B-31. Localization Acuity by Microphone Configuration within each Dome Condition. 


\section{CHAPTER 1. INTRODUCTION}

It is estimated that 31.5 million people in the United States are hearing impaired (Kochkin, 2005). Currently, there is no corrective treatment for the majority of those affected. Hearing aids provide the most viable treatment option for most individuals with hearing impairment. Of the individuals with a hearing loss, approximately $20 \%$ own hearing aids. Of the $20 \%$, approximately $30 \%$ are dissatisfied, while approximately $17 \%$ never use their hearing aids (Kochkin, 2005).

Many objective and subjective outcome measures are conducted clinically to verify hearing aid benefit; however, many individuals become dissatisfied with their hearing aids. Common complaints include: trouble hearing in background noise; poor sound quality; poor speech clarity; and unnatural sound quality (Souza \& Turner, 1994). The ability to process sounds and understand speech in complex acoustic scenarios is largely attributed to the binaural auditory system (Bronkhorst and Plomp, 1989). Another binaural process of the auditory system is azimuthal sound source localization. Although localization has not been used as a measure of subjective benefit in hearing aid outcome measures, one could speculate that disrupted spatial hearing would seem unnatural to the listener and thus lead to dissatisfaction with amplification.

Open-fit domes and micromolds represent new technologies in ear mold design that are considered ideal for hearing aids users with high-frequency, sensorineural hearing loss. The effect of these technologies might have on localization ability is unknown. Nonetheless, patients are routinely fit with hearing aids that have both adaptive technology (known to degrade performance) (Van den Bogaert et al., 2006) and open fitting ear molds. It is hypothesized that these two types of technologies may combine synergistically to potentially enhance or degrade localization acuity. Understanding how these technologies interact can help audiologists fit hearing aids to maximize binaural benefit for hearing aid users.

To date, research has examined how various hearing aid factors and occlusion affect localization acuity independently. Little is known about how these two technologies interact to affect localization despite the face that most people are fit with both technologies simultaneously.

\section{Localization Ability}

The ability to localize a sound source is a valuable asset in human audition. Accurate sound localization in the horizontal plane (azimuth) depends primarily upon the integration of interaural disparities in sound level and arrival time. Since the human auditory system is exquisitely sensitive to interaural level and time disparities, error in sound location in the azimuthal plane is very low with observed mean absolute error in adult listeners for broadband stimuli ranging from $\sim 2^{\circ}$ to $9^{\circ}$ azimuth (Markous and 
Middlebrooks, 1990; Bronkhorst, 1995), making sound localization a potentially robust and reliable assessment of binaural performance.

The ability to localize a sound source is dependent upon interaural time and level differences. Interaural time differences provide information for frequencies below 1500 $\mathrm{Hz}$, while interaural level differences provide information for frequencies above $1500 \mathrm{~Hz}$ (Gelfand, 2004). Thus, an individual with normal low frequency sloping to highfrequency hearing loss may have difficulty discriminating differences in level (volume) of a sound source and may become more reliant on interaural time discrepancies. Whereas an individual with impaired low-frequency rising to normal high-frequency hearing may have difficulty discriminating time differences, but may rely on level differences when localizing a sound source. Clinically, understanding this process may be essential when fitting amplification technology.

Impaired hearing degrades an individual's ability to localize sounds (Hausler, Colburn, \& Marr, 1983) and hearing aids often erode performance further. The effects of bilateral hearing aid use have been examined in two main capacities; degree of occlusion and hearing aid technology (Byrne et al., 1998; Noble et al., 1998; Drennan et al., 2005; Keidser et al., 2006; Van den Bogaert et al., 2006; Chung et al., 2008). Extensive research has been done on sound source localization within these areas. Open venting (degree of occlusion) preserves the impaired auditory system's ability to localize relative to the impaired unaided (without hearing aid) condition; however, open venting does not improve localization ability with these listeners (Nobel et al., 1998). Hearing aid technologies such as directional microphones and digital noise reduction have been shown to degrade a listener's ability to correctly localize a sound source relative to a listener's ability without a hearing aid (Hausler et al., 1983; Van den Bogaert, 2006). Most research involving amplification technology and venting has been conducted independently, therefore the effect of combined technology is unknown.

\section{Occlusion}

The effect of ear mold venting on localization ability has been examined (Byrne et al., 1998; Noble et al., 1998). Localization acuity is better with open, less occluding, ear molds that allow the listener to utilize the direct sound field for localization of sound on the horizontal and vertical planes independent of hearing loss configuration (Noble et al., 1998). However, aided performance is not better than unaided performance. Consequently, these results suggest that open ear molds may preserve localization ability of the listener, but do not improve localization ability deficits caused by hearing loss.

Open-fit, behind-the-ear (BTE) hearing aids have a variety of fitting options appropriate for varying degrees of hearing loss. Open domes allow the ear canal to be un-occluded and are appropriate for normal to mild low frequency sloping to a moderate high frequency hearing loss. Micromolds, which can also be referred to as custom tips, are more occluding and are appropriate for normal to mild low frequency sloping to moderately severe high frequency hearing losses. Micromolds provide a greater degree 
of occlusion to lessen the chances of feedback in individuals with a greater severity of hearing loss. That stated, many patients have hearing configurations that border both fitting guidelines. Therefore, it is imperative that the clinician understand the effect of both fitting options to enhance patient satisfaction.

\section{Hearing Aid Technology}

\section{Microphone Placement}

Noble and Byrne (1990) investigated localization ability within the vertical and horizontal planes based on microphone placement. Three styles of hearing instruments were examined: behind-the-ear (BTE) with microphones at ear level above the pinna; inthe-ear (ITE) with microphones in the concha; and in-the-canal (ITC) with microphones in the opening of the ear canal. Listeners were tested in omnidirectional microphone configurations. No significant difference was noted between unaided and aided performance across hearing aids. Thus, the findings of this study reported that localization ability did not improve with BTE, ITE, or ITC hearing instruments in omnidirectional microphone configurations regardless of microphone location.

\section{Directional Technology}

Van den Bogaert et al. (2006) examined the effects of bilateral sensorineural hearing loss and bilateral adaptive directional microphone technology on horizontal localization acuity. Results indicated that bilateral hearing aid users performed more poorly than normal hearing listeners, and that bilateral adaptive microphone directionality degraded localization cues relative to unaided hearing.

While the aforementioned study (Van den Bogaert et al., 2006) provides insight regarding the use of bilateral adaptive hearing aid technology, several limitations of this study should be noted. First, the experimental group (older adults) was not age matched to the normal hearing control group (young adults). It has been suggested that due to decreased mylenation and neural synchrony after age 45 , it can be assumed that binaural integration and temporal processing of an older adult differs from a younger adult and has been shown in the P300 response (Polich, 2004). Thus, localization performance may differ from a normal hearing older adult when compared to a normal hearing young adult as the ability to localize a sound source is derived from the ability to discriminate between interaural time and level differences. Second, the hearing aid manufacturer was not controlled for within this study. One must assume that the adaptive directional cardioids plots and compression onset algorithms could differ between manufacturers and increase performance variability. Finally, each participant used their own personal ear mold and therefore venting size varied between participants. Ear mold venting is known to affect localization ability. Consequently, a systematic, well-controlled evaluation of 
the effects of combined effects of occlusion and directional technology on localization ability is needed.

\section{Rationale}

Although the aforementioned studies have provided valuable information regarding degree of occlusion and hearing aid factors independently, the combined effects of the two are unknown. The overall objective of this study is to investigate how horizontal localization acuity may be affected by the combined use of open-fit ear mold design and directional microphone technology when level cues are minimized. Prior work has noted that adaptive directionality can have a negative impact on horizontal localization performance and binaural cues (Van den Bogaert et al., 2006). Consequently, it has been hypothesized that the degradation in performance seen with adaptive hearing aid technologies may be caused by a disruption in interaural temporal cues.

Open-fit domes and micromolds represent new technologies in ear mold design and are considered ideal for hearing aid users with high-frequency, sensorineural hearing loss. Each dome type provides less occlusion than traditional earmolds as neither are 'custom' products. Patients utilizing this type of 'open-fitting' typically have normal to mild low frequency hearing with varying degrees of high frequency hearing loss. Depending on the anatomy (shape) of an individual's ear canal and configuration of hearing loss, either dome may be employed by an audiologist.

Although it may be speculated, the effect that these technologies (microphone and dome) might have on localization acuity is unknown as open-fit amplification technology has yet to be evaluated. Nonetheless, patients are routinely fit with hearing aids that have both adaptive technology (known to degrade performance) and open fitting ear molds. It is hypothesized that these two types of technologies may combine synergistically to potentially enhance or degrade localization acuity. Understanding how these technologies interact can help audiologists fit hearing aids to maximize binaural benefit for hearing aid users.

Lastly, most hearing aid users are age 55 or older. Studies assessing localization acuity in geriatric populations are scarce. Prior work assessing localization ability in hearing impaired listeners has not evaluated the effect of age, as the mean age within control groups in previous research is considerably less than the mean age of investigational groups. Furthermore, previous research has investigated microphone technology and occlusion separately, but not the interaction of both technologies within a given listener. In addition, prior research investigating the effects of microphone technology on localization acuity has not controlled for hearing aid manufacturer; which could allow for variance in performance between participants. Finally, open-fit amplification employs various occlusion and microphone technologies providing diverse fitting capabilities to hearing impaired listeners; however, it is unclear how the combination of said technologies affect the horizontal localization ability of a given listener. Prior work evaluating receiver placement in open-fit technology demonstrated 
that receiver placement does not affect objective performance within a listener (Alworth et al., 2010). Thus, this study aims to determine the effect of high-frequency, sensorineural hearing loss on azimuthal sound localization acuity in the horizontal plane within geriatric listeners utilizing open-fit hearing aid technology.

The specific research questions of this study were as follows:

1. Did age affect azimuthal localization acuity in adult listeners?

2. Did microphone technology (omnidirectional, adaptive directional, fixed directional) in an open-fit hearing aid affect azimuthal localization acuity within a geriatric listener with high-frequency, sensorineural hearing loss when type of dome (occlusion) is controlled?

3. Did dome type (open vs. micromold) within an open-fit hearing aid affect azimuthal localization acuity within a geriatric listener with high-frequency, sensorineural hearing loss when microphone type is controlled?

4. Was there a synergistic effect between new ear mold designs (open-fit hearing aid dome and micromolds) and microphone programs (adaptive-directional, omnidirectional, and fixed-directional) on azimuthal localization acuity in geriatric listeners with high-frequency, sensorineural hearing loss? 


\section{CHAPTER 2. REVIEW OF LITERATURE}

\section{Localization}

Binaural sound localization was first described as a combination of two processes and termed the Duplex Theory by Lord Rayleigh in the early 1900s (as cited in Gelfand, 2004). The Duplex Theory explains binaural localization based upon time differences at lower frequencies, and level differences at higher frequencies between ears. Gelfand (2004) further explains this process by elaborating on how such differences are established. Low frequency information contains longer wavelengths, which at times may be longer than the path around the head. Simply stated, if a low frequency sound is presented to one side of the head and the wavelengths are longer than the path around the head, the sound waves will 'bend' around the head to the far ear. Thus, interaural time differences (ITD) provide localization cues for lower frequency information. Conversely, interaural level differences (ILD) provide localization cues for high frequency information. High frequency information contains smaller wavelengths. Shorter wavelengths are smaller than the path around the head, and are thereby blocked in reaching the far ear. Consequently, when a signal is blocked from reaching the far ear the signal will seem louder/more intense in the ear nearer the signal.

Hausler and colleagues (1983) further investigated sound localization ability in adult listeners by evaluating varying degrees of hearing impairment. One hundred forty listeners participated in several localization measurements. Measurements were obtained in the soundfield and under headphones. Measurements in the soundfield obtained information with regard to minimal audible angle (MAA) while measurements under headphones provided information regarding the just noticeable difference (JND). Stimuli for both soundfield and headphone conditions included a broadband $(.25-10 \mathrm{kHz})$ pulsed noise presented at suprathreshold levels. Overall, results of this study noted characteristic impairments of sound localization in different types of hearing loss. Characteristic impairments of listeners with: conductive hearing loss were interpreted as having bone conduction effects; sensorineural hearing loss were interpreted as consequences of impaired or preserved spectral processing; neuromas were interpreted as having signal transmission effects within the auditory nerve; and those with central involvements were interpreted as having separate processes that exist at some level for different localization cues. Taken together, the authors concluded that their results suggest sound localization tests may provide more insight to audiological function than previously thought. Thereby suggesting that sound localization testing might assume a greater importance in otoneurological exams in the future.

Bronkhorst and Plomp (1989) evaluated binaural speech intelligibility in noise for adult hearing impaired listeners. The effects of head-induced interaural time and level differences were evaluated within groups of symmetrical, asymmetrical and normal hearing listeners. Participants' speech recognition thresholds for sentences presented with noise were determined as a function of noise azimuth, binaural cue, and interaural difference in presentation level. Results indicated that symmetrical bilateral hearing 
impaired listeners benefited less from interaural level differences when compared to their normal hearing counterparts. However, when stimulus presentation level is equal, symmetrical bilateral hearing impaired listeners do not differ significantly in performance compared to normal hearing listeners. Analysis of asymmetrical bilateral hearing impaired listeners yielded different results. When compared to normal hearing listeners, asymmetrical bilateral hearing impaired listeners perform worse in all conditions (level and time). Thus, the authors concluded, in general, that adult hearing impaired listeners benefit less from interaural level differences than listeners with normal hearing. It was also noted that hearing impaired listeners and normal hearing listeners benefit from interaural time differences almost equally.

Makous and Middlebrooks (1990) investigated the ability of adult listeners with two-dimensional sound localization. More specifically, the authors assessed the utility of the head pointing response in sound localization studies and the spatial dependence of localization performance. All participants were normal hearing and participated in both open and closed loop localization tasks. Open loop tasks involve brief stimulus presentation; whereas closed loop tasks entail continuous stimulus presentation. Within localization tasks, both sensory and response components were employed. First, the participant determined the location of the stimulus, then reported the location by orienting to it. Results indicated that at most stimulus locations, participants performed better for closed loop trials than open loop trials. Further, sound localization performance in front of the participant displayed a decrease in performance with increasing stimulus azimuth. This decrease in performance is generally consistent with the spatial dependence of interaural level differences. Stated differently, results of this study indicated that the rate of change of interaural level differences decrease with increasing azimuth, and the just noticeable difference tends to increase with increasing interaural level differences.

In 1995, Bronkhorst evaluated localization ability of real and virtual sound sources in adult listeners. Eight individuals with normal hearing participated. Virtual sound sources were generated using head-related transfer functions that were measured utilizing probe microphone measurements. Listeners participated in two tasks. In the first task, listeners turned their head until they perceived they faced a sound source that was continuously on. In the second task, the stimulus was a short (non-continual) sound, and listeners indicated what quadrant the source was located and if it was above or below the horizontal plane. Stimuli included harmonic signals with a fundamental frequency of $250 \mathrm{~Hz}$, and an upper frequency ranging from 4000 to $15,000 \mathrm{~Hz}$. Results of this study indicated that listeners performance was similar for real and virtual sources for each task, provided the stimuli did not contain frequencies above $7000 \mathrm{~Hz}$. When frequencies up to $15,000 \mathrm{~Hz}$ were included, listener performance on virtual sources was poorer than real sources. The authors noted that differences were likely attributed to the distortion of high frequency spectral cues in the head related transfer functions introduced by the probe microphone measurements within the virtual sound source condition. 


\section{Occlusion}

Noble et al. (1998) investigated the effect of open earmolds on localization ability in adult listeners with impaired hearing. All participants were experienced hearing aid wearers (experienced with bilateral, behind-the-ear hearing aids coupled with closed earmolds) with normal to slight hearing loss in the low frequencies sloping to moderate to severe hearing loss in the high frequencies. Testing evaluated participant performance on localization tasks in unaided (without hearing aid) and aided conditions with their personal hearing aids coupled to closed, vented, and open earmolds. As microphone technology was not specifically evaluated within this study, all participants used their personal hearing aid settings throughout testing. Results of this study revealed that closed earmolds degraded localization performance relative to unaided (without hearing aid); however, vented and open earmolds restored localization performance to unaided (without hearing aid).

In 1998 Byrne and colleagues evaluated open earmold fittings with regard to aided sound source localization ability for adult listeners with moderate low frequency rising to slight/normal high frequency hearing sensitivity. Twenty- two listeners participated. Localization tasks were completed in the horizontal and vertical plane within four conditions (without hearing aid, aided with closed earmold, aided with open earmold, aided with sleeve earmold). Results of this study indicated that localization within the vertical plane was significantly poorer for the closed earmold condition relative to unaided (without hearing aid) and the sleeve earmold condition was nearly equal to unaided performance within the vertical plane. Benefit of open earmolds when compared to closed earmolds was related to hearing level. Listeners with the best high frequency hearing received the most benefit. No significant findings were noted within the horizontal plane. Thus, the authors concluded that non-occluding (open) earmolds optimize aided vertical localization for hearing aid users with good high frequency hearing.

\section{Hearing Aid Technology}

Noble and Byrne (1990) compared binaural hearing aid systems with regard to localization in the horizontal and vertical planes. Participants included three groups of adult hearing-impaired listeners who were fit with behind-the-ear (BTE), in-the-ear (ITE), or in-the-canal (ITC) hearing aids. Spatial localization was tested within the horizontal and vertical planes. ITC wearers displayed a deterioration in aided performance when compared to unaided. Specific reasons for the decrement noted with ITC wearers could not be identified, although it was noted that it may have been due to their own personal systems. However, no significant differences in unaided (without hearing aid) performance between groups was observed, nor between unaided and aided performance for the BTE and ITE groups.

In 2005, Drennan and colleagues assessed the ability of adult hearing impaired listeners to localize and identify speech in noise when using phase preserving, and non- 
phase preserving hearing aid technology. Participants within this study were fit bilaterally utilizing the National Acoustic Laboratory linear frequency-gain characteristic with the hearing aids programmed in one of two ways: 1) included a linear-phase filter, 2) included filters designed to compensate for the magnitude and phase peaks caused by the hearing aid fitting, thereby preserving interaural phase. Participants were asked to identify a word and its location while in the presence of background noise that contained a speech shaped spectrum. This task was performed a total of 4 times over the course of each 16-week periods while the participant was fit with the hearing aids. Results indicated that immediately after being fit with hearing aids, regardless of the program setting, that listeners' ability to localize speech in noise was reduced. That stated, the phase-preserving processing program had a less detrimental effect on localization ability immediately after fitting. After three weeks of wearing the hearing aids, performance improved and no detrimental effect of amplification was noted for localization ability in noise with either processing strategy. A difference in performance was not noted until later in the trial period. After 16 weeks, speech understanding for phase-preserving processing was slightly, but significantly better than linear phase processing. Thus, listeners have the ability to quickly acclimate to altered speech cues regardless of amplification strategy.

Keidser and colleagues (2006) evaluated the effect of multi-channel wide dynamic range compression, directional microphones, and noise reduction systems on horizontal localization ability relative to linear amplification on adults with impaired hearing. Twelve listeners participated and were bilaterally fit with behind-the-ear devices. Localization testing utilized broadband pulsed pink noise in a $360^{\circ}$ loudspeaker array. Testing was conducted two weeks and two months post-fitting. Of the three signal processing strategies, directional microphones had the most detrimental effect on horizontal localization performance over time.

In 2006, Van den Bogaert and colleagues investigated the effect of bilateral hearing aids on directional hearing within the frontal horizontal plane in adults with impaired hearing. More specifically, the following questions were addressed: How do adult bilateral hearing aid wearers perform on localization tasks when compared to their normal hearing counterparts? Do bilateral hearing aids preserve localization cues? How does adaptive directionality technology affect localization performance? Adult hearing aid wearers were tested with and without their hearing aids. When tested with their hearing aids, participants were tested in omnidirectional and adaptive directional microphone configurations. The normal hearing control group consisted of ten young adult participants that were not age matched to the hearing impaired participant group. Within the hearing impaired group, all were experienced bilateral hearing aid wearers over the age of 50. Brand, type, venting and degree of hearing loss were not controlled within the hearing impaired group; however, all amplification levels for hearing aid wearers did not display significant asymmetry. Results of this study revealed that bilateral adaptive directional hearing aid wearers perform more poorly than normal hearing participants in horizontal localization tasks. Approximately one half the hearing impaired participants reached normal performance when tested unaided. Bilateral 
hearing aids did not preserve localization cues within this study. Further, adaptive directionality significantly degraded horizontal localization performance.

Chung et al. (2008) evaluated the effects of in-the-ear (ITE) microphone directionality on localization. Two groups of adult participants were included; experienced hearing aid wearers and normal hearing listeners. Participant groups were not age matched. Hearing impaired participants were first fit with custom ITE linear hearing aids containing microphones with adjustable directionality. Four microphone configurations were evaluated: omnidirectional, cardioid, hypercardioid, and supercardioid. All microphone conditions were evaluated within both quiet and noisy localization conditions. Results indicated that the young normal hearing adult participants performed better on sound localization than older adult participants with hearing loss. It should be noted that since the participant groups were not age matched, it is unclear whether this difference can be attributed to hearing loss, age, or both. Further, directional microphones (cardioid, hypercardioid, supercardioid) did not degrade localization performance in hearing impaired participants relative unaided (without hearing aid). These results, therefore suggest that the use of directional microphones may be considered as method to maintain sound localization ability at least when utilizing linear hearing aids with fixed directional patterns.

In 2010, Alworth and colleagues evaluated the effects of receiver placement on subjective and objective measures in open-fit hearing instruments. Participants included both novice and experienced hearing aid wearers. Each participant trialed each type (receiver-in-the-ear, receiver-in-the-aid) of hearing instrument for 6 weeks and reported their satisfaction with each instrument across several subjective categories. Results of this study indicated that receiver-in-the-ear instruments had significantly greater maximum gain before feedback (specifically at 4000 and $6000 \mathrm{~Hz}$ ), greater user satisfaction ratings and overall preference. However, all other objective measures were not significantly different between the two instruments. This study further highlights the clinical importance of obtaining subjective measures as a way to measure aided benefit; as all participants performed equally well on objective measures with each type of openfit instrument. 


\section{CHAPTER 3. THEORETICAL FRAMEWORK}

Theoretically, a listener's ability to localize a sound source can be based upon the Duplex Theory. The Duplex Theory simply states that sound source localization is a binaural process involving interaural timing and level cues (Lord Rayleigh, 1907 as cited in Gelfand, 2004). Interaural level differences discriminate high frequency information between ears, while interaural time differences discriminate low frequency information between ears (Gelfand, 2004). Given this, one may assume (as research has shown) that individuals with hearing loss will have marked difficulty localizing a sound source when compared to normal hearing individuals.

Individuals with a hearing loss have difficulty localizing a sound source due to the inability to use ILD and ITD information efficiently due to inaudibility of some sounds. Hearing aids amplify inaudible sounds, but such things as microphone technology, placement, and degree of occlusion all hinder localization performance. Degree of occlusion has been proven to degrade localization performance relative to unaided (without amplification). The less occluded an individual's ear canal, the more likely they are able to localize relative to their unaided performance as they have access to natural ITD cues. Stated differently, a listener has access to more low frequency information with lesser amounts of occlusion; thereby granting the listener access to natural ITDs.

Adaptive, fixed directional and omnidirectional microphone configurations have been shown to degrade adult listeners' ability to localize a sound source when compared to their unaided performance. However, amplification restores access to high frequency information to a listener with high frequency hearing loss. One would speculate that the preservation of ILDs (access to high frequency information) should aid in localization ability of hearing impaired listeners. However, previous research has consistently shown that hearing aids degrade a listener's ability to localize a sound source. That stated, prior work evaluating microphone technology within the area of horizontal localization has not controlled for manufacturer nor type of earmold which could potentially add variance between participants. Thus, the potential interaction of type of microphone technology and degree of occlusion is unknown. Ideally, it may be speculated that, for a given condition, amount of occlusion and type of microphone technology may work synergistically to lessen degrading effects on a listener's ability to localize within the horizontal plane. Taken together, it may be hypothesized that by preserving access to low frequency information (ITD) and restoring access to high frequency information (ILD) within a hearing impaired listener that unaided (without amplification) horizontal localization ability may equal that of their normal hearing counterparts.

Open-fit technology has yet to be evaluated within this area of research. Open-fit hearing aids can employ several microphone technologies and dome configurations for a given patient. Domes are considered less occluding than traditional earmolds, as they do not require a custom earmold impression. However, it is unknown if the "most occluding' dome would vastly different from the 'least occluding' dome with regard to horizontal localization ability within a given patient as both domes are considered 'open.' 
The effects of aging have not been evaluated extensively in prior horizontal localization research involving adult listeners. Most studies directly compare older hearing impaired adult listeners to groups of young normal hearing adult listeners with the mean age of each group separated by $40+$ years. Previous neurophysiological research has shown that age related factors can affect neural responses in older listeners. Therefore, it may be assumed that older normal hearing listeners will perform differently than young normal hearing listeners on horizontal localization tasks.

Taken together, the following hypotheses are stated:

1. Age will affect a listener's horizontal localization ability.

2. Omnidirectional, adaptive directional and fixed directional microphones will affect horizontal localization ability similarly.

3. Dome type will affect a listener's horizontal localization ability.

4. Open-fit technology (utilizing a combination of microphone and dome technology) will improve a hearing impaired listener's localization ability to 'normal.' 


\section{CHAPTER 4. METHODS}

\section{Participants}

Thirty adults participated in this experiment. Eleven young adults with normal hearing (mean age: 24 years; range: 5 years), ten older adults with normal hearing (mean age: 57 ; range: 15 years) and nine adults with hearing impairment (mean age: 75 years, range: 9 years) recruited from within the Knoxville, Tennessee community participated in this study. The participants with normal hearing [Note: Normal hearing was determined by obtaining audiometrically measured pure-tone air conduction thresholds of $25 \mathrm{~dB}$ HL or better (ANSI S3.6, 1996) at octave intervals between 250 and $4000 \mathrm{~Hz}$ ] participated in one, twenty minute experimental session. Participants with hearing impairment [Note: Hearing impaired participants all displayed a symmetrical highfrequency, sensorineural hearing loss characterized by normal/mild low frequency hearing loss $(250$ to $750 \mathrm{~Hz}$ ) sloping to a moderate/severe high frequency hearing loss $(2000$ to $8000 \mathrm{~Hz})$ which fit the guidelines of the hearing instruments utilized within the study; please refer to Figures 4-1 through 4-3 for hearing thresholds within each participant group] participated in two experimental sessions. It should be noted that all hearing impaired participants were successful experienced open-fit hearing aid wearers. Further, each participant had been fit with the manufacturer and type of hearing aid utilized within this study approximately one year prior to participation within this experiment.

All participants were required to sign an informed consent form before participating in the study. All qualification and experimental testing was conducted in a sound-treated examination room (IAC; $2.2 \times 1.8$ meters) with ambient noise levels suitable for testing ears uncovered (ANSI S3.6, 1991).

\section{Stimulus}

The spondee "baseball" was digitally recorded with a male voice at a sampling rate or $44 \mathrm{kHz}$, root mean squared (RMS) equalized, and stored as a wav file (see Figure 4-4). The level of the stimulus was calibrated to $60 \mathrm{~dB}$ SPL and was randomly varied on a trial-by-trial basis between 52 and $68 \mathrm{~dB}$ SPL (roved $\pm 8 \mathrm{~dB}$ ).

\section{Equipment and Test Environment}

\section{Hearing Aids}

Hearing impaired participants who met the aforementioned inclusion criteria were fit bilaterally with open-fit, digital behind-the-ear hearing instruments (model: BRITE 503, Bernafon; see Figures 4-5 and 4-6) with multi-channel wide dynamic range 


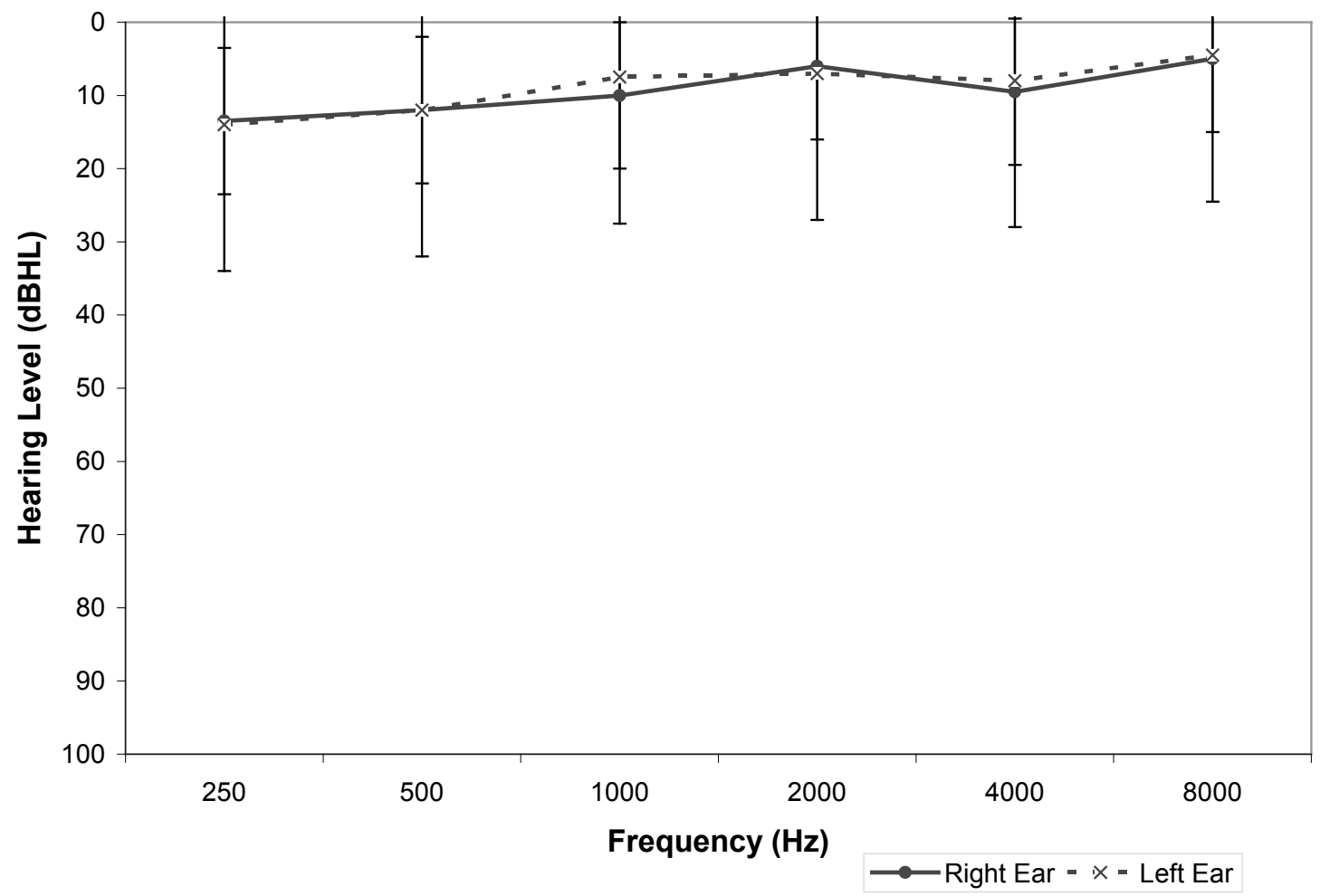

Figure 4-1. Young normal hearing participant mean hearing thresholds and standard deviations for left and right ears. 


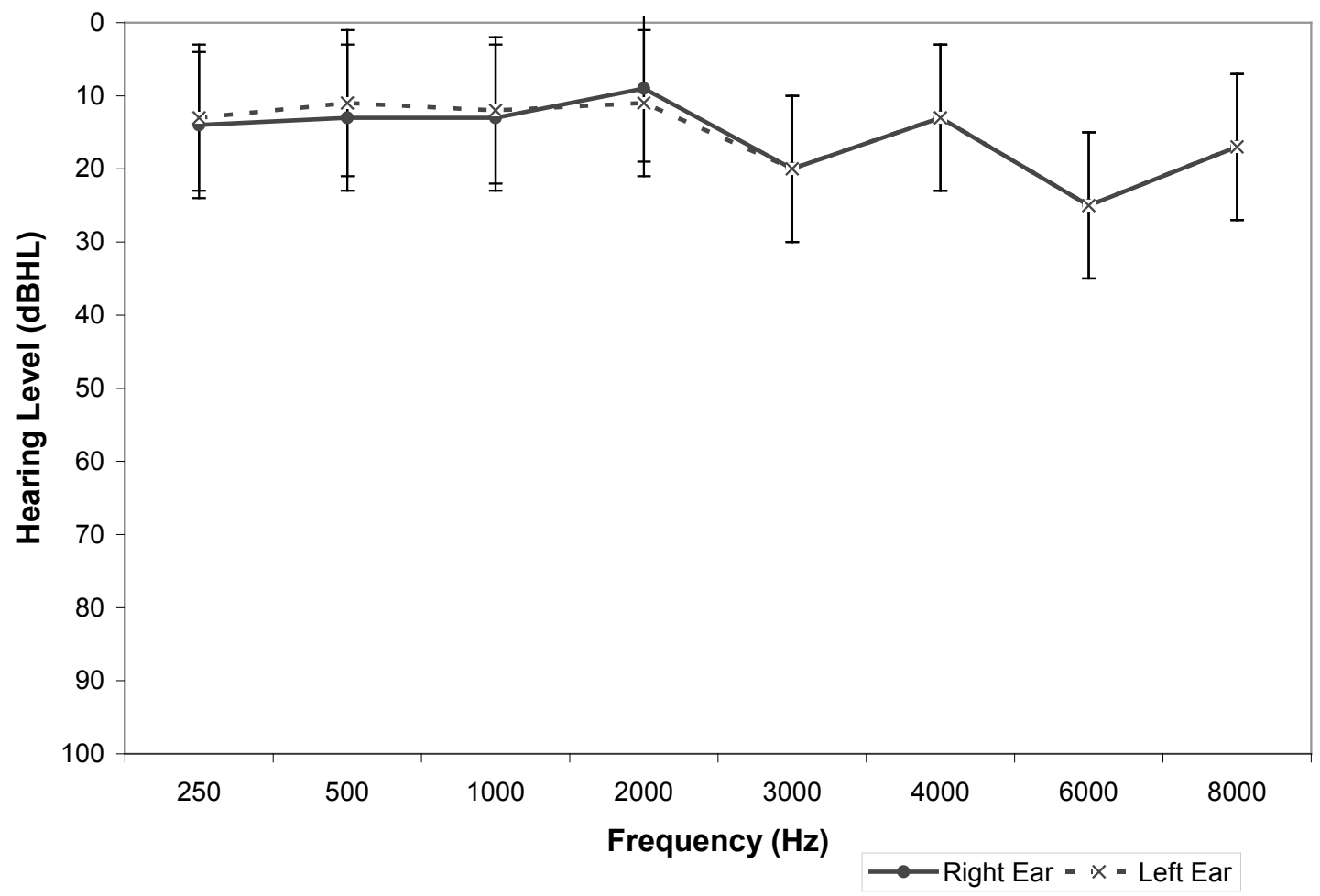

Figure 4-2. $>50$ Normal hearing participant mean hearing thresholds and standard deviations for left and right ears. 


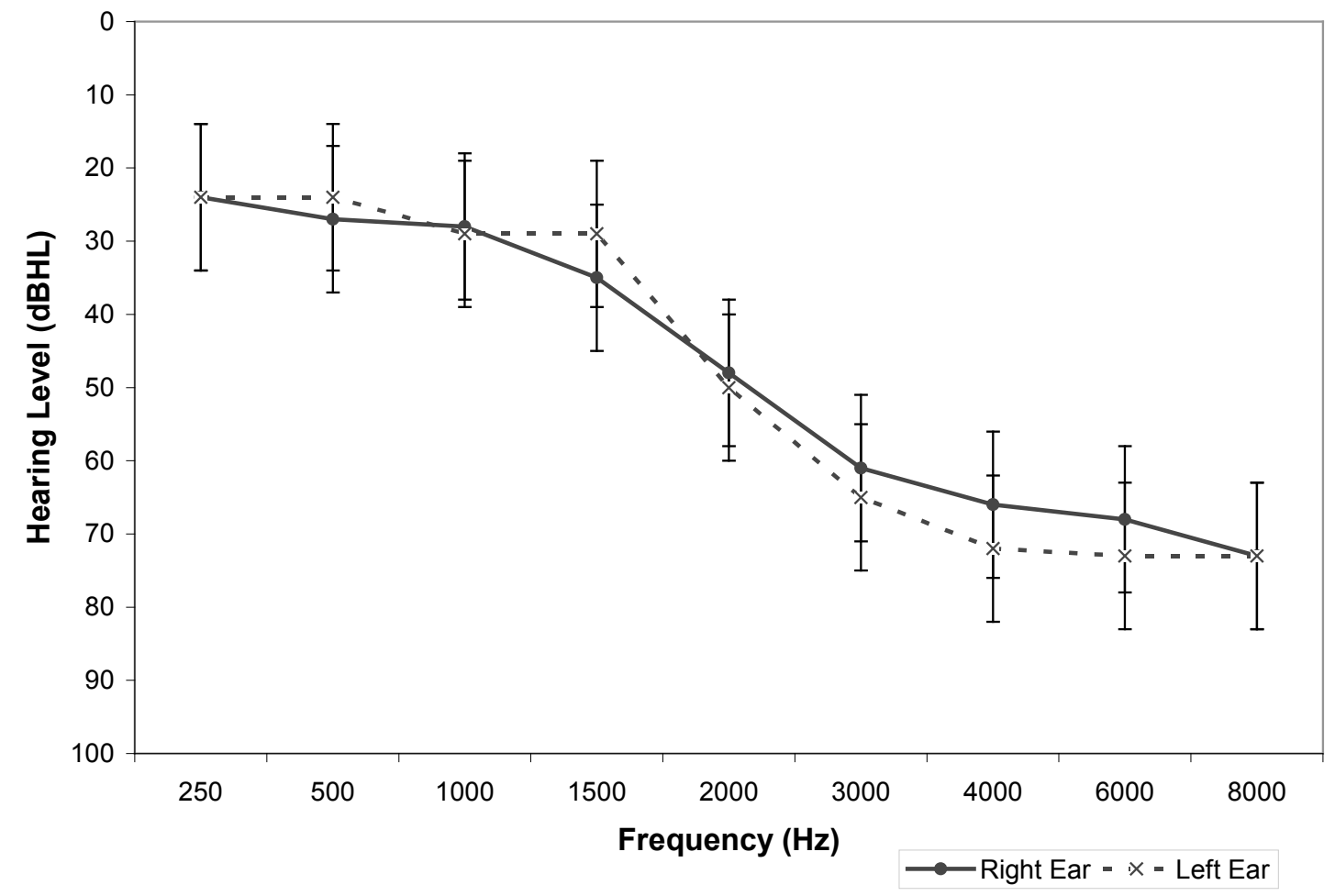

Figure 4-3. Hearing impaired participant mean hearing thresholds and standard deviations for left and right ears. 


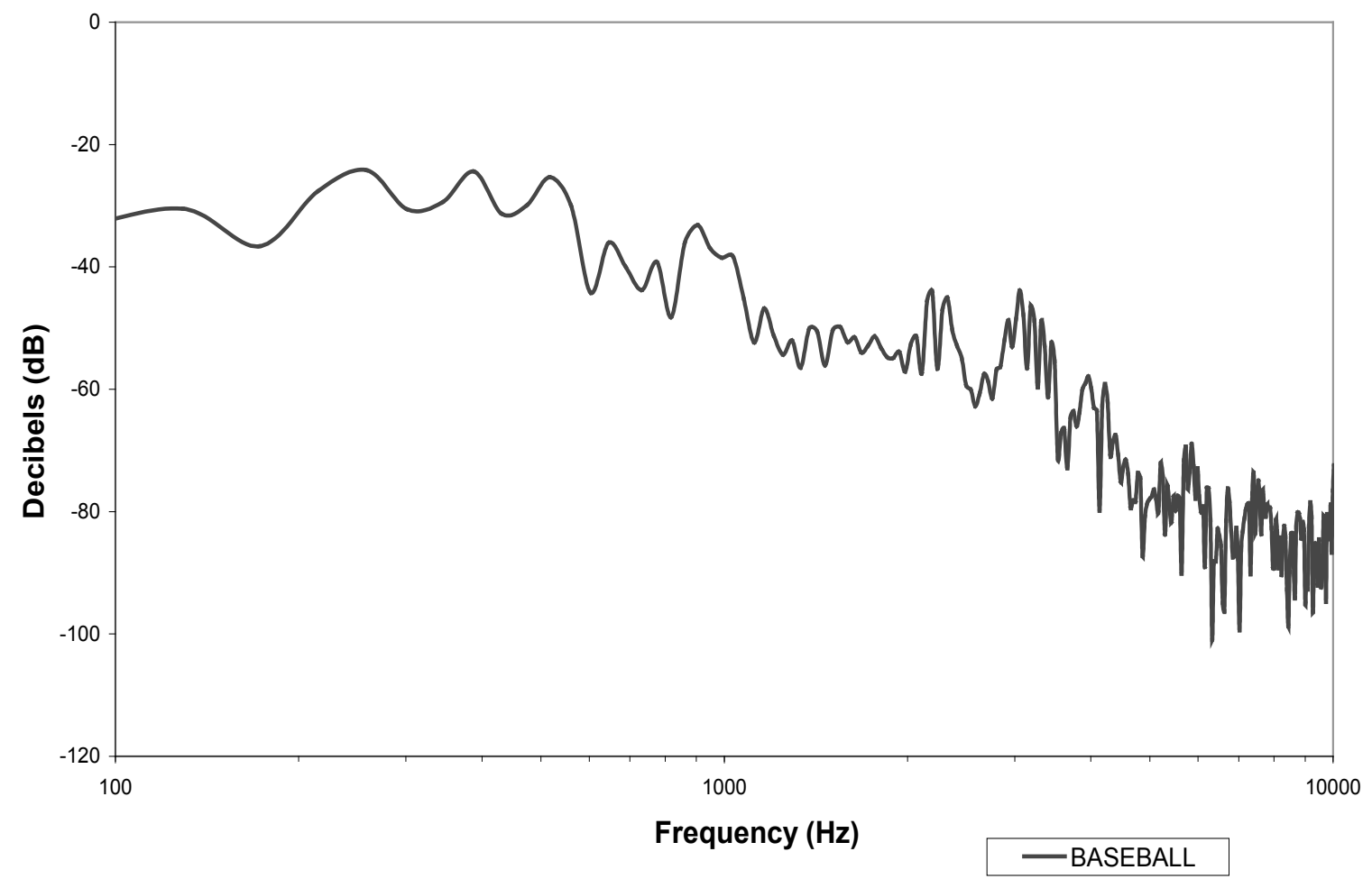

Figure 4-4. Frequency analysis of "BASEBALL." 


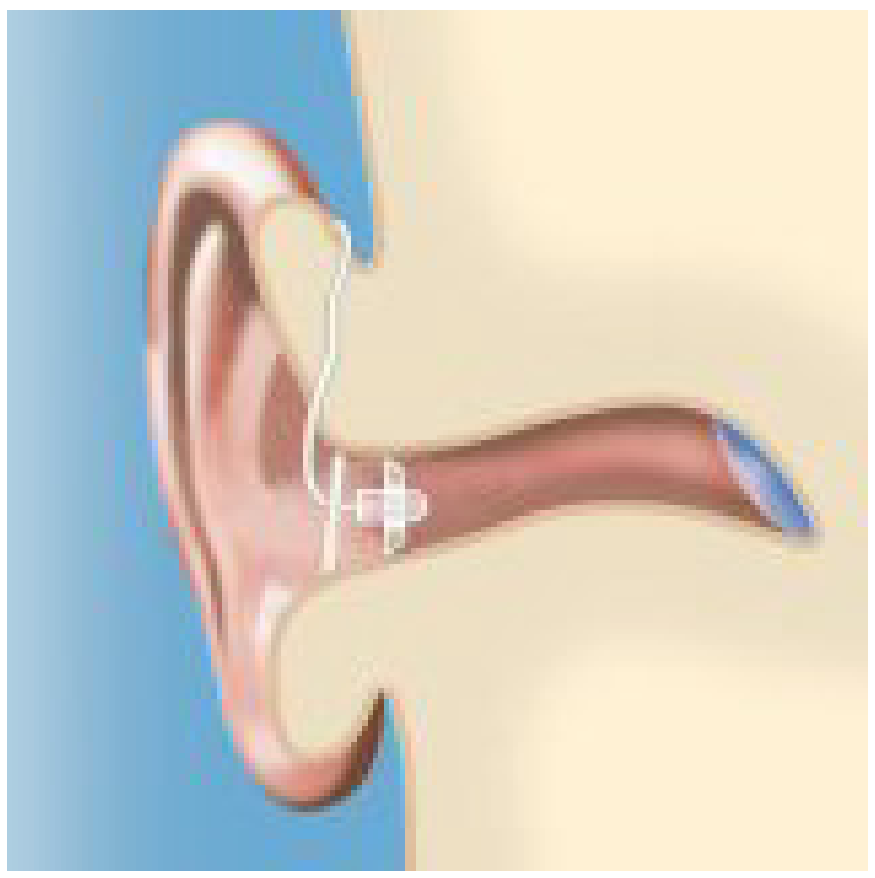

Figure 4-5. Picture of open-fit hearing instrument coupled to the ear.

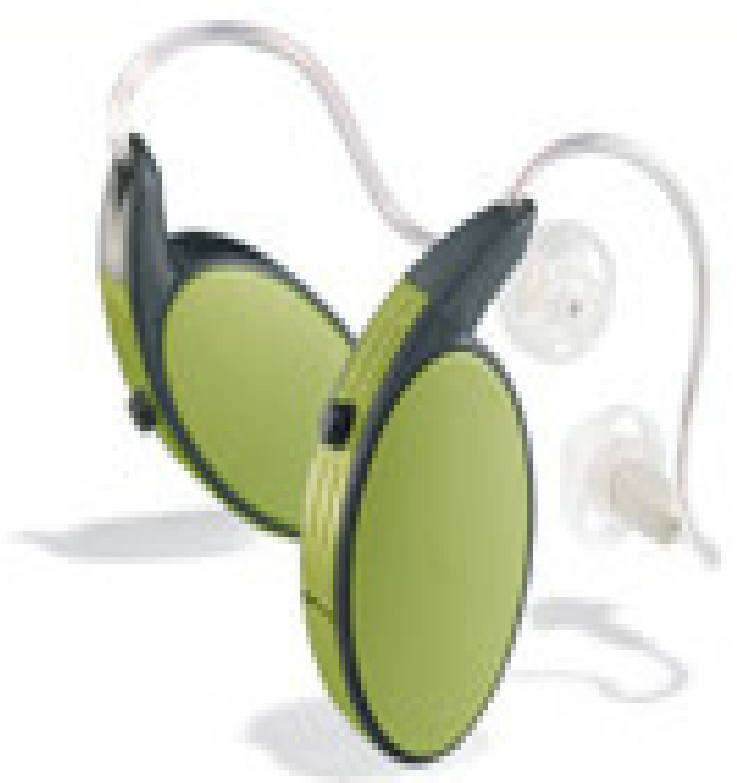

Figure 4-6. Picture of Brite 503. 
compression processing and multiple memory capabilities. The hearing aids utilized for this study were consignment aids provided by Bernafon for research purposes; therefore, the same two hearing aids were used for each participant. Each participant was fitted with an open dome and a micromold (see Figures 4-7 and 4-8). The hearing aids were initially programmed using the proprietary fitting algorithm software, Bernafon OASIS Plus version 8.5 (Bernafon Hearing Instruments, Inc) and the NAL-NL1 prescriptive approach. Probe microphone measures were conducted to verify match to NAL-NL1 targets for each participant for both ear mold conditions (open dome, micromold) using the Audioscan (Verifit) Verifit Open fittings with the Speechmap function at 55, 65, and 75 dB SPL (Figures 4-9 through 4-11). Three memories were programmed randomly for each participant with the following microphone settings: (i) adaptive directionality, (ii) fixed directionality, and (iii) omni-directionality for each dome condition. Other features (digital noise reduction and expansion) were deactivated during testing to ensure that each memory had identical fitting parameters. Prior to data collection, an experimental schedule was generated for each participant that listed a completely randomized assignment of memories with each dome condition. Dome conditions as well as an unamplified (baseline) were also randomized for each participant.

\section{Localization Test Equipment and Environment}

Testing was conducted in a sound- treated booth (IAC, $2.2 \times 1.8$ meters). Participants sat in a classroom (chair-style) desk facing a semicircular array of fifteen loudspeakers (Cambridge Sound Works Center/Surround IV; matched within $1 \mathrm{~dB}$ SPL at $100 \mathrm{~Hz}$ to $8000 \mathrm{~Hz}$ ) placed at $10^{\circ}$ intervals on an arc (with a radius of 1 meter) between $-70^{\circ}$ and $70^{\circ}$ azimuth (see Figure 4-12). A small picture was fastened below each loudspeaker. These pictures corresponded to an arc of pictures displayed on a computer screen during each trial. One trial consisted of a single presentation of the word "baseball."

Hardware including Tucker Davis Technologies (TDT) System III (RP2, PM2, AP2), in conjunction with an IBM PC host, controlled for stimulus presentation. This hardware also controlled the multiplexer used for loudspeaker switching and amplification. Software for the stimulus presentation and data collection operated on a custom written MatLab platform.

\section{Procedures}

The participants were seated in a classroom (chair-style) desk facing a loudspeaker at $0^{\circ}$ azimuth. A flat computer screen was located below the loudspeaker at $0^{\circ}$ azimuth, directly in front of the participant. A mouse and mouse pad were located on the desk. The participants were instructed to face forward and look at the computer screen. A single trial consisted of the presentation of the word "baseball." Each loudspeaker had a different small picture below it which corresponded with pictures on the computer screen. The participants were instructed to report where the word 


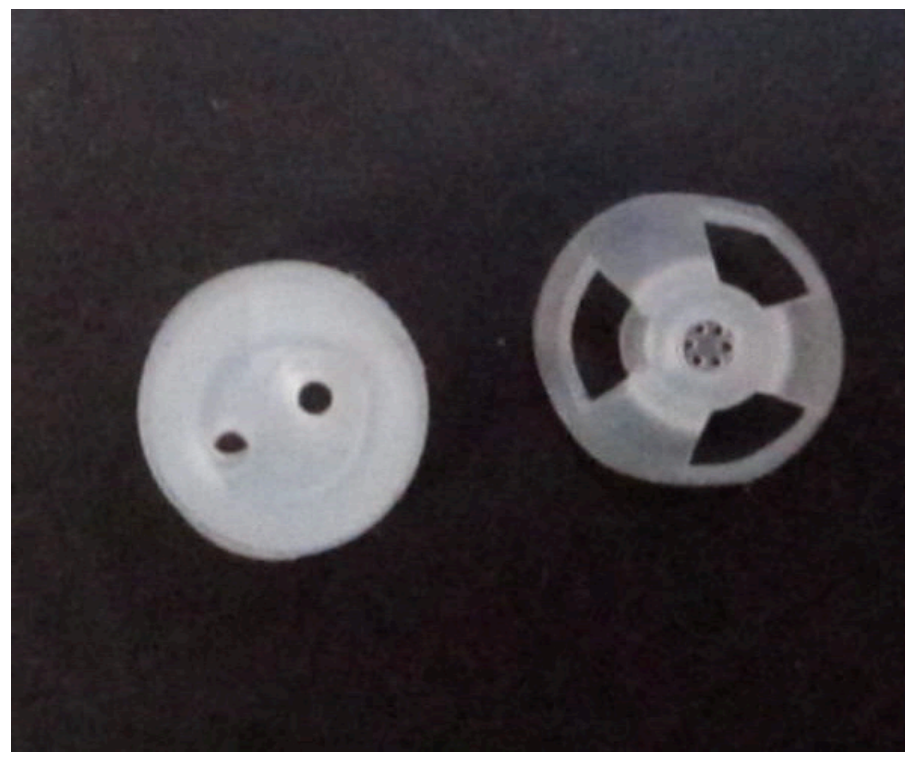

Figure 4-7. Picture micromold and open dome. The micromold is on the left, the open dome is on the right.

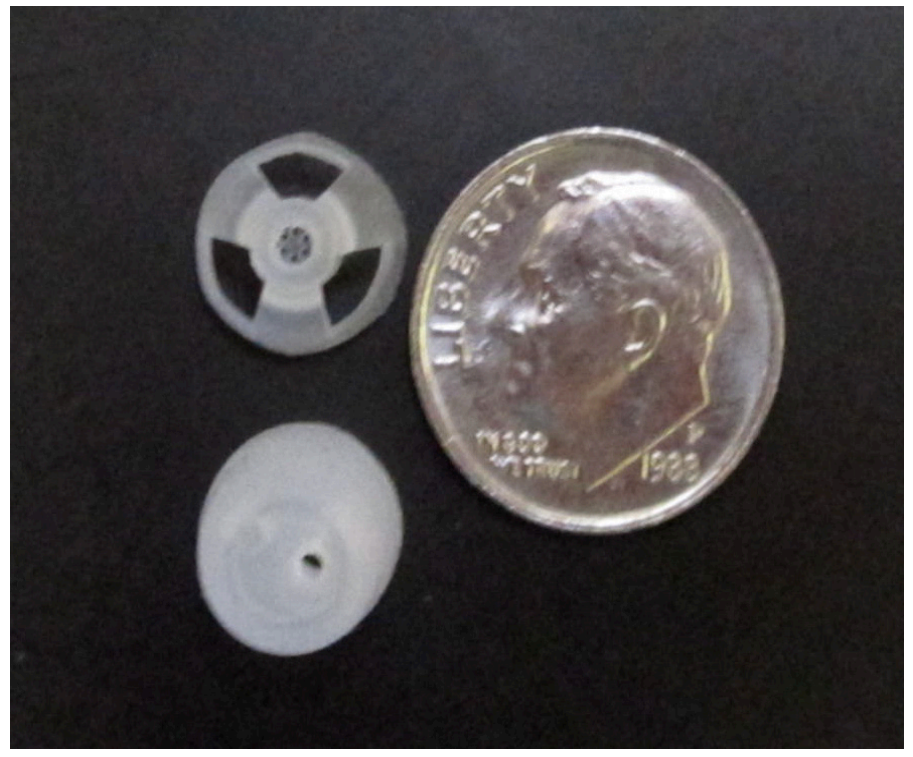

Figure 4-8. Picture of domes shown for size comparison to a dime. 


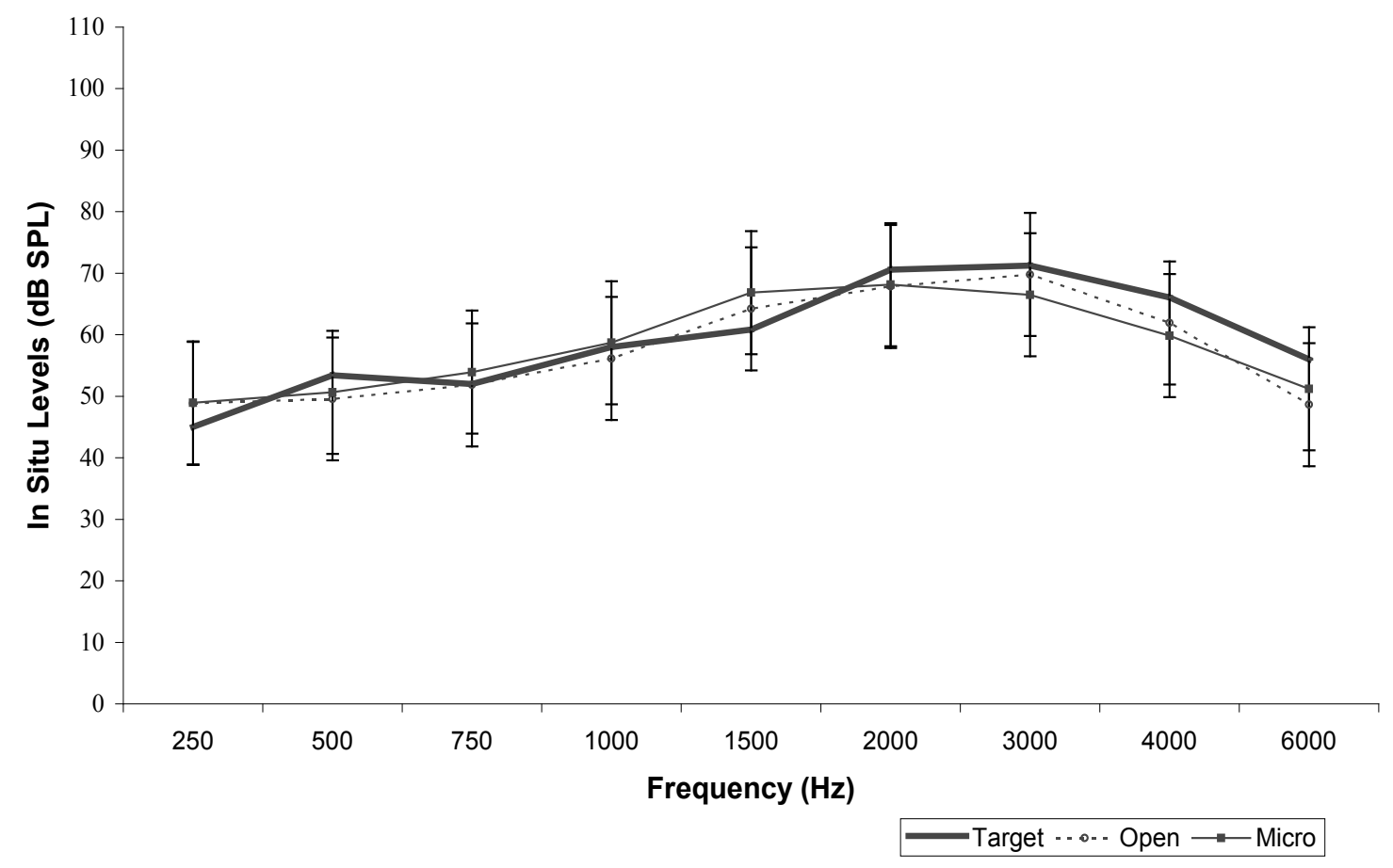

Figure 4-9. Mean probe microphone measures averaged across ears for each dome condition (input signal level $=55 \mathrm{~dB}$ SPL). 


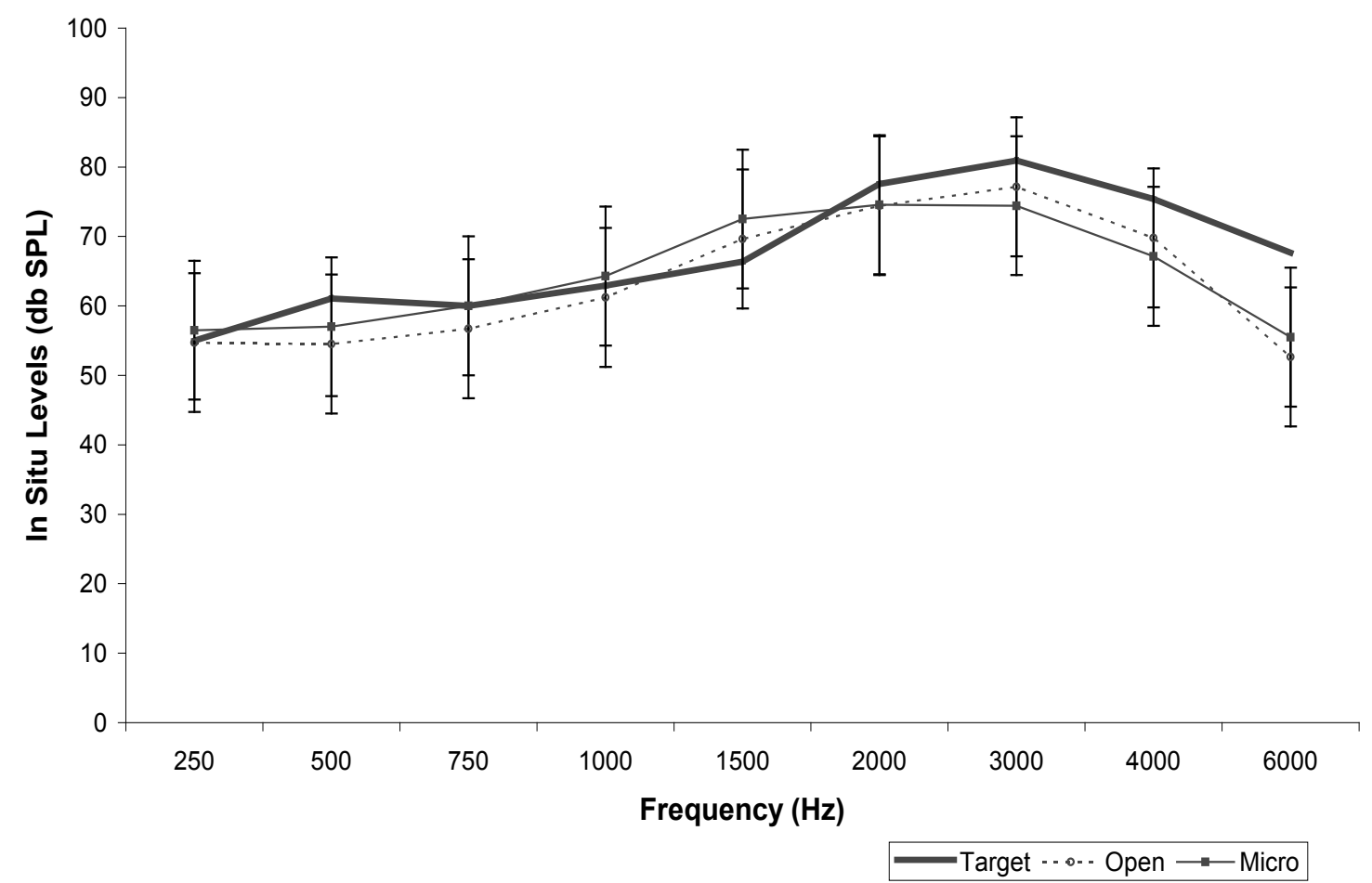

Figure 4-10. Mean probe microphone measures averaged across ears for each dome condition (input signal level $=65 \mathrm{~dB}$ SPL). 


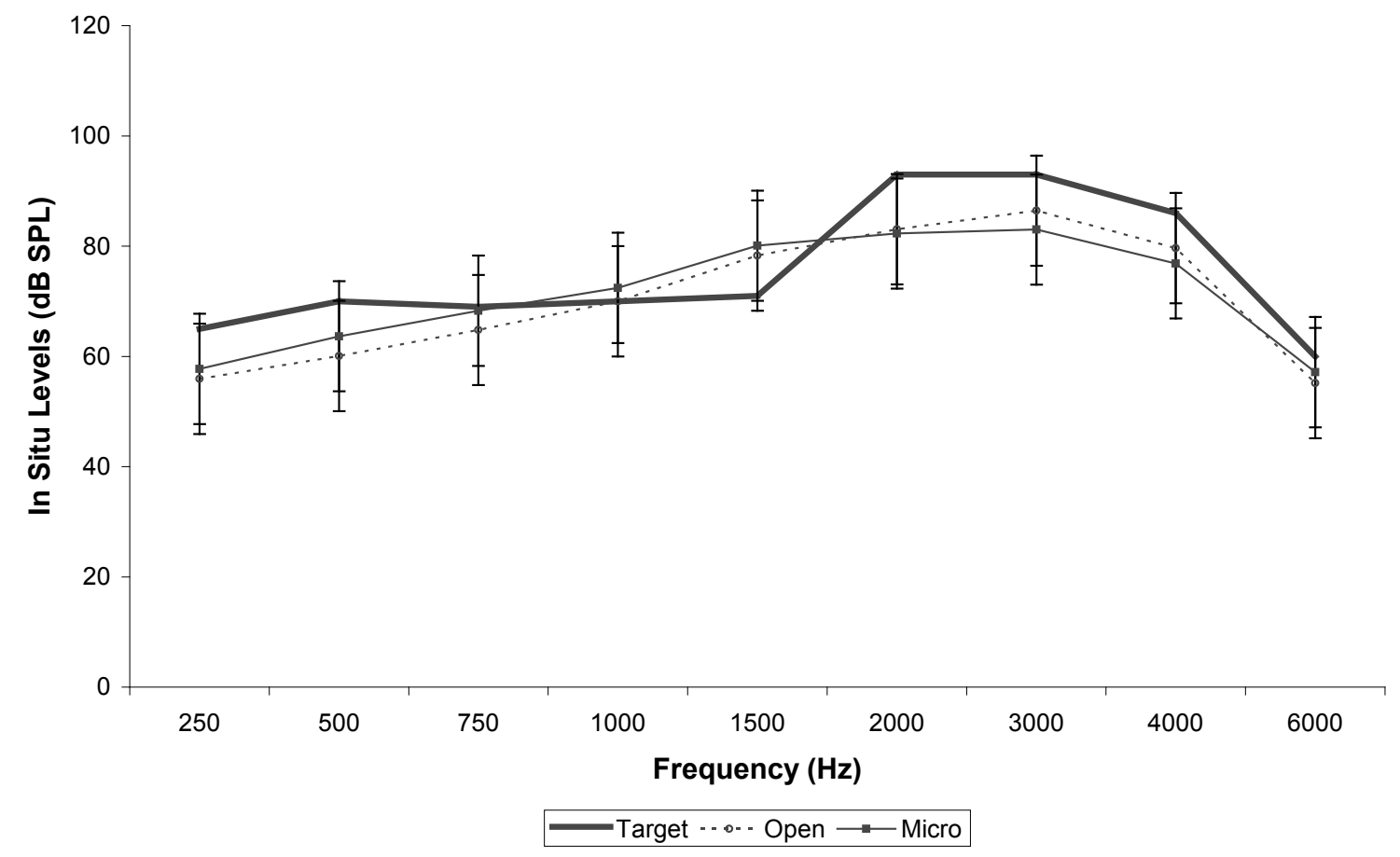

Figure 4-11. Mean probe microphone measures averaged across ears for each dome condition (input signal level $=75 \mathrm{~dB}$ SPL). 


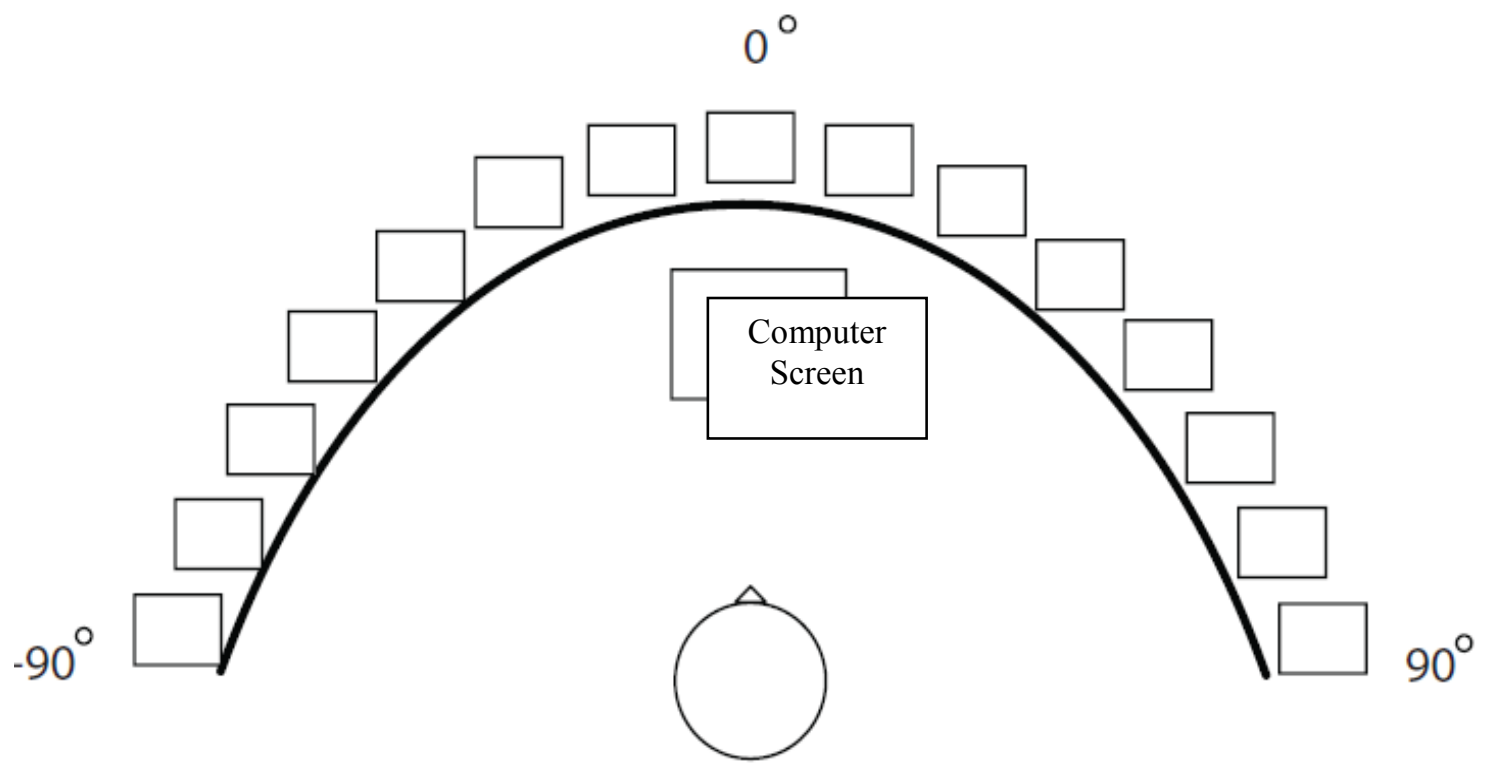

Figure 4-12. Diagram of test environment with speaker array. 
"baseball" originated by clicking on the picture on the computer screen that matched the picture below the loudspeaker from which the sound was believed to have originated. If the participant was unable to control the mouse (e.g. arthritis), the participant verbally reported which picture and the investigator clicked the picture in which the participant indicated. After each response, feedback was provided such that the correct-location picture flashed on the computer screen. The stimulus, "baseball," was randomly presented a total of ten times to each of the fifteen loudspeakers for a total of 150 trials per experimental condition. During individual trials, the participants were reminded to orient their head toward $0^{\circ}$ azimuth. If noticeable head movement occurred, the data for that trial was discarded and an additional trial was presented on that condition. 


\section{CHAPTER 5. RESULTS}

Prior to statistical analysis localization acuity for each participant was computed. The measure that was used to determine sound source localization acuity was done by comparing the actual loudspeaker location to the reported loudspeaker. Angle error scores were determined by computing the difference between the objective and subjective angular locations of the sound source. The value was computed for each loudspeaker location for each of the ten trials. An RMS error term was then computed for each loudspeaker location (angle of separation). The RMS error data were used to compute all statistics reported in the following results. For all RMS error information for each participant within all conditions, please refer to Appendix A, Table A-1.

\section{Age}

A paired-samples $t$-test was conducted to evaluate whether age affected horizontal localization ability controlling for family-wise error rate across the tests at the 0.05 level, using Holm's sequential Bonferroni procedure. Paired samples statistics are displayed in Table 5-1. The results indicated that the mean error for young normal hearing adult participants $(\mathrm{M}=3.37, \mathrm{SD}=.98)$ was significantly different than the mean for $>50$ normal hearing adult participants $(\mathrm{M}=6.85 ; \mathrm{SD}=2.57)$. The mean error for young normal hearing adult participants $(\mathrm{M}=3.47 ; \mathrm{SD}=.99)$ was significantly smaller than the mean for $>50$ hearing impaired adult participants $(\mathrm{M}=8.70$; $\mathrm{SD}=3.85)$. However, the mean error for $>50$ normal hearing adult participants $(\mathrm{M}=6.38 ; \mathrm{SD}=2.23)$ was not significantly different than the unaided (unamplified) mean for $>50$ hearing impaired adult participants $(\mathrm{M}=8.70 ; \mathrm{SD}=3.85)$ as shown in Table 5-2. These results indicate that young normal hearing adult participants performed significantly better than adults age 50 or older with or without impaired hearing on unaided (unamplified) localization tasks, and that adults age 50 or older with normal hearing did not perform significantly better than adults age 50 or older with hearing impairment unaided (see Appendix B: Figure B-7). Please refer to Appendix B: Figures B-1 through B-7 that display individual and group unaided (unamplified) average RMS error for all participants are plotted.

To further analyze the effect of age on localization acuity, correlation coefficients were computed among the three age groups in the unaided (unamplified) condition. The results of the correlational analyses presented in Table 5-3 show that correlations were statistically significant and were greater than or equal to .664 (see Figure B-6). Correlation coefficients were then computed between the $>50$ normal hearing participant group and the hearing impaired participant group. The results of this correlational analyses is presented in Table 5-4 and show that correlations were not statistically significant in unaided (unamplified) performance between the $>50$ normal hearing group and the hearing impaired group. 
Table 5-1. Paired-samples statistics.

\begin{tabular}{cccccc}
\hline \multirow{2}{*}{ Pair } & Group & Mean & $\mathrm{N}$ & $\begin{array}{c}\text { Standard. } \\
\text { Deviation }\end{array}$ & $\begin{array}{c}\text { Standard } \\
\text { Error Mean }\end{array}$ \\
\hline Pair 1 & Unaided Young & 3.38 & 10 & 0.98 & 0.31 \\
& Unaided >50 & 6.85 & 10 & 2.57 & 0.81 \\
Pair 2 & Unaided Young & 3.47 & 9 & 0.99 & 0.33 \\
& Unaided Hearing & & & & \\
& Impaired & 8.7 & 9 & 3.85 & 1.29 \\
Pair 3 & Unaided >50 & 6.38 & 9 & 2.23 & 0.74 \\
& Unaided Hearing & & & & \\
& Impaired & 8.7 & 9 & 3.86 & 1.29 \\
\hline
\end{tabular}

Note: $\mathrm{N}=$ sample size.

Table 5-2. Paired-samples t-test results for RMS error between participant groups.

\begin{tabular}{|c|c|c|c|c|c|}
\hline Pair & Group & $\begin{array}{c}\text { Mean } \\
\text { Difference }\end{array}$ & $\begin{array}{c}\text { Standard } \\
\text { Deviation }\end{array}$ & $\mathrm{t}$ & $\mathrm{p}$ \\
\hline \multirow[t]{2}{*}{ Pair 1} & Unaided Young & & & & \\
\hline & Unaided >50 & -3.47 & 2.63 & -4.18 & 0.002 \\
\hline \multirow[t]{2}{*}{ Pair 2} & Unaided Young & & & & \\
\hline & $\begin{array}{l}\text { Unaided Hearing } \\
\text { Impaired }\end{array}$ & -5.23 & 3.48 & -4.51 & 0.002 \\
\hline \multirow[t]{2}{*}{ Pair 3} & Unaided >50 & & & & \\
\hline & $\begin{array}{l}\text { Unaided Hearing } \\
\text { Impaired }\end{array}$ & -2.32 & 3.46 & -2.01 & 0.079 \\
\hline
\end{tabular}

Notes: $\mathrm{t}=$ variance $; \mathrm{p}=$ statistical significance. Italics denote significance. 
Table 5-3. Correlation of age and RMS error across all participant groups.

\begin{tabular}{cccc}
\hline Group & Correlation & Age & RMS Unaided \\
\hline Age & Pearson Correlation & 1 & 0.664 \\
& Significance (2-tailed) & & 0.000 \\
RMS Unaided & N & 30 & 30 \\
& Pearson Correlation & 0.664 & 1 \\
& Significance (2-tailed) & 0.000 & \\
& $\mathrm{~N}$ & 30 & 30 \\
\hline
\end{tabular}

Note: $\mathrm{RMS}=$ root mean squared; $\mathrm{N}=$ sample size. ${ }^{* *}$ Correlation is significant at the 0.01 level (2-tailed). Italics denote significance.

Table 5-4. Correlation of age and RMS error between $>50$ years and hearing impaired group.

\begin{tabular}{cccc}
\hline Group & Correlation & Age & RMS Unaided \\
\hline Age & Pearson Correlation & 1 & 0.361 \\
& Significance (2-tailed) & & 0.141 \\
& N & 18 & 18 \\
RMS Unaided & Pearson Correlation & 0.361 & 1 \\
& Significance (2-tailed) & 0.141 & \\
& N & 18 & 18 \\
\hline
\end{tabular}

Note: $\mathrm{N}=$ sample size. 


\section{Microphone and Dome Technology}

A two way repeated measures analysis of variance (ANOVA) was conducted to evaluate the effects of two dome types and three microphone configurations on localization acuity. The dependent variable was the RMS error. The within-subjects factors were dome with two levels (open and micromold) and microphone configuration with three levels (omnidirectional, adaptive directional, and fixed directional). The means and standard deviations for RMS error as a function of the two factors are presented in Table 5-5. The ANOVA indicated no significant main effects for microphone configuration, $\left[\underline{F}(2,16)=.382, \underline{p}=.689\right.$, partial $\left.\mathrm{y}^{2}=.046, \Omega=.101\right]$ or microphone by dome interaction $\left[\mathrm{F}(2,16)=.028, \mathrm{p}=.973\right.$, partial $\left.\mathrm{n}^{2}=.003, \Omega=.053\right]$; however, the dome main effect was significant $[\mathrm{F}(1,8)=8.361, \underline{\mathrm{p}}=.020$, , partial $\left.\mathrm{y}^{2}=.511, \Omega=.717\right]$ as evidenced in Table 5-6. Overall, results indicated that no significant effects were evident for microphone configuration or microphone configuration by dome interaction (see Figure B-8); however a significant main effect was revealed for dome type. Please refer to Appendix B, Figures B-8 through B-19 that plot individual average RMS error for all hearing impaired participants within each microphone and dome condition. See Appendix B, Figures B-20 through B-28 displaying plots for each condition within a participant.

A one-way within-subjects ANOVA was conducted with the factor of dome with three levels (no dome, open dome, micromold) and the dependent variable being RMS error to evaluate the effect of mold to baseline (unamplified) localization acuity. The results for the ANOVA indicated no significant effect $[\underline{F}(1,8)=3.709, p=.08$, multivariate $\mathrm{y}^{2}=5.15$ ] and pairwise comparisons (see Table 5-7) controlling for familywise error rate across the tests at the 0.05 level, using the Holm's sequential Bonferroni procedure. These results suggest that although there was a significant dome effect within the two way repeated measures ANOVA, that this effect may be small and should be interpreted with caution. 
Table 5-5. Means and standard deviations for RMS error.

\begin{tabular}{ccccc}
\hline \multirow{2}{*}{ Dome } & Microphone & Mean & $\begin{array}{c}\text { Standard } \\
\text { Deviation }\end{array}$ & $\mathrm{N}$ \\
\hline \multirow{4}{*}{ Open } & Adaptive & & & \\
& Directional & 9.14 & 4.04 & 9 \\
& Fixed Directional & 9.36 & 3.97 & 9 \\
& Omnidirectional & 8.95 & 4.19 & 9 \\
& Adaptive & & & \\
& Directional & 9.71 & 4.48 & 9 \\
& Fixed Directional & 9.75 & 3.57 & 9 \\
\hline
\end{tabular}

Note: $\mathrm{N}=$ sample size.

Table 5-6. ANOVA results for localization acuity.

\begin{tabular}{cccccc}
\hline Technology & $\mathrm{F}$ & $\mathrm{df}$ & $\mathrm{p}$ & partial $\mathrm{y}^{2}$ & $\Omega$ \\
\hline Dome & 8.361 & 1,8 & 0.02 & 0.511 & 0.717 \\
Microphone & 0.382 & 2,16 & 0.689 & 0.046 & 0.101 \\
$\begin{array}{c}\text { Dome x } \\
\text { Microphone }\end{array}$ & 0.028 & 2,16 & 0.973 & 0.003 & 0.053 \\
\hline
\end{tabular}

Notes: $\mathrm{F}=$ frequency; $\mathrm{df}=$ degree of freedom; $\mathrm{p}=$ statistical significance; partial $\mathrm{n}^{2}=$ proportion of total variation attibutal to the factor; $\Omega=$ amount of variation. Italics denote significance. 
Table 5-7. One-way within-subject ANOVA results for dome.

\begin{tabular}{ccccccc}
\hline & & & & & \multicolumn{2}{c}{$\begin{array}{c}95 \% \text { Confidence Interval } \\
\text { for Difference(a) }\end{array}$} \\
\cline { 5 - 7 } Condition & Occlusion & Difference & $\begin{array}{c}\text { Standard } \\
\text { Error }\end{array}$ & Sig.(a) & $\begin{array}{c}\text { Lower } \\
\text { Bound }\end{array}$ & $\begin{array}{c}\text { Upper } \\
\text { Bound }\end{array}$ \\
\hline \multirow{2}{*}{ Unaided } & Open & -0.449 & 0.433 & 0.989 & -1.754 & 0.856 \\
& Micromold & -0.918 & 0.523 & 0.352 & -2.496 & 0.660 \\
\multirow{5}{*}{ Open } & Unaided & 0.449 & 0.433 & 0.989 & -0.856 & 1.754 \\
& Micromold & -0.469 & 0.162 & 0.059 & -0.956 & 0.018 \\
& Unaided & 0.918 & 0.523 & 0.352 & -0.660 & 2.496 \\
& Open & 0.469 & 0.162 & 0.059 & -0.018 & 0.956 \\
\hline
\end{tabular}

Notes: Data based on estimated marginal means. Sig(a) = Adjusted significance for multiple comparisons using the Holm's Bonferroni procedure. 


\section{CHAPTER 6. DISCUSSION}

One purpose of the present study was to determine if age affects azimuthal localization acuity in adult listeners. Previous research has failed to control for age and has compared the performance of older hearing impaired listeners to young normal hearing adult listeners (Noble et al, 1994; Van den Bogaert et al., 2006; Chung et al., 2008). Prior work examining neurophysiological responses have suggested that overall responsiveness of the central auditory system and age are correlated (Polich, 2004; Harkrider et al., 2005); consequently, it was reasonable to hypothesize that age may affect localization acuity as part of the aging process in adults. Results of this study suggest that the normal aging process may affect azimuthal localization ability as young normal hearing adults listeners performed significantly better than older normal hearing adult listeners. These results are consistent with prior research by Viehweg and colleagues (1960) that examined localization ability in listeners with unilateral hearing loss. Within this study, participants were divided into 6 age groups based upon current decade of life (first through sixth). Results of this study indicated a significant difference in performance between age groups; however, due to methodological problems and data collection data from this study must be interpreted with extreme caution.

Further analysis of age within the present study revealed that older normal hearing adult listeners did not perform significantly better than their unaided hearing impaired peers. Hearing impaired participants within this study had normal or near normal low frequency hearing. Wightman and Kistler (1992) suggested that when interaural time cues (low frequency information) are present, that they 'override' interaural level cues. Thus, it is possible to speculate that no statistical differences were evident between the older normal hearing group and the hearing impaired group due to normal low frequency hearing in both groups. Interestingly, the oldest participant in this study had the best localization ability when compared between both older participant groups (normal hearing and hearing impaired). Further examination revealed that this participant also had the best low frequency pure-tone average out of both older participant groups. Taken together, these results warrant further exploration; however, it is possible to speculate that a relationship exists between age and low frequency hearing on localization tasks.

Another purpose of this study was to examine the effect of microphone technology on azimuthal localization when amount of occlusion (type of dome) is controlled. Prior work has examined microphone technologies without controlling for manufacturer or occlusion (Noble et al., 1998; Van den Bogaert et al., 2006). Results indicated no significant differences between microphone configurations (omnidirectional, adaptive directional, fixed directional). It should be noted that all testing was completed in quiet; therefore, we cannot postulate that the microphone 1 hearing instruments, on localization ability. It has been shown that traditional earmolds without venting, degrade localization performance within a listener and that although vented performance is better than unvented, it is not beneficial when localizing in the horizontal plane (Byrne et al., 1998; Noble et al., 1998). Open-fit hearing aids provide flexible fitting options and are considered 'unoccluding' compared to traditional, custom fit, earmolds. These hearing 
aids have several 'dome' options to choose from, all of which are considered 'unoccluding' as they are not custom products. Initial results of this study indicated a significant difference in participant performance between dome types; however, further examination revealed that no significant differences between dome types and no significant differences between dome type and unaided. These results should be interpreted with caution. Although a significant effect was initially noted between domes, it was later discounted. Simply stated, although a difference in performance was evident, it was slight. This suggests that both domes types maintain localization acuity within a listener relative to their performance unaided.

The final purpose of this study was to determine if a synergistic effect existed between microphone configuration and dome type within open-fit hearing aids on horizontal localization ability within a listener. No significant results were noted, thereby suggesting that no degrading effects were evident comparative to baseline (unaided) measures. Furthermore, as localization acuity was similar between older groups (normal hearing and hearing impaired) it can be assumed that localization performance with openfit hearing aids was also not significantly different from their normal hearing peers for the conditions tested within this study. 


\section{CHAPTER 7. CONCLUSIONS}

Overall, results of this study did not indicate a degrading effect on a participant's localization acuity when fit with open-fit hearing instruments in the conditions tested. Neither microphone configuration, dome type, nor a combination of the two, severely degraded localization acuity within a participant. Even so, these results should be interpreted with caution as all participants in this study were evaluated in quiet with all other features within the hearing instruments deactivated.

Other findings of this study indicated that age significantly impacted localization acuity. However, no differences in localization acuity were noted between $>50$ normal hearing participants and hearing impaired participants. Thus, it may be postulated, that this finding may be due to exceptional low frequency hearing sensitivity in each group. All findings should be taken with caution, in that all participants in this study were evaluated in quiet with all other features of the hearing aid deactivated. Furthermore, all results of this study are limited to hearing instruments within the same manufacturer and are limited to small sample size. Consequently, future research should examine these effects in noise, with other features activated, across manufactures in a larger sample size.

This study was approved by the Institutional Review Board at the University of Tennessee Health Sciences Campus. All participants were counseled regarding the nature of the study and signed an informed consent form (see Appendix C) prior to their participation. 


\section{LIST OF REFERENCES}

Alworth, L.N., Plyler, P.N., Reber, M.B., \& Johnstone, P.M. (2010). The effects of receiver placement on probe microphone, performance, and subjective measures with open canal hearing instruments. Journal of the American Academy of Audiology. 21(4): 249-266.

American National Standards Institute. (1991). Criteria for Permissible Ambient Noise during Audiometric Testing (ANSI S3.1-1991). New York: American National Standards Institute.

American National Standards Institute. (1996). American National Standards Specification for Audiometers (ANSI S3. 6-1996). New York. American National Standards Institute.

Best, V., Carlile, S., Jin, C., \& van Schaik, A. (2005). The role of high frequencies in speech localization. Journal of the Acoustical Society of America. 118: 353-363.

Bronkhorst, A.W. (1995). Localization of real and virtual sound sources. Journal of the Acoustical Society of America. 95(3): 1534-1547.

Bronkhorst, A.W. \& Plomp, R. (1989). Binaural speech intelligibility in noise for hearing impaired listeners. Journal of the Acoustical Society of America. 86(4): 1374-1383.

Byrne, D., Dillon, H., Ching, T., Katsch, R., \& Keidser, G. (2001). NAL-NL1 procedure for fitting nonlinear hearing aids: characteristics and comparisons with other procedures. Journal of the American Academy of Audiology. 12: 37-51.

Byrne, D., Sinclair, S., \& Noble, W. (1998). Open earmold fittings for improving aided auditory localization for sensorineural hearing losses with good high-frequency hearing. Ear and Hearing. 19(1): 62-71.

Chung, K., Neuman, A.C., \& Higgins, M. (2008). Effects of in-the-ear microphone directionality on sound direction identification. Journal of the Acoustical Society of America. 123(4): 2264-2275.

Dillon, H. (2001). Hearing Aids. New York: Thieme, 24-28.

Drennan, W.R., Gatehouse, S., Howell, P., van Tasell, D., \& Lund, S. (2005). Localization and speech-identification ability of hearing-impaired listeners using phase-preserving amplification. Ear and Hearing. 26(5): 461-472.

Gelfand, S.A. (2004). HEARING An Introduction to Psychological and Physiological Acoustics. New York: Marcel Dekker, 395-398. 
Hausler, R., Colburn, S., \& Marr, E. (1983). Sound localization in subjects with impaired hearing. Acta Otolaryngology. 400: 1-62.

Keidser, G., Rohrseitz, K., Dillon, H., Hamacher, V., Carter, L., Rass, U., \& Convery, E. (2006). The effect of multi-channel wide dynamic range compression, noise reduction, and the directional microphone on horizontal localization performance in hearing aid wearers. International Journal of Audiology. 45: 563-579.

Kochkin, S. (2005). MarkeTrak VII: Hearing loss population tops 31 million people. The Hearing Journal. 50: 28-32.

Langendijk, E.H.A., \& Bronkhorst, A.W. (2002). Contribution of spectral cues to human sound localization. Journal of the Acoustical Society of America. 112(4): 1583-1596.

Markous, J.C., \& Middlebrooks, J.C. (1990). Two-dimensional sound localization by human listeners. Journal of the Acoustical Society of America. 87(5): 2188-2200.

Musicant, A. \& Butler, R. (1984). The influence of pinnae-based spectral cues on sound localization. Journal of the Acoustical Society of America. 75: 1195-1200.

Noble, W., \& Byrne, D. (1990). A comparison of different binaural hearing aid systems for sound localization in the horizontal and vertical planes. British Journal of Audiology. 24: 335-346.

Noble, W., Byrne, D., \& Lepage, B. (1994). Effects on sound localization of configuration and type of hearing impairment. Journal of the Acoustical Society of America. 95(2): 992-1005.

Noble, W., Sinclair, S., \& Byrne, D. (1998). Improvements in aided sound localization with open earmolds: observations in people with high-frequency hearing loss. Journal of the Acoustical Society of America. 9(1): 25-34.

Polich, J. (2004). Clinical application of the P300 event-related brain potential. Physical Medicine and Rehabilitation Clinics of North America. 15: 133-161.

Souza, P.E., \& Turner, C.W. (1994). Masking of speech in young and elderly listeners with hearing loss. Journal of Speech Language and Hearing Research. 37: 655661.

Van den Bogaert, T., Klasen, T.J., Moonen, M., van Deun, L., \& Wouters, J. (2006). Horizontal localization with bilateral hearing aids: without is better than with. Journal of the Acoustical Society of America. 119(1): 515-526.

Viehweg, R., \& Campbell, R. (1960). Localization difficulty in monaurally impaired listeners. The Annals of Otology, Rhinology, \& Laryngology. 69: 622-634. 
Wightman, F.L., \& Kistler, D.J. (1992). The dominant role of low-frequency interaural time differences in sound localization. Journal of the Acoustical Society of America. 91(3): 1648-1661. 
APPENDIX A. STATISTICAL TABLE 
Table A-1. RMS error values for each participant across each condition.

\begin{tabular}{|c|c|c|c|c|c|c|c|}
\hline Participant & Unaided & $\begin{array}{l}\text { Open } \\
\text { Omni }\end{array}$ & $\begin{array}{l}\text { Open } \\
\text { Adapt }\end{array}$ & $\begin{array}{l}\text { Open } \\
\text { Fixed }\end{array}$ & $\begin{array}{l}\text { Micro } \\
\text { Omni }\end{array}$ & $\begin{array}{l}\text { Micro } \\
\text { Adapt }\end{array}$ & $\begin{array}{l}\text { Micro } \\
\text { Fixed }\end{array}$ \\
\hline Young A & 3.07 & $\mathrm{n} / \mathrm{a}$ & $\mathrm{n} / \mathrm{a}$ & $\mathrm{n} / \mathrm{a}$ & $\mathrm{n} / \mathrm{a}$ & $\mathrm{n} / \mathrm{a}$ & $\mathrm{n} / \mathrm{a}$ \\
\hline Young B & 3.07 & $\mathrm{n} / \mathrm{a}$ & $\mathrm{n} / \mathrm{a}$ & $\mathrm{n} / \mathrm{a}$ & $\mathrm{n} / \mathrm{a}$ & $\mathrm{n} / \mathrm{a}$ & $\mathrm{n} / \mathrm{a}$ \\
\hline Young C & 4.48 & $\mathrm{n} / \mathrm{a}$ & $\mathrm{n} / \mathrm{a}$ & $\mathrm{n} / \mathrm{a}$ & $\mathrm{n} / \mathrm{a}$ & $\mathrm{n} / \mathrm{a}$ & $\mathrm{n} / \mathrm{a}$ \\
\hline Young D & 2.43 & $\mathrm{n} / \mathrm{a}$ & $\mathrm{n} / \mathrm{a}$ & $\mathrm{n} / \mathrm{a}$ & $\mathrm{n} / \mathrm{a}$ & $\mathrm{n} / \mathrm{a}$ & $\mathrm{n} / \mathrm{a}$ \\
\hline Young E & 2.84 & $\mathrm{n} / \mathrm{a}$ & $\mathrm{n} / \mathrm{a}$ & $\mathrm{n} / \mathrm{a}$ & $\mathrm{n} / \mathrm{a}$ & $\mathrm{n} / \mathrm{a}$ & $\mathrm{n} / \mathrm{a}$ \\
\hline Young F & 2.48 & $\mathrm{n} / \mathrm{a}$ & $\mathrm{n} / \mathrm{a}$ & $\mathrm{n} / \mathrm{a}$ & $\mathrm{n} / \mathrm{a}$ & $\mathrm{n} / \mathrm{a}$ & $\mathrm{n} / \mathrm{a}$ \\
\hline Young G & 3.79 & $\mathrm{n} / \mathrm{a}$ & $\mathrm{n} / \mathrm{a}$ & $\mathrm{n} / \mathrm{a}$ & $\mathrm{n} / \mathrm{a}$ & $\mathrm{n} / \mathrm{a}$ & $\mathrm{n} / \mathrm{a}$ \\
\hline Young $\mathrm{H}$ & 3.59 & $\mathrm{n} / \mathrm{a}$ & $\mathrm{n} / \mathrm{a}$ & $\mathrm{n} / \mathrm{a}$ & $\mathrm{n} / \mathrm{a}$ & $\mathrm{n} / \mathrm{a}$ & $\mathrm{n} / \mathrm{a}$ \\
\hline Young I & 5.48 & $\mathrm{n} / \mathrm{a}$ & $\mathrm{n} / \mathrm{a}$ & $\mathrm{n} / \mathrm{a}$ & $\mathrm{n} / \mathrm{a}$ & $\mathrm{n} / \mathrm{a}$ & $\mathrm{n} / \mathrm{a}$ \\
\hline Young J & 2.59 & $\mathrm{n} / \mathrm{a}$ & $\mathrm{n} / \mathrm{a}$ & $\mathrm{n} / \mathrm{a}$ & $\mathrm{n} / \mathrm{a}$ & $\mathrm{n} / \mathrm{a}$ & $\mathrm{n} / \mathrm{a}$ \\
\hline Young K & 4.9 & $\mathrm{n} / \mathrm{a}$ & $\mathrm{n} / \mathrm{a}$ & $\mathrm{n} / \mathrm{a}$ & $\mathrm{n} / \mathrm{a}$ & $\mathrm{n} / \mathrm{a}$ & $\mathrm{n} / \mathrm{a}$ \\
\hline$>50 \mathrm{~A}$ & 4.39 & $\mathrm{n} / \mathrm{a}$ & $\mathrm{n} / \mathrm{a}$ & $\mathrm{n} / \mathrm{a}$ & $\mathrm{n} / \mathrm{a}$ & $\mathrm{n} / \mathrm{a}$ & $\mathrm{n} / \mathrm{a}$ \\
\hline$>50 \mathrm{~B}$ & 6.77 & $\mathrm{n} / \mathrm{a}$ & $\mathrm{n} / \mathrm{a}$ & $\mathrm{n} / \mathrm{a}$ & $\mathrm{n} / \mathrm{a}$ & $\mathrm{n} / \mathrm{a}$ & $\mathrm{n} / \mathrm{a}$ \\
\hline$>50 \mathrm{C}$ & 6.11 & $\mathrm{n} / \mathrm{a}$ & $\mathrm{n} / \mathrm{a}$ & $\mathrm{n} / \mathrm{a}$ & $\mathrm{n} / \mathrm{a}$ & $\mathrm{n} / \mathrm{a}$ & $\mathrm{n} / \mathrm{a}$ \\
\hline$>50 \mathrm{D}$ & 5.38 & $\mathrm{n} / \mathrm{a}$ & $\mathrm{n} / \mathrm{a}$ & $\mathrm{n} / \mathrm{a}$ & $\mathrm{n} / \mathrm{a}$ & $\mathrm{n} / \mathrm{a}$ & $\mathrm{n} / \mathrm{a}$ \\
\hline$>50 \mathrm{E}$ & 7.77 & $\mathrm{n} / \mathrm{a}$ & $\mathrm{n} / \mathrm{a}$ & $\mathrm{n} / \mathrm{a}$ & $\mathrm{n} / \mathrm{a}$ & $\mathrm{n} / \mathrm{a}$ & $\mathrm{n} / \mathrm{a}$ \\
\hline$>50 \mathrm{~F}$ & 8.01 & $\mathrm{n} / \mathrm{a}$ & $\mathrm{n} / \mathrm{a}$ & $\mathrm{n} / \mathrm{a}$ & $\mathrm{n} / \mathrm{a}$ & $\mathrm{n} / \mathrm{a}$ & $\mathrm{n} / \mathrm{a}$ \\
\hline$>50 \mathrm{G}$ & 4.65 & $\mathrm{n} / \mathrm{a}$ & $\mathrm{n} / \mathrm{a}$ & $\mathrm{n} / \mathrm{a}$ & $\mathrm{n} / \mathrm{a}$ & $\mathrm{n} / \mathrm{a}$ & $\mathrm{n} / \mathrm{a}$ \\
\hline$>50 \mathrm{H}$ & 3.59 & $\mathrm{n} / \mathrm{a}$ & $\mathrm{n} / \mathrm{a}$ & $\mathrm{n} / \mathrm{a}$ & $\mathrm{n} / \mathrm{a}$ & $\mathrm{n} / \mathrm{a}$ & $\mathrm{n} / \mathrm{a}$ \\
\hline$>50 \mathrm{I}$ & 10.76 & $\mathrm{n} / \mathrm{a}$ & $\mathrm{n} / \mathrm{a}$ & $\mathrm{n} / \mathrm{a}$ & $\mathrm{n} / \mathrm{a}$ & $\mathrm{n} / \mathrm{a}$ & $\mathrm{n} / \mathrm{a}$ \\
\hline$>50 \mathrm{~K}$ & 11.8 & $\mathrm{n} / \mathrm{a}$ & $\mathrm{n} / \mathrm{a}$ & $\mathrm{n} / \mathrm{a}$ & $\mathrm{n} / \mathrm{a}$ & $\mathrm{n} / \mathrm{a}$ & $\mathrm{n} / \mathrm{a}$ \\
\hline HI A & 6.66 & 8.4 & 8.04 & 8.97 & 13.14 & 7.29 & 8.12 \\
\hline HI B & 11.82 & 14.26 & 14.94 & 14.93 & 14.76 & 14.81 & 15.89 \\
\hline $\mathrm{HI} \mathrm{C}$ & 5.19 & 4.06 & 4.11 & 4.62 & 2.7 & 4.13 & 6.52 \\
\hline HI D & 3.79 & 4.64 & 4.27 & 4.97 & 4.81 & 5.64 & 6.46 \\
\hline HI E & 9.07 & 7.57 & 9.49 & 8.22 & 9.22 & 9.56 & 9.47 \\
\hline HI F & 7.2 & 7.27 & 8.27 & 7.91 & 6.8 & 9.52 & 8.1 \\
\hline HI G & 14.1 & 13.68 & 12.94 & 12.33 & 13.77 & 12.83 & 11.01 \\
\hline HI H & 6.2 & 5.93 & 6.23 & 7 & 6.67 & 6.26 & 7.08 \\
\hline HI I & 14.31 & 14.71 & 13.95 & 15.31 & 12.59 & 17.39 & 15.14 \\
\hline
\end{tabular}


APPENDIX B. FIGURES AND GRAPHS 


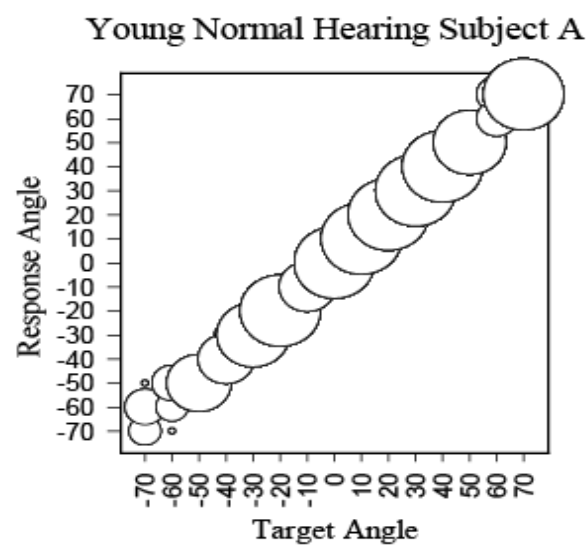

Young Normal Hearing Subject B

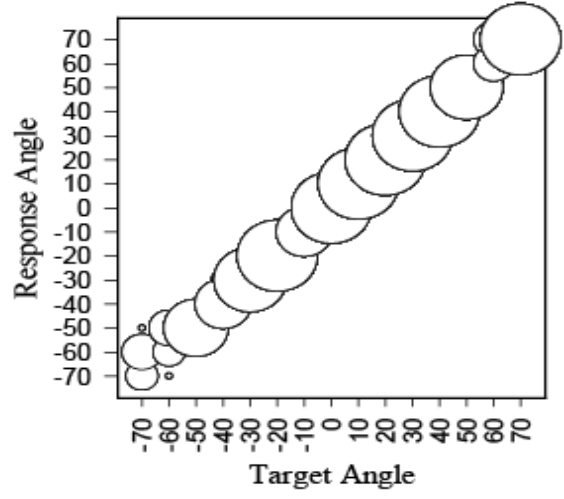

Young Normal Hearing Subject C

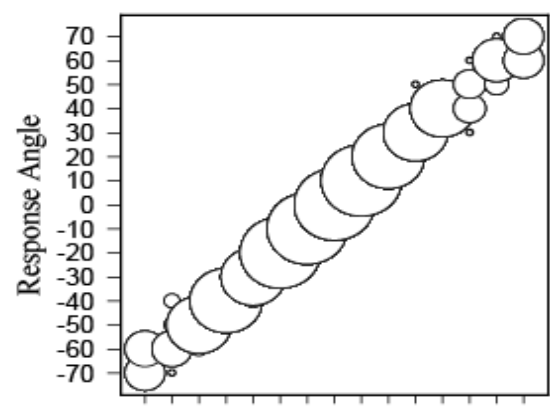

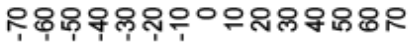

Target Angle
Young Normal Hearing Subject D

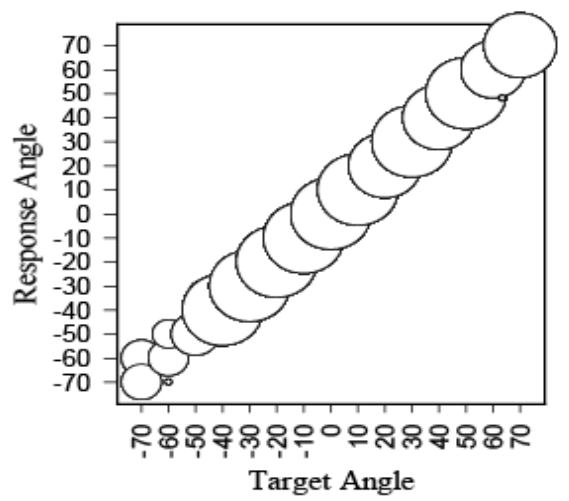

Young Normal Hearing Subject E

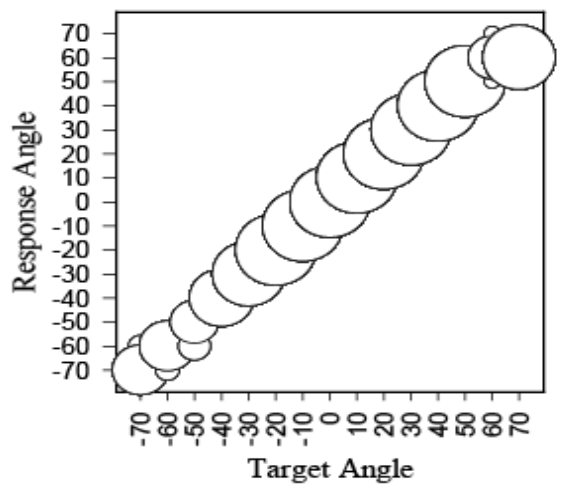

Young Normal Hearing Subject F

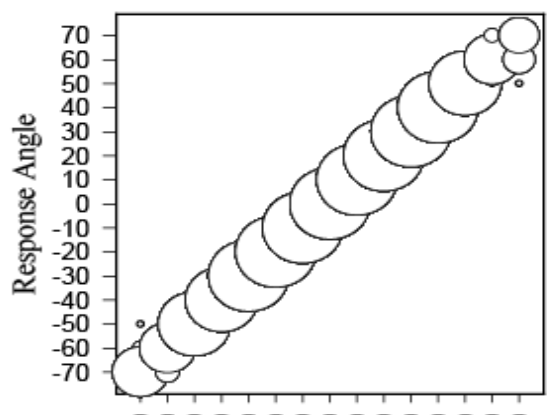

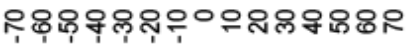

Target Angle

Figure B-1. Young Normal Hearing Participants (A-F) perceived angle plotted as a function of actual target angle of the stimuli. The bubble size indicates the number of times (out of ten trials) that a particular angle was chosen. Larger bubbles indicate an angle that was often perceived, whereas smaller bubbles indicate an angle was rarely perceived. 

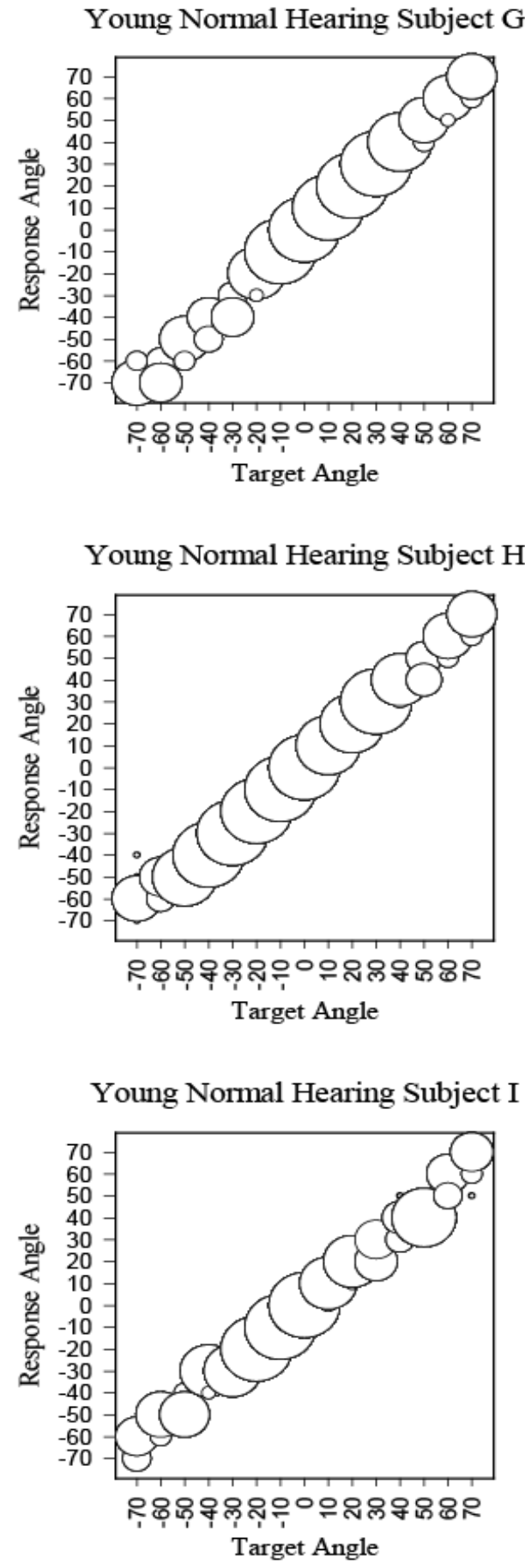
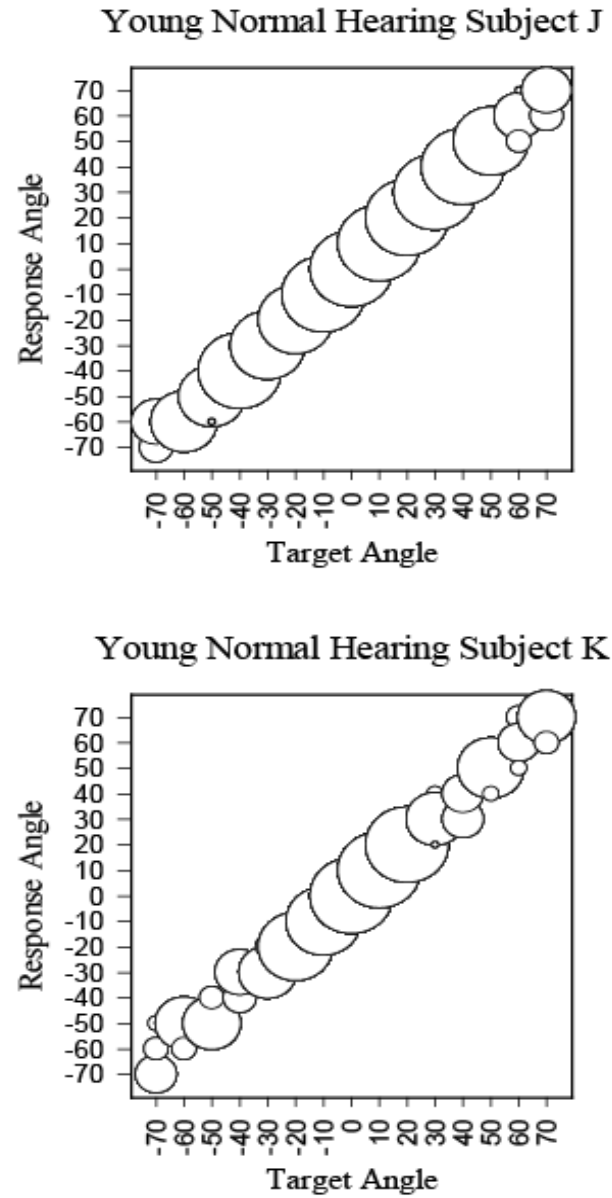

Figure B-2. Young Normal Hearing Participants (G-K) perceived angle plotted as a function of actual target angle of the stimuli. The bubble size indicates the number of times (out of ten trials) that a particular angle was chosen. Larger bubbles indicate an angle that was often perceived, whereas smaller bubbles indicate an angle was rarely perceived. 

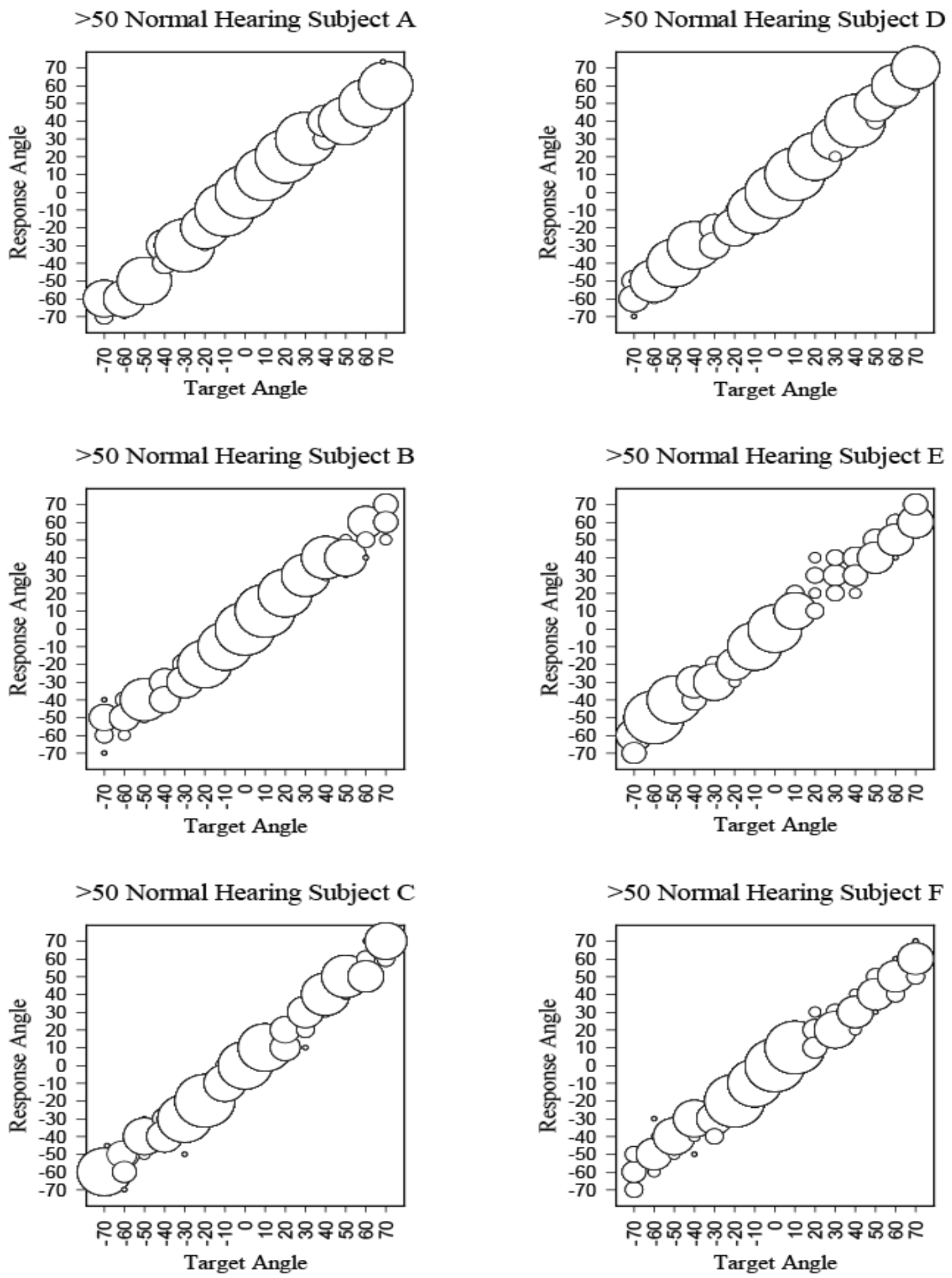

Figure B-3. $>50$ Normal Hearing Participants (A-F) perceived angle plotted as a function of actual target angle of the stimuli. The bubble size indicates the number of times (out of ten trials) that a particular angle was chosen. Larger bubbles indicate an angle that was often perceived, whereas smaller bubbles indicate an angle was rarely perceived. 

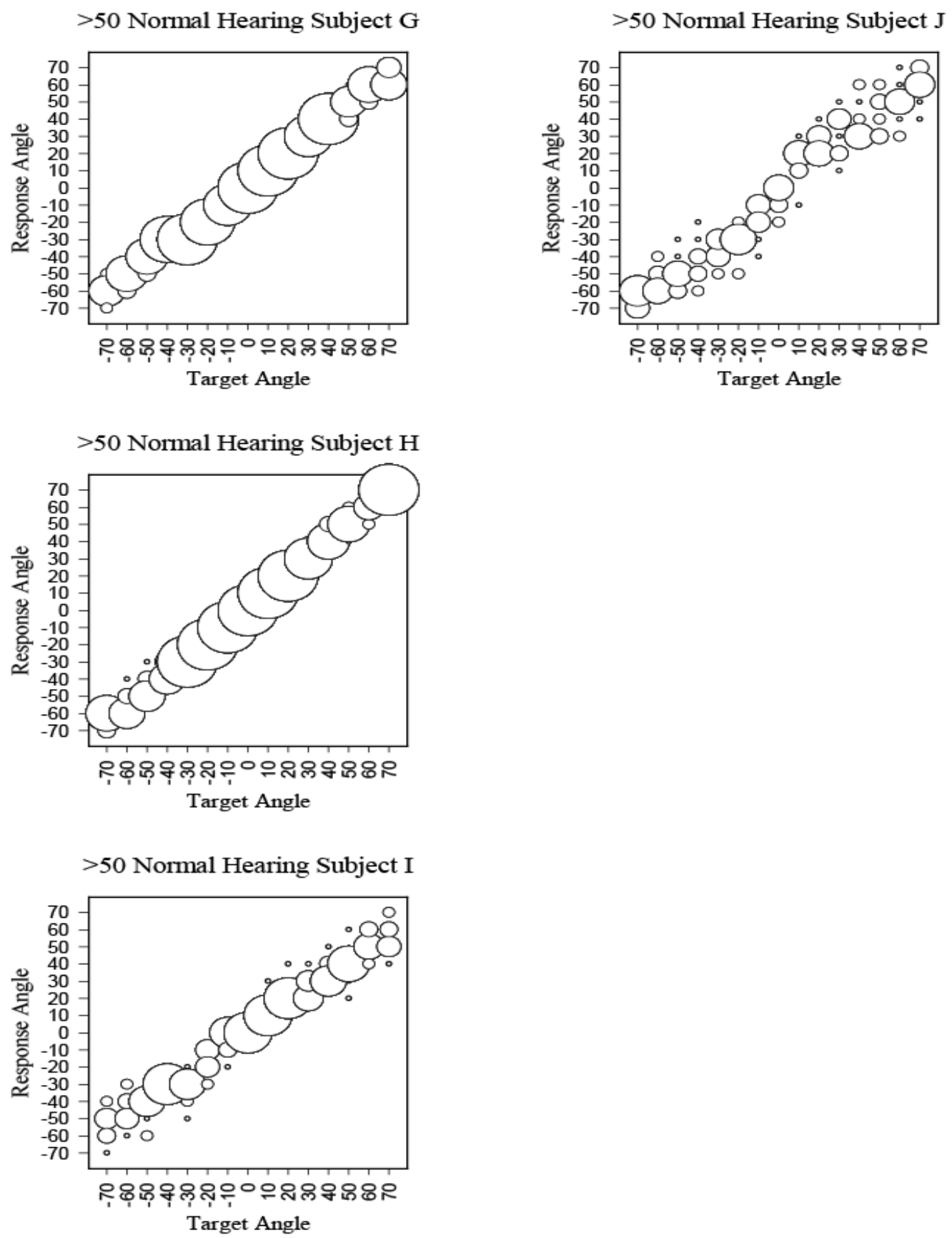

Figure B-4. $>\mathbf{5 0}$ Normal Hearing Participants (G-J) perceived angle plotted as a function of actual target angle of the stimuli. The bubble size indicates the number of times (out of ten trials) that a particular angle was chosen. Larger bubbles indicate an angle that was often perceived, whereas smaller bubbles indicate an angle was rarely perceived. 
HI Unaided Subject A

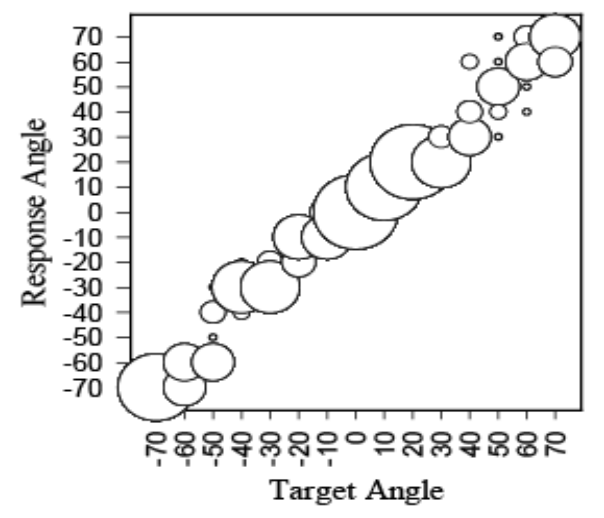

HI Unaided Subject B

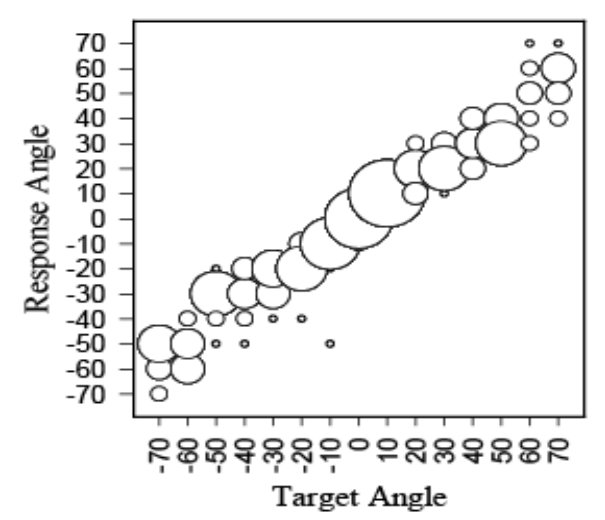

HI Unaided Subject C

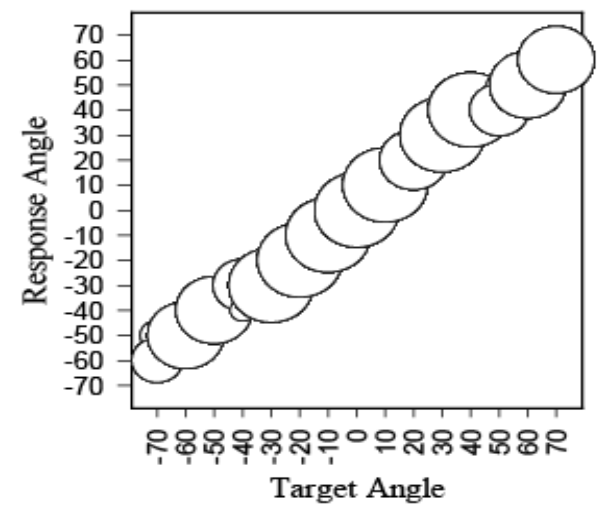

HI Unaided Subject D

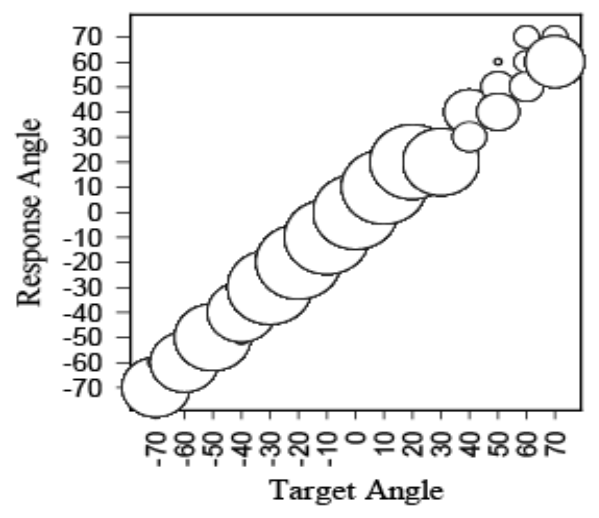

HI Unaided Subject E

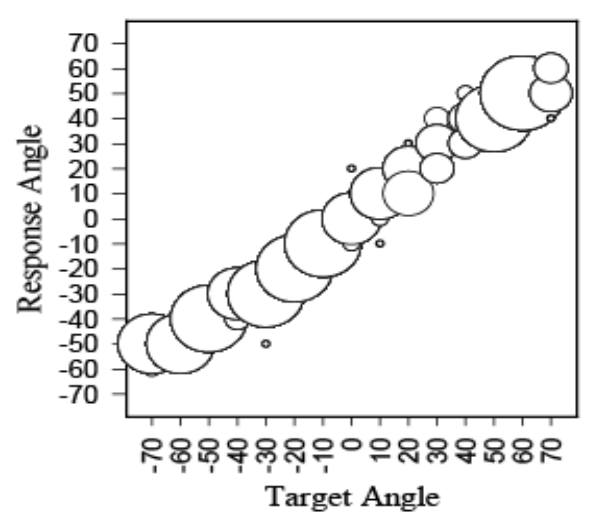

HI Unaided Subject F

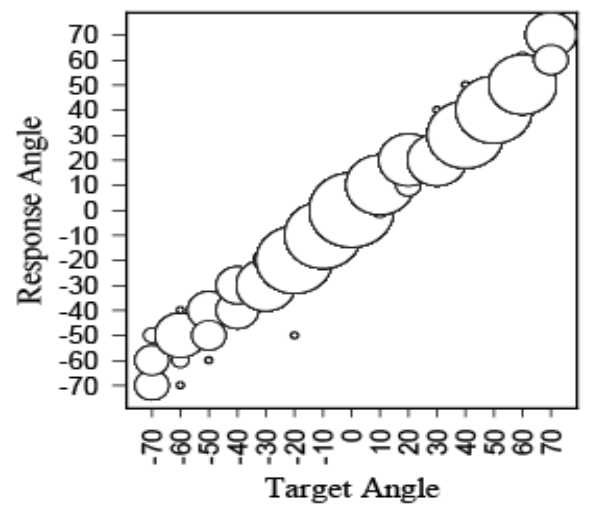

Figure B-5. Hearing Impaired Participants (A-F) unaided perceived angle plotted as a function of actual target angle of the stimuli. The bubble size indicates the number of times (out of ten trials) that a particular angle was chosen. Larger bubbles indicate an angle that was often perceived, whereas smaller bubbles indicate an angle was rarely perceived. 
HI Unaided Subject G

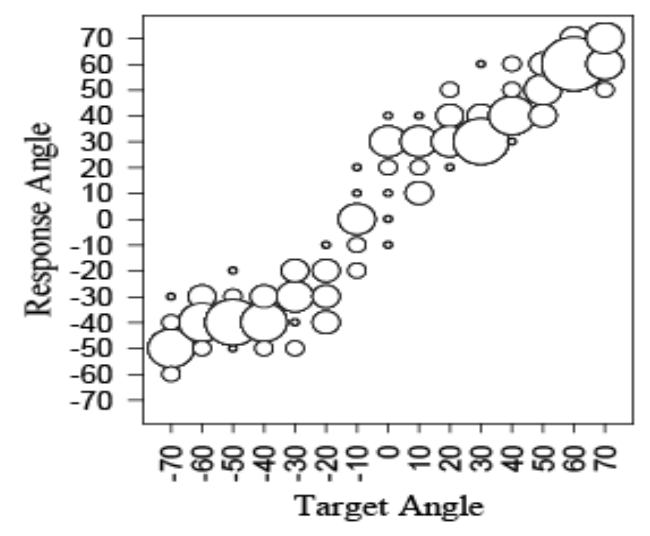

HI Unaided Subject $\mathrm{H}$

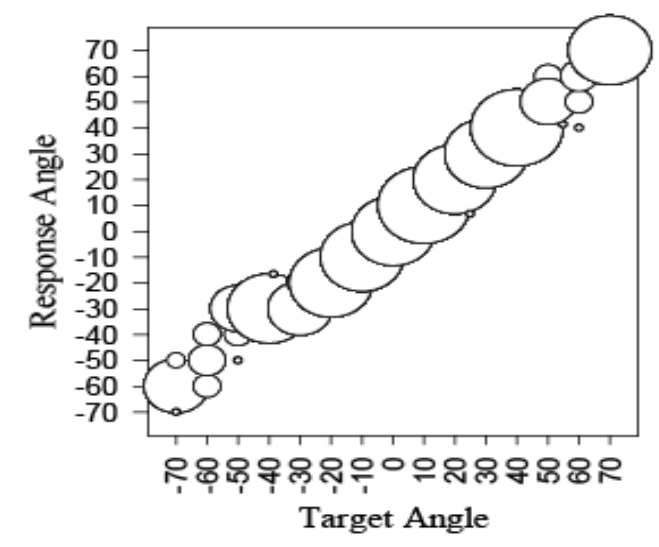

HI Unaided Subject I

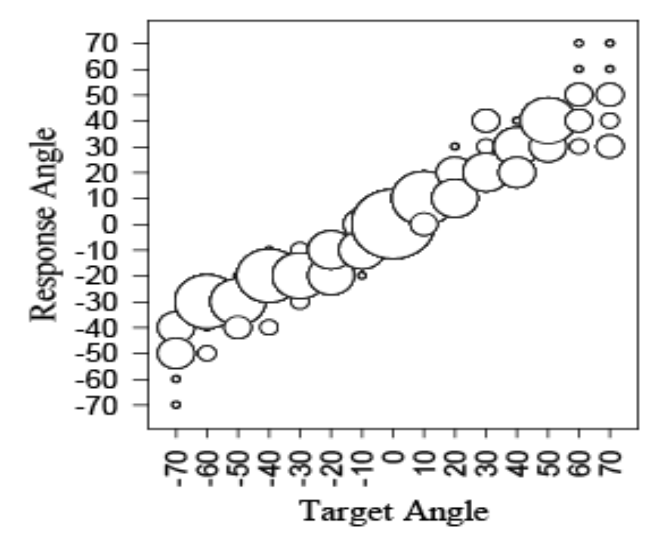

Figure B-6. Hearing Impaired Participants (G-I) unaided perceived angle plotted as a function of actual target angle of the stimuli. The bubble size indicates the number of times (out of ten trials) that a particular angle was chosen. Larger bubbles indicate an angle that was often perceived, whereas smaller bubbles indicate an angle was rarely perceived. 

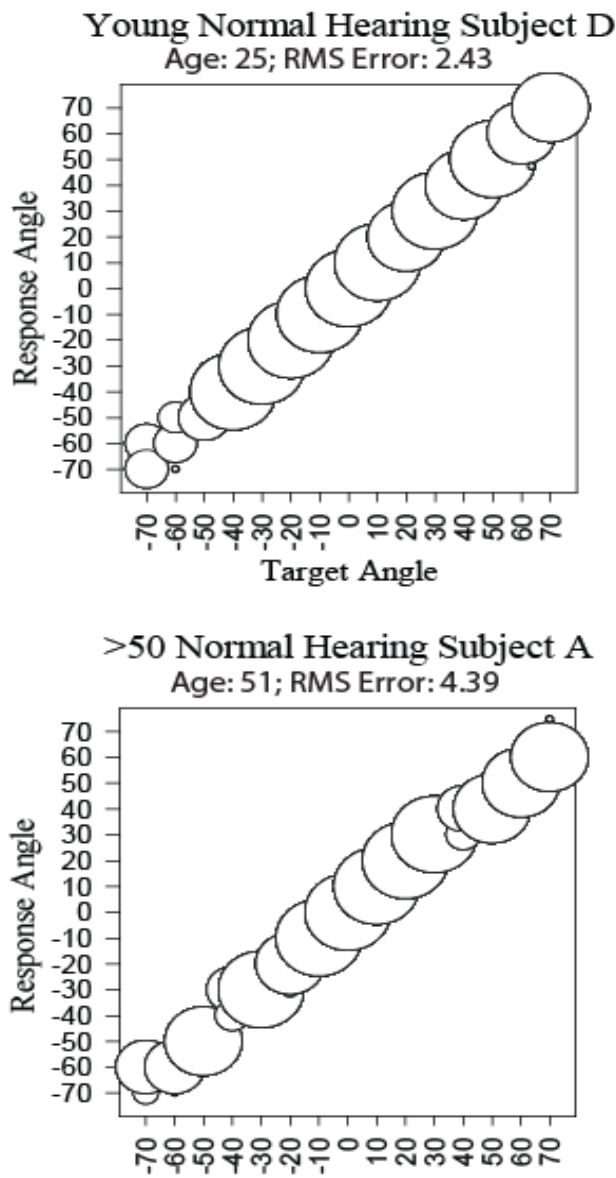

Target Angle

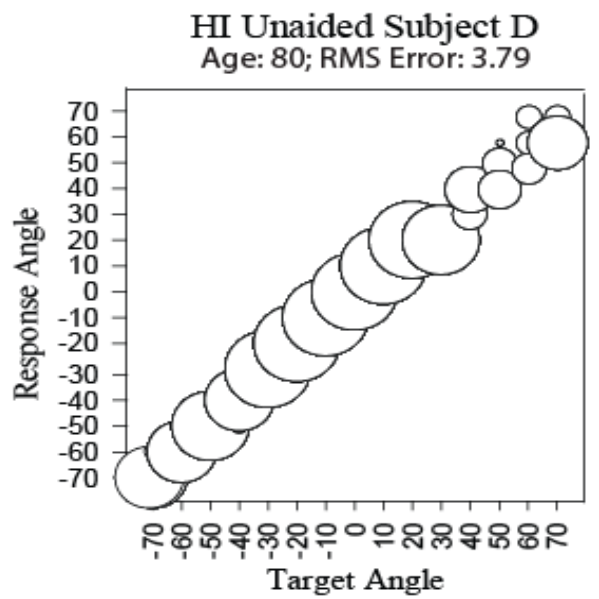

Young Normal Hearing Subject I

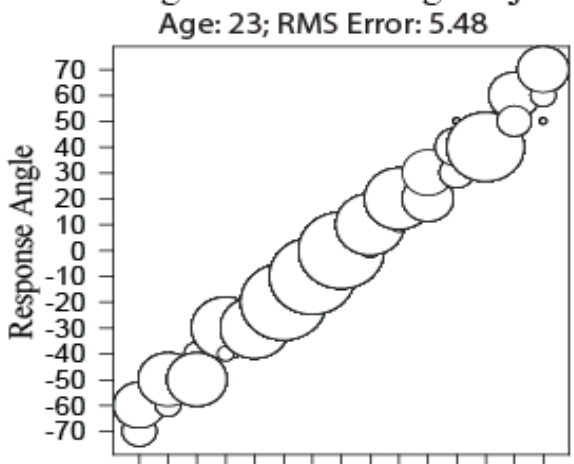

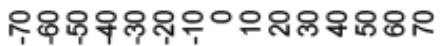

Target Angle

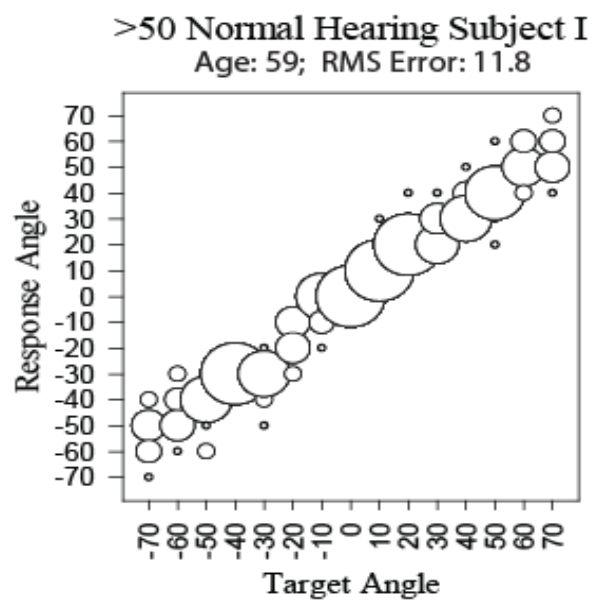

HI Unaided Subject I Age: 78; RMS Error: 14.31

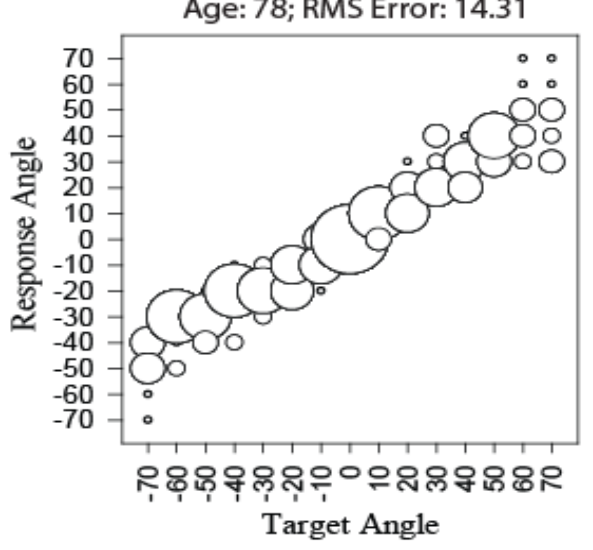

Figure B-7. Unaided best and worst perceived angle across each participant group plotted as a function of actual target angle of the stimuli. The bubble size indicates the number of times (out of ten trials) that a particular angle was chosen. Larger bubbles indicate an angle that was often perceived, whereas smaller bubbles indicate an angle was rarely perceived. 
HI Subject A; Open - Omnidirectional

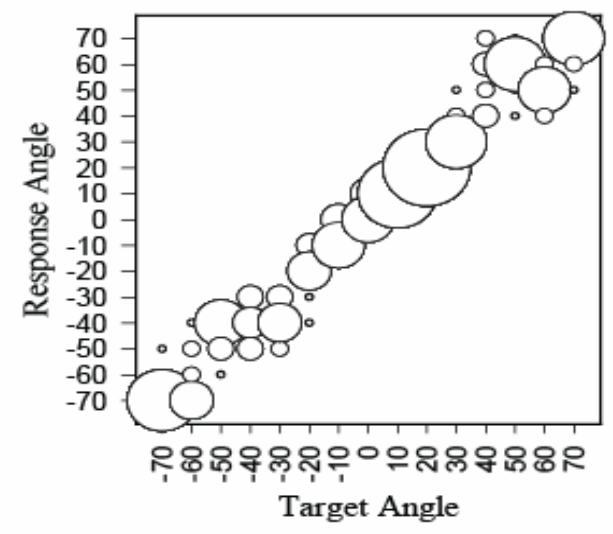

HI Subject B; Open - Omnidirectional

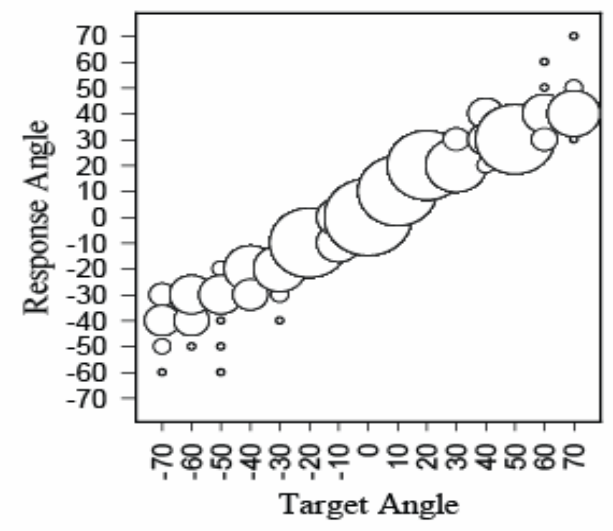

HI Subject C; Open - Omnidirectional

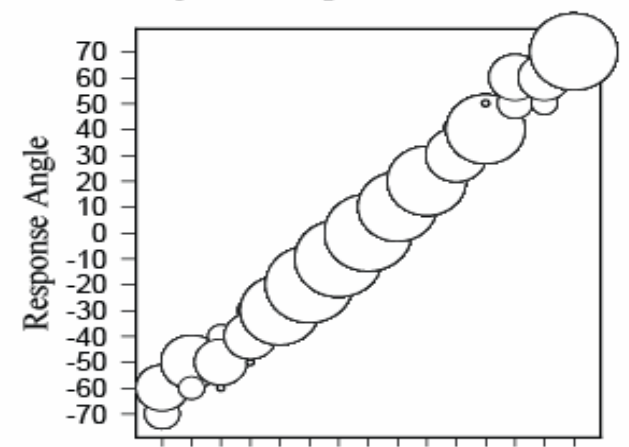

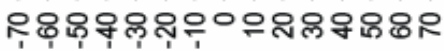

Target Angle
HI Subject D; Open - Omnidirectional

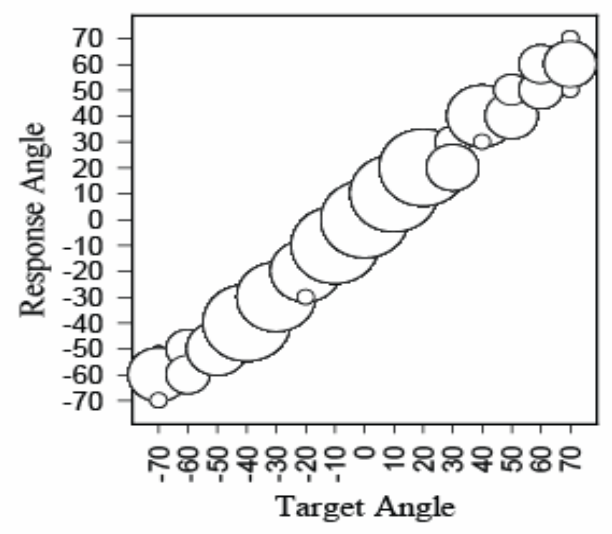

HISubject E; Open - Omnidirectional

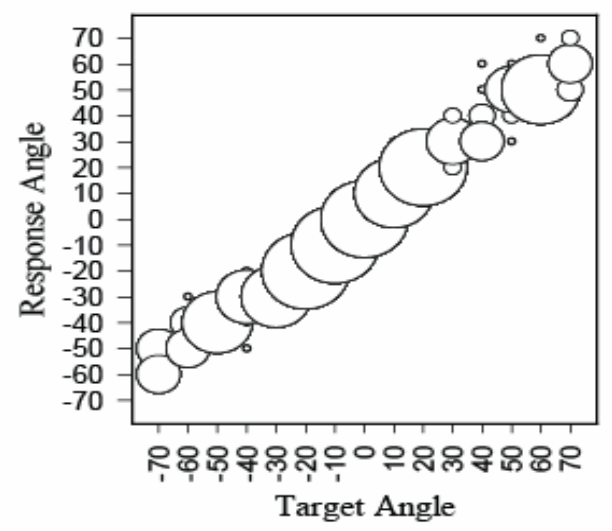

HI Subject F; Open - Omnidirectional

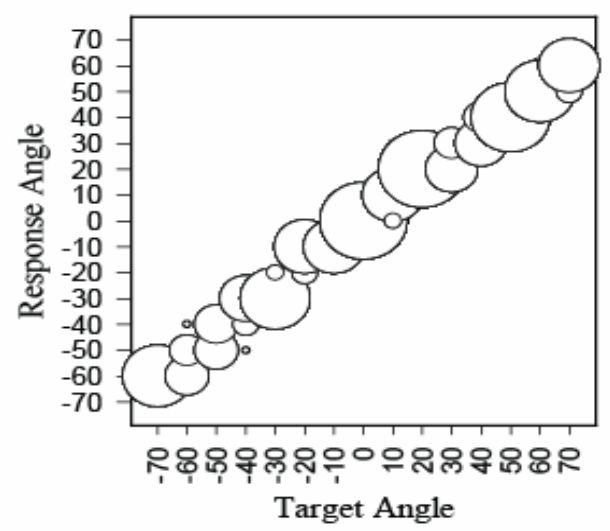

Figure B-8. Hearing Impaired Participants (A-F) Open-Omnidirectional perceived angle across each participant group plotted as a function of actual target angle of the stimuli. The bubble size indicates the number of times (out of ten trials) that a particular angle was chosen. Larger bubbles indicate an angle that was often perceived, whereas smaller bubbles indicate an angle was rarely perceived. 
HI Subject G; Open - Omnidirectional

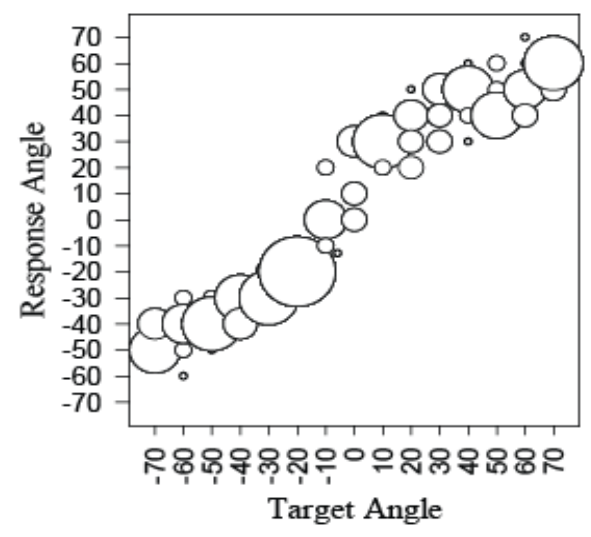

HI Subject H; Open - Omnidirectional

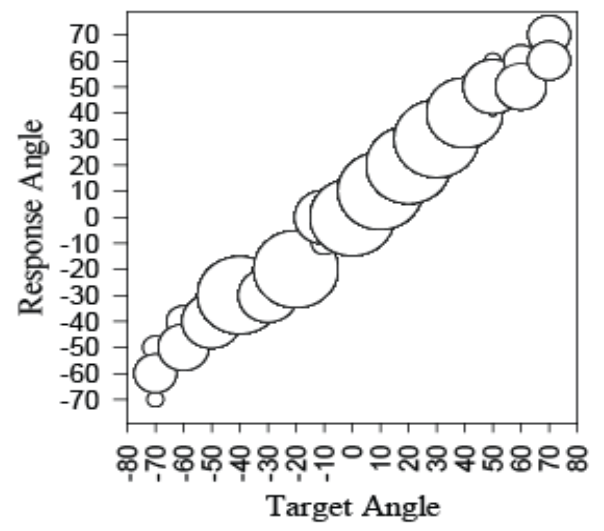

HI Subject I; Open - Omnidirectional

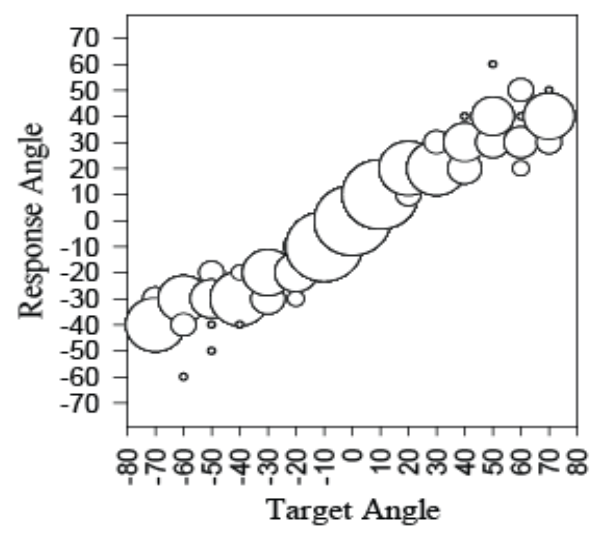

Figure B-9. Hearing Impaired Participants (G-I) Open-Omnidirectional perceived angle across each participant group plotted as a function of actual target angle of the stimuli. The bubble size indicates the number of times (out of ten trials) that a particular angle was chosen. Larger bubbles indicate an angle that was often perceived, whereas smaller bubbles indicate an angle was rarely perceived. 
HI Subject A; Open - Adaptive Directional

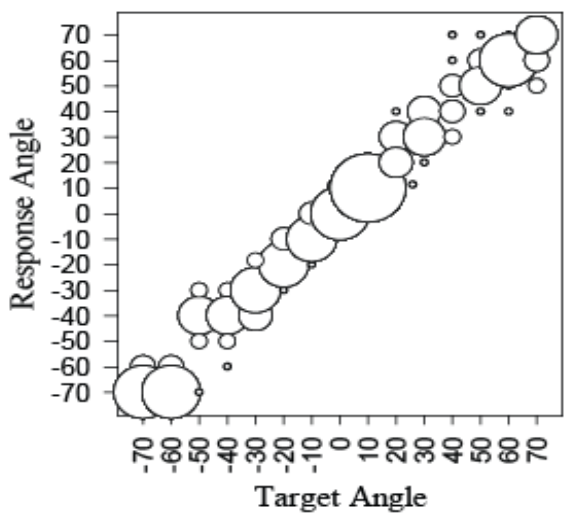

HI Subject B; Open - Adaptive Directional

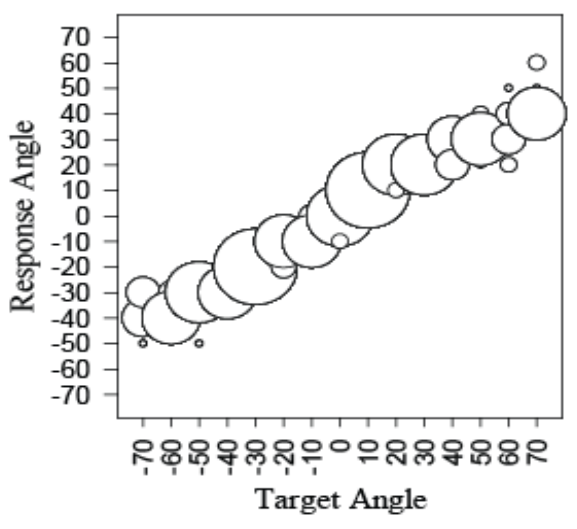

HI Subject C; Open - Adaptive Directional

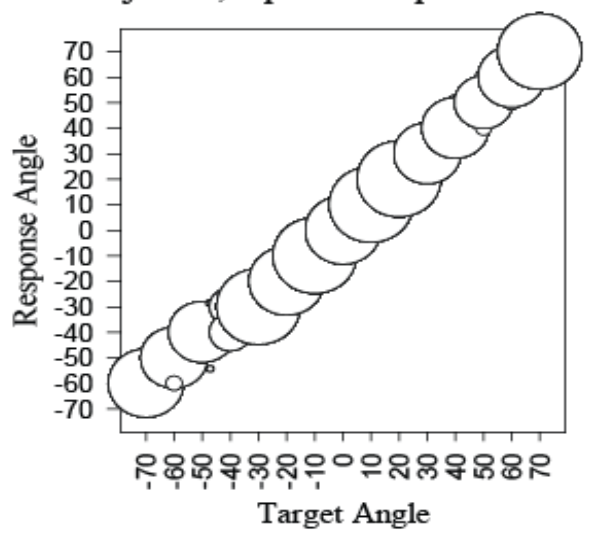

HI Subject D; Open - Adaptive Directional

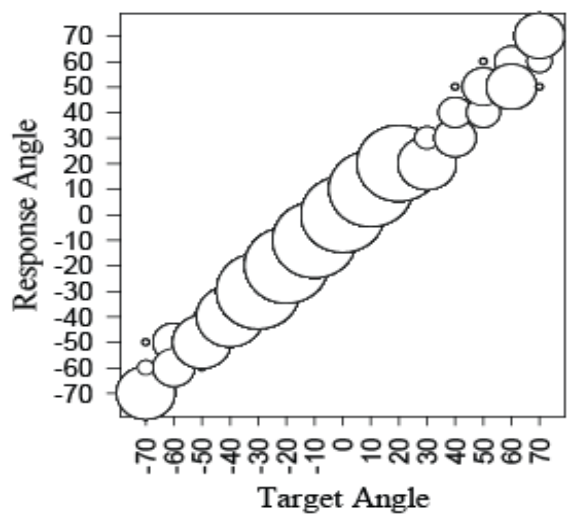

HI Subject E; Open - Adaptive Directional

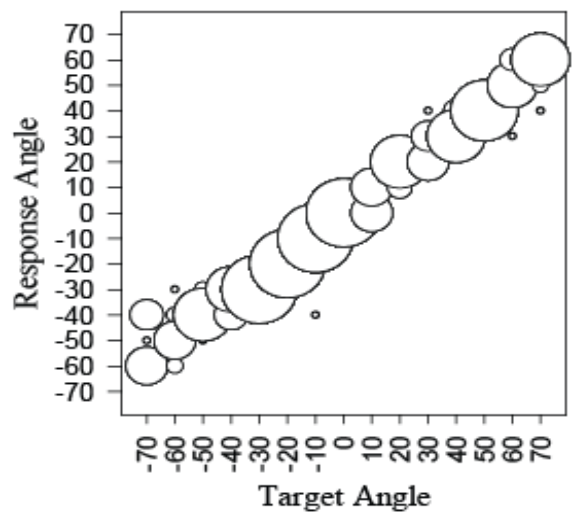

HI Subject F; Open - Adaptive Directional

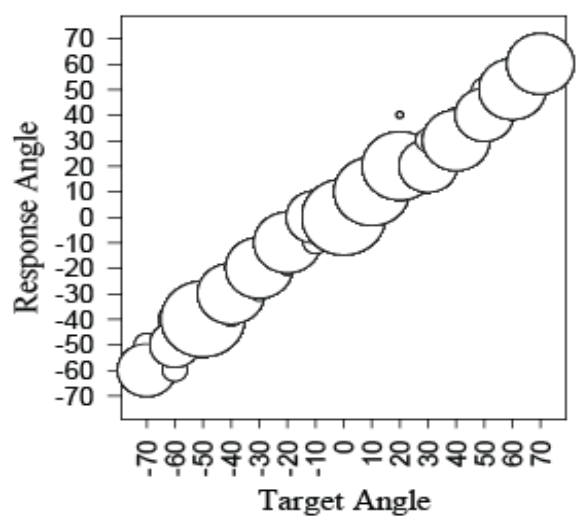

Figure B-10. Hearing Impaired Participants (A-F) Open-Adaptive Directional perceived angle across each participant group plotted as a function of actual target angle of the stimuli. The bubble size indicates the number of times (out of ten trials) that a particular angle was chosen. Larger bubbles indicate an angle that was often perceived, whereas smaller bubbles indicate an angle was rarely perceived. 
HI Subject G; Open - Adaptive Directional

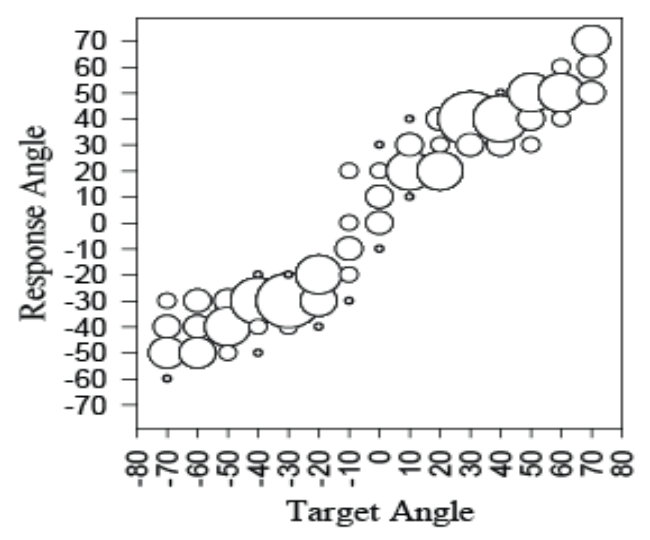

HI Subject H; Open - Adaptive Directional

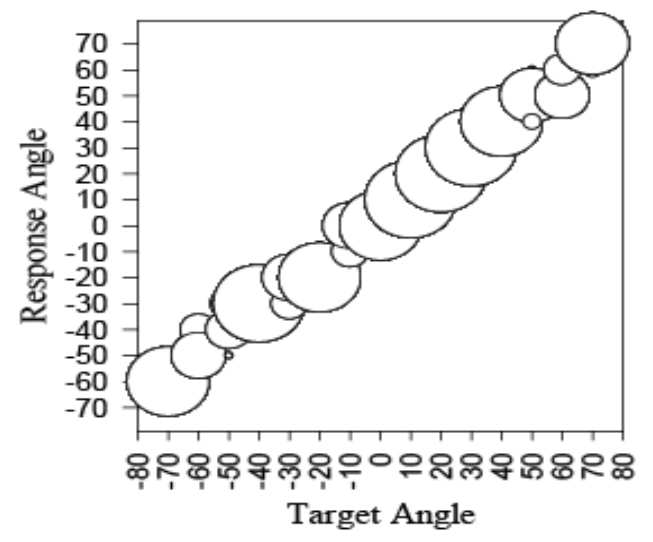

HI Subject I; Open - Adaptive Directional

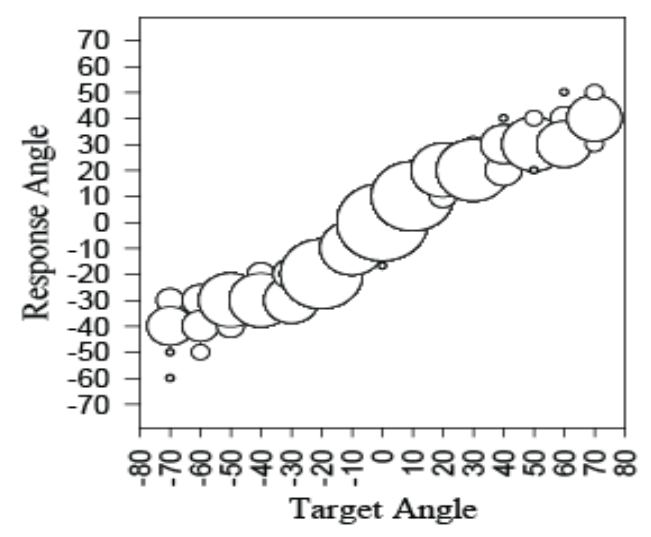

Figure B-11. Hearing Impaired Participants (G-I) Open-Adaptive Directional perceived angle across each participant group plotted as a function of actual target angle of the stimuli. The bubble size indicates the number of times (out of ten trials) that a particular angle was chosen. Larger bubbles indicate an angle that was often perceived, whereas smaller bubbles indicate an angle was rarely perceived. 
HI Subject A; Open - Fixed Directional

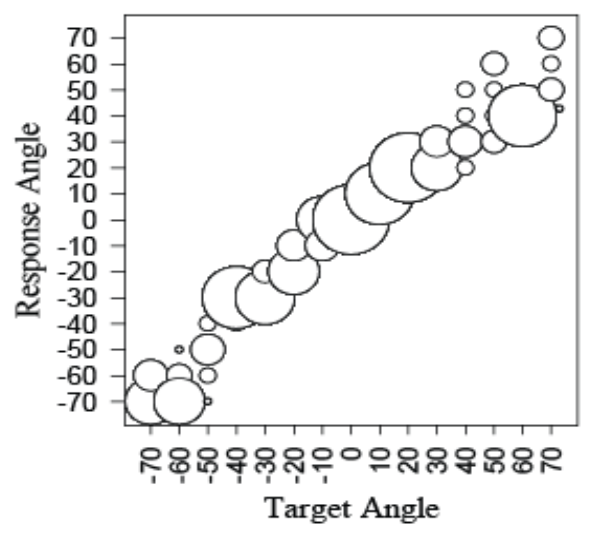

HI Subject B; Open - Fixed Directional

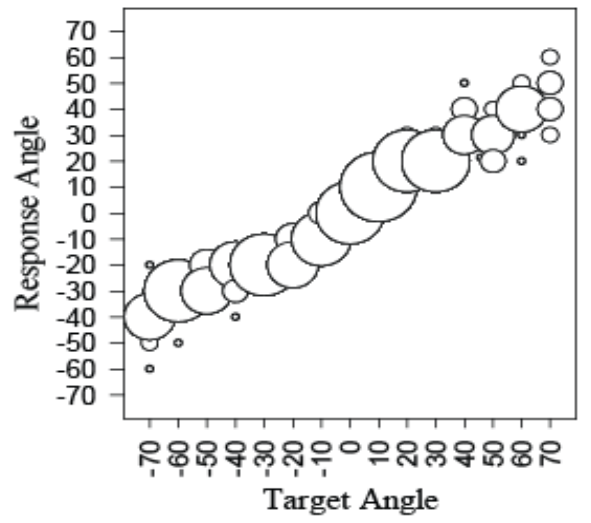

HISubject C; Open - Fixed Directional

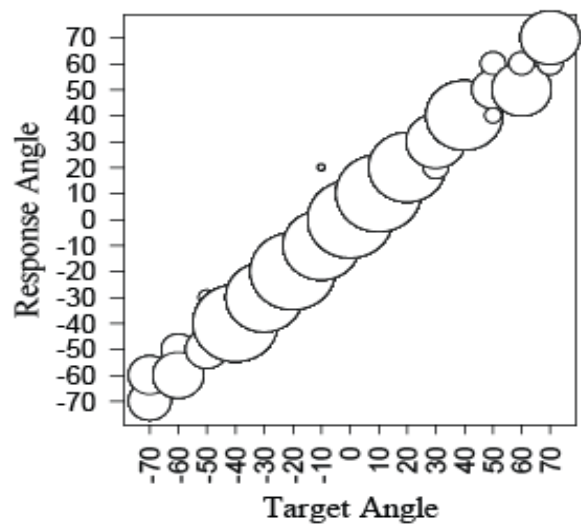

HI Subject D; Open - Fixed Directional

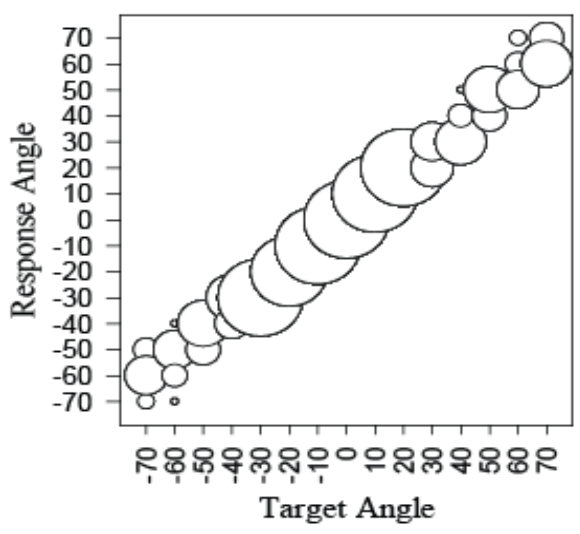

HI Subject E; Open - Fixed Directional

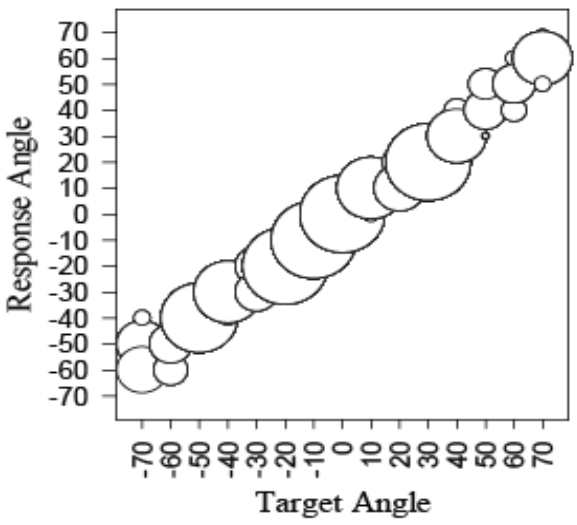

HISubject F; Open - Fixed Directional

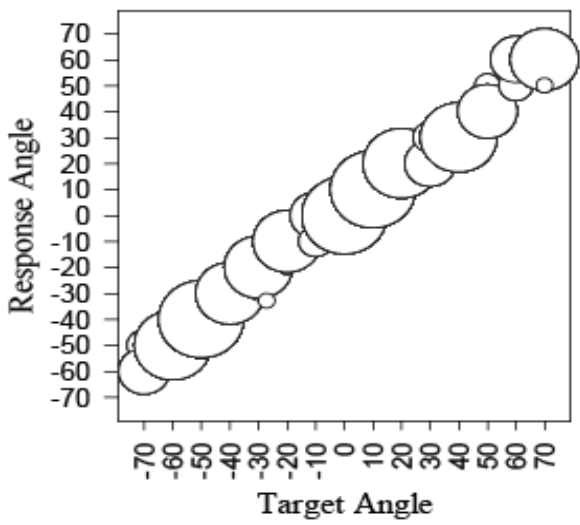

Figure B-12. Hearing Impaired Participants (A-F) Open-Fixed Directional perceived angle across each participant group plotted as a function of actual target angle of the stimuli. The bubble size indicates the number of times (out of ten trials) that a particular angle was chosen. Larger bubbles indicate an angle that was often perceived, whereas smaller bubbles indicate an angle was rarely perceived. 
HI Subject G; Open - Fixed Directional

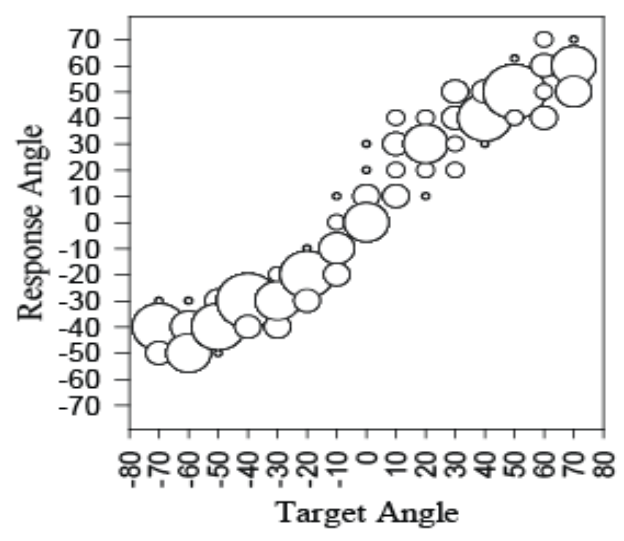

HI Subject H; Open - Fixed Directional

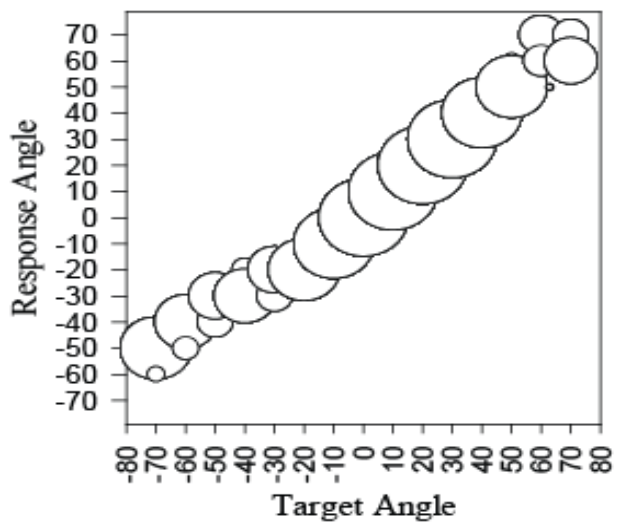

HI Subject I; Open - Fixed Directional

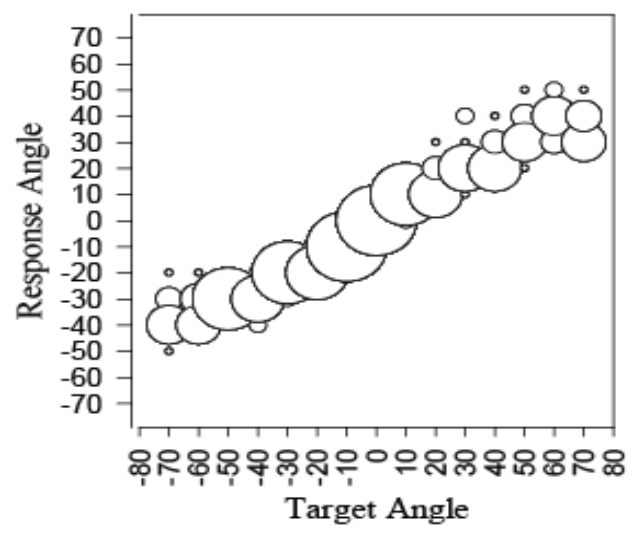

Figure B-13. Hearing Impaired Participants (G-I) Open-Fixed Directional perceived angle across each participant group plotted as a function of actual target angle of the stimuli. The bubble size indicates the number of times (out of ten trials) that a particular angle was chosen. Larger bubbles indicate an angle that was often perceived, whereas smaller bubbles indicate an angle was rarely perceived. 
HI Subject A; Micro - Omnidirectional

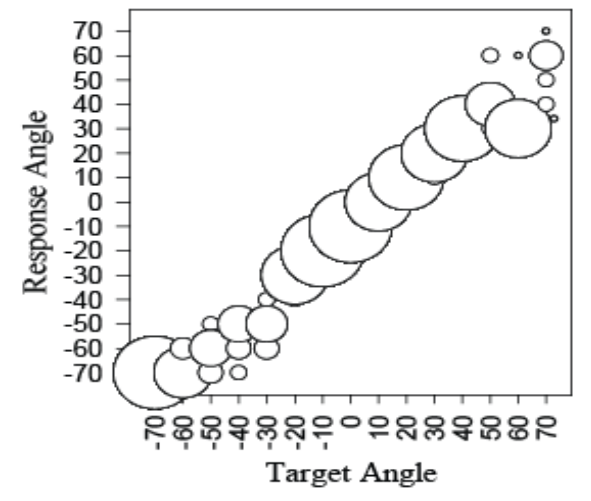

HI Subject B; Micro - Omnidirectional

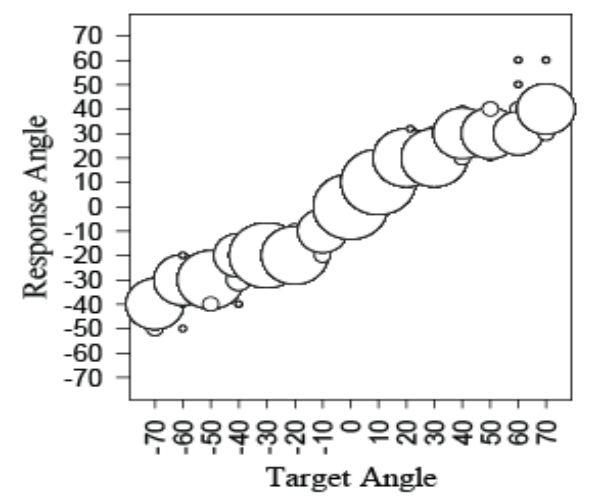

HI Subject C; Micro- Omnidirectional

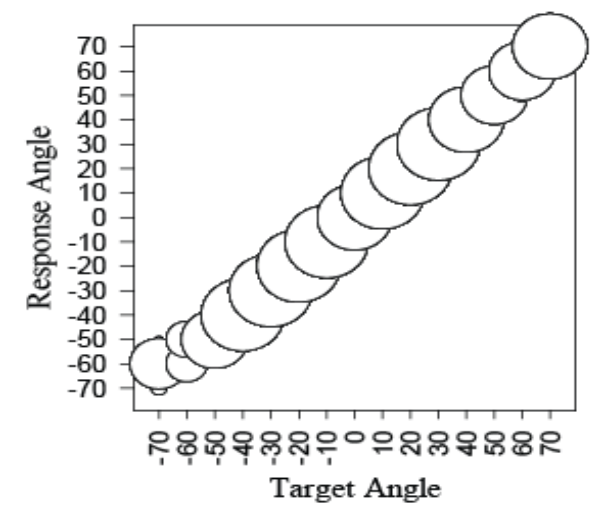

HI Subject D; Micro - Omnidirectional

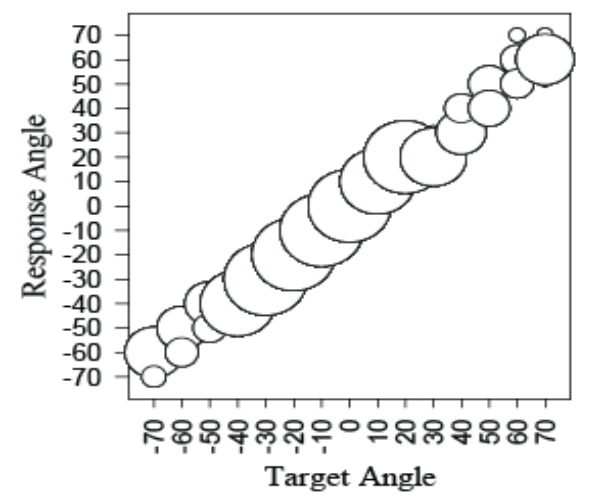

HI Subject E; Micro - Omnidirectional

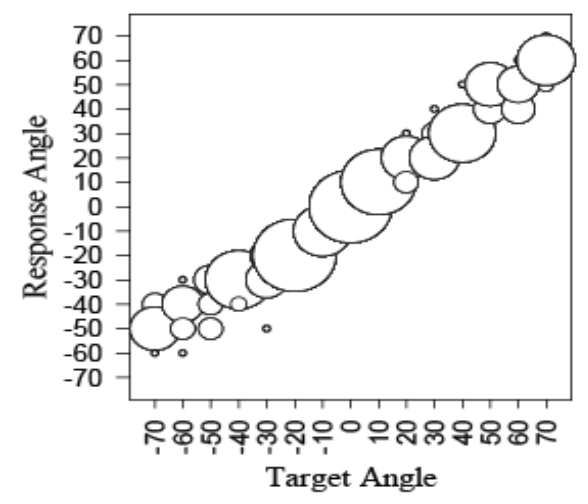

HI Subject F; Micro - Omnidirectional

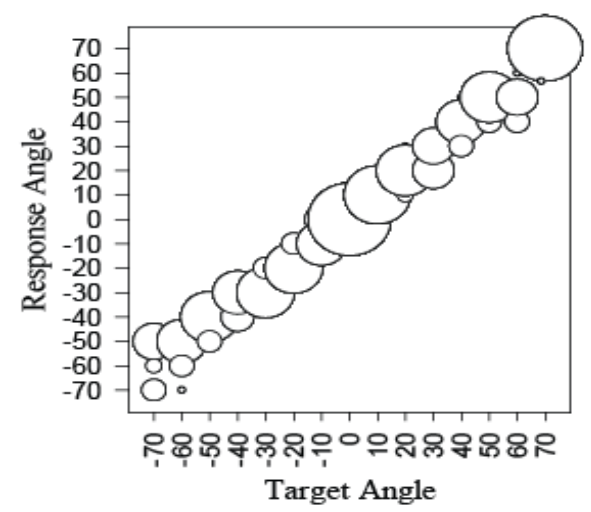

Figure B-14. Hearing Impaired Participants (A-F) Micromold-Omnidirectional perceived angle across each participant group plotted as a function of actual target angle of the stimuli. The bubble size indicates the number of times (out of ten trials) that a particular angle was chosen. Larger bubbles indicate an angle that was often perceived, whereas smaller bubbles indicate an angle was rarely perceived. 
HI Subject G; Micro - Omnidirectional

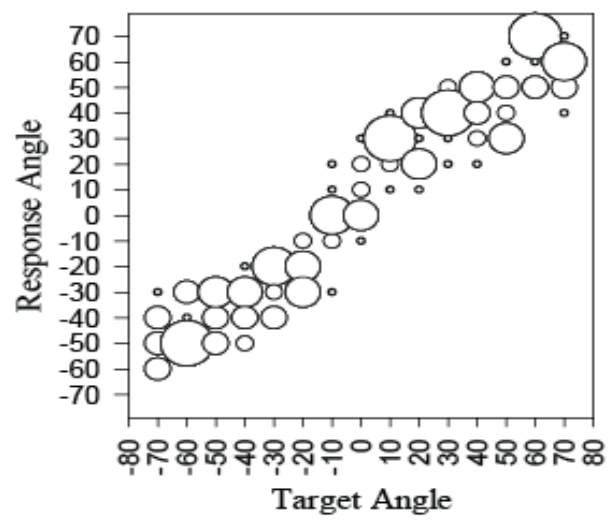

HI Subject H; Micro - Omnidirectional

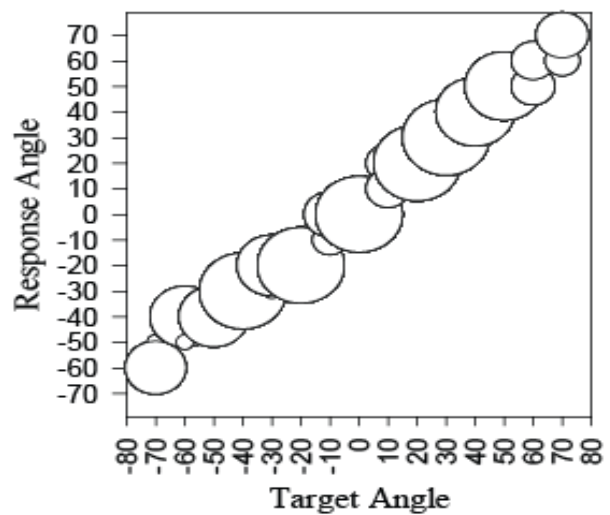

HI Subject I; Micro - Omnidirectional

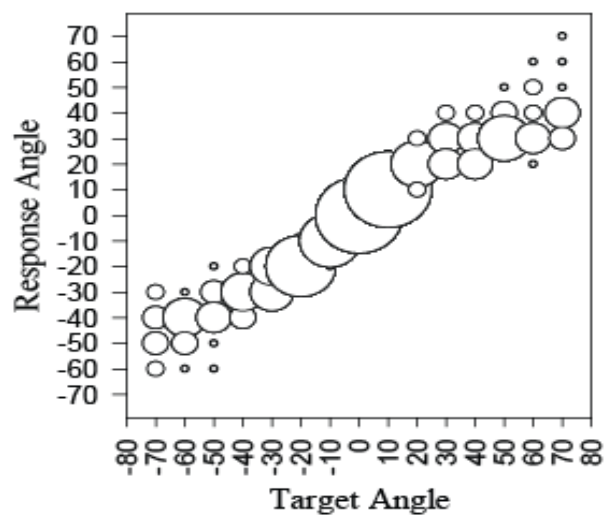

Figure B-15. Hearing Impaired Participants (G-I) Micromold-Omnidirectional perceived angle across each participant group plotted as a function of actual target angle of the stimuli. The bubble size indicates the number of times (out of ten trials) that a particular angle was chosen. Larger bubbles indicate an angle that was often perceived, whereas smaller bubbles indicate an angle was rarely perceived. 
HI Subject A; Micro - Adaptive Directional

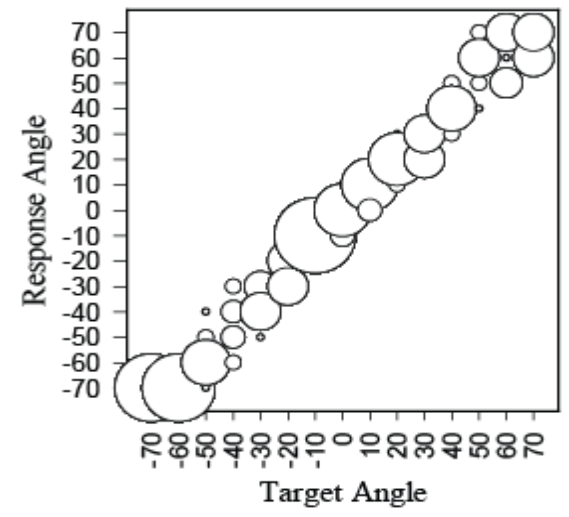

HI Subject B; Micro - Adaptive Directional

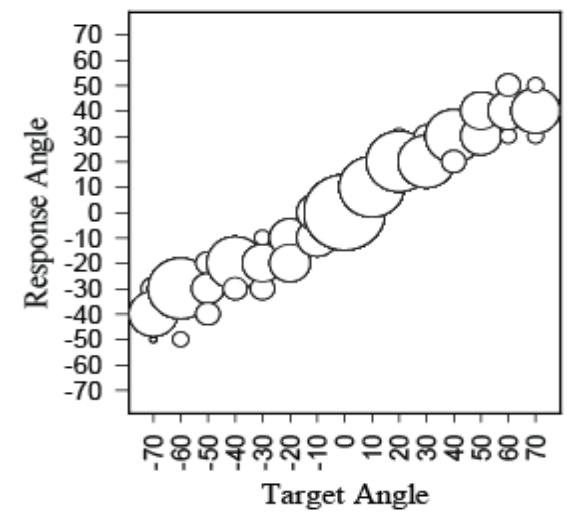

HI Subject C; Micro - Adaptive Directional

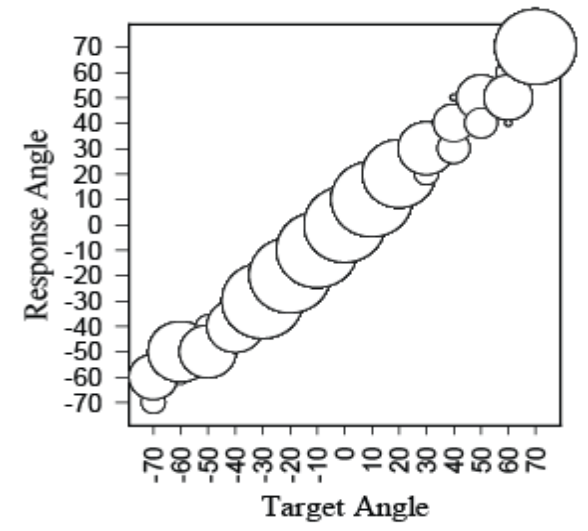

HI Subject D; Micro - Adaptive Directional

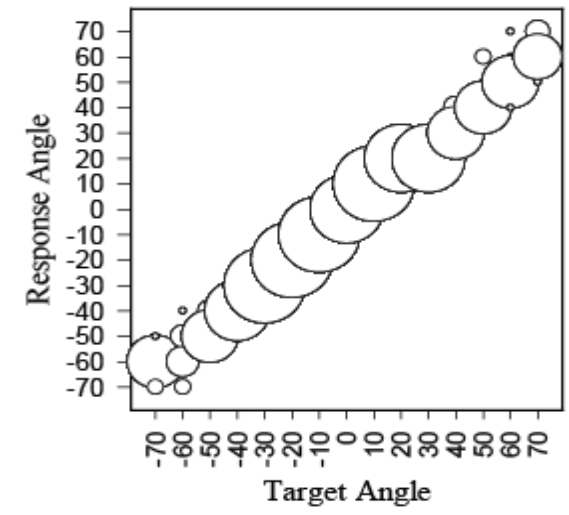

HI Subject E; Micro - Adaptive Directional

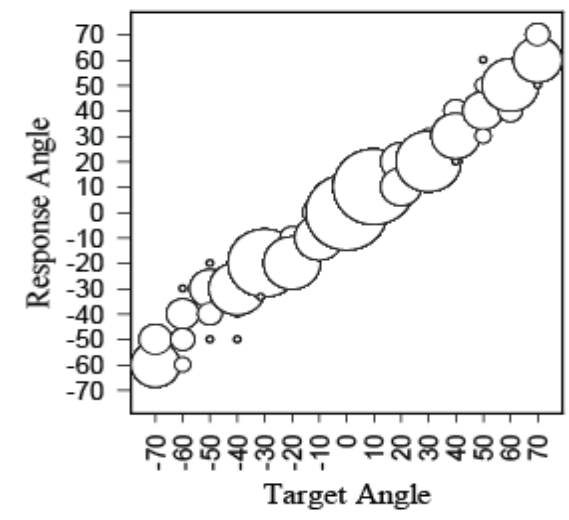

HI Subject F; Micro - Adaptive Directional

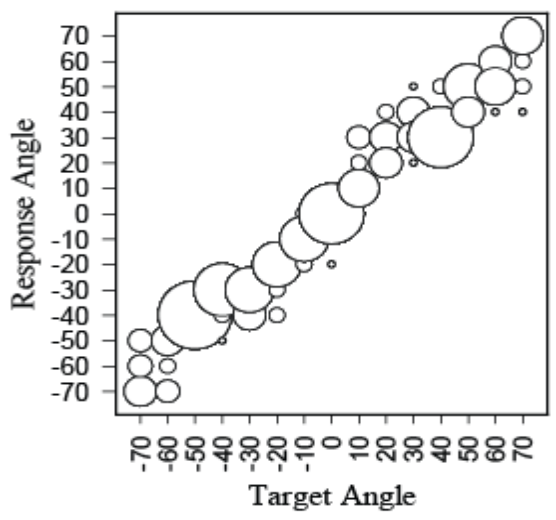

Figure B-16. Hearing Impaired Participants (A-F) Micromold-Adaptive Directional perceived angle across each participant group plotted as a function of actual target angle of the stimuli. The bubble size indicates the number of times (out of ten trials) that a particular angle was chosen. Larger bubbles indicate an angle that was often perceived, whereas smaller bubbles indicate an angle was rarely perceived. 
HI Subject G; Micro - Adaptive Directional

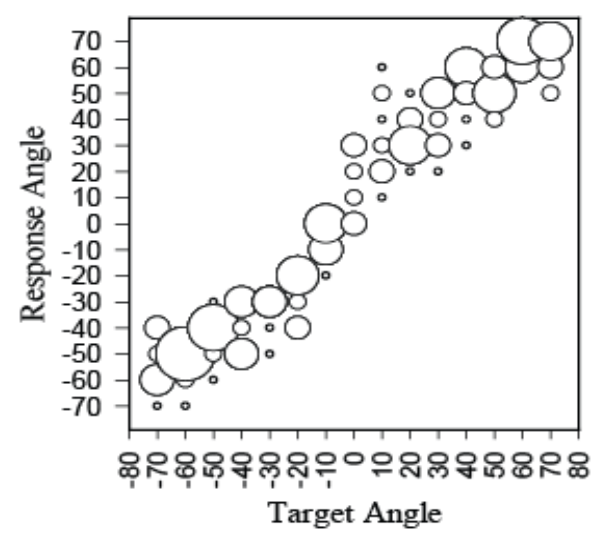

HI Subject H; Micro - Adaptive Directional

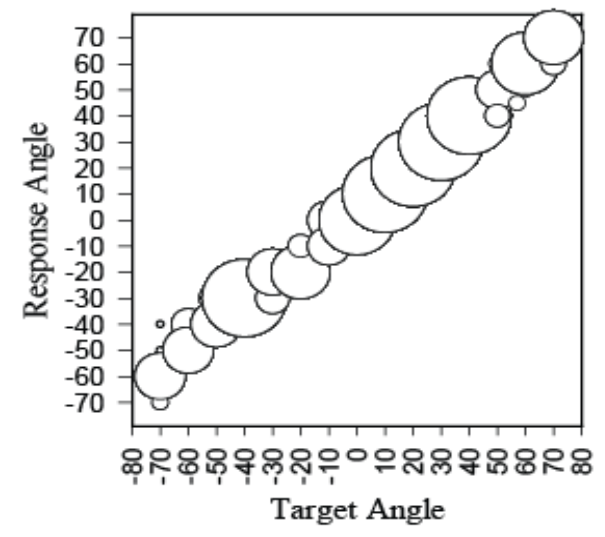

HI Subject I; Micro - Adaptive Directional

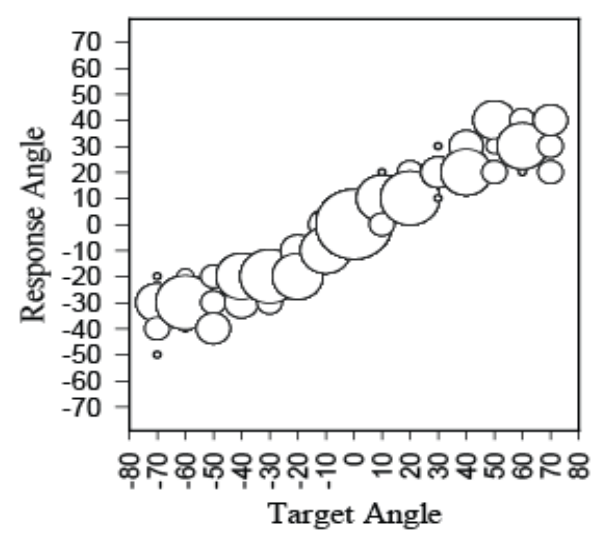

Figure B-17. Hearing Impaired Participants (G-I) Micromold-Adaptive Directional perceived angle across each participant group plotted as a function of actual target angle of the stimuli. The bubble size indicates the number of times (out of ten trials) that a particular angle was chosen. Larger bubbles indicate an angle that was often perceived, whereas smaller bubbles indicate an angle was rarely perceived. 
HI Subject A; Micro - Fixed Directional

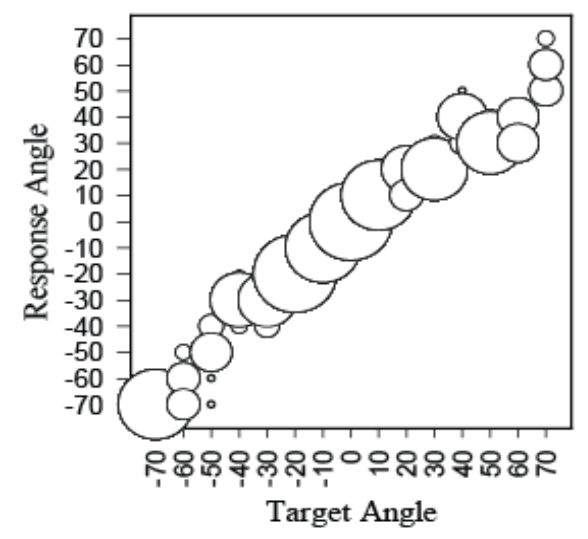

HI Subject B; Micro - Fixed Directional

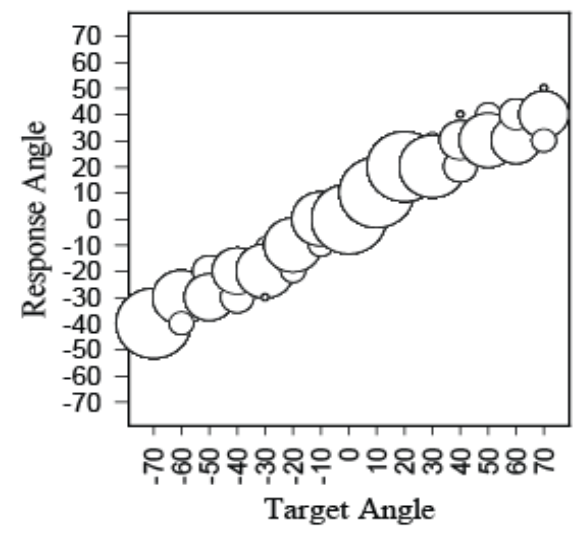

HI Subject C; Micro - Fixed Directional

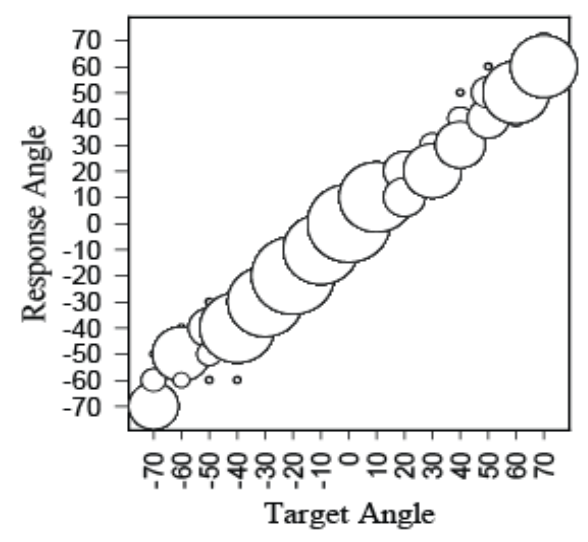

HI Subject D; Micro - Fixed Directional

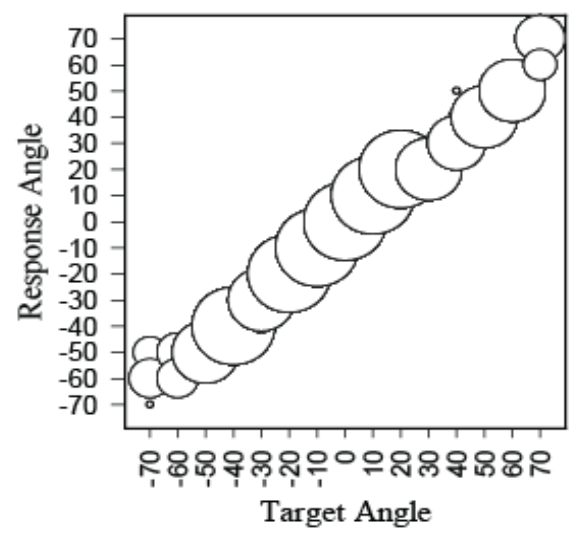

HI Subject E; Micro - Fixed Directional

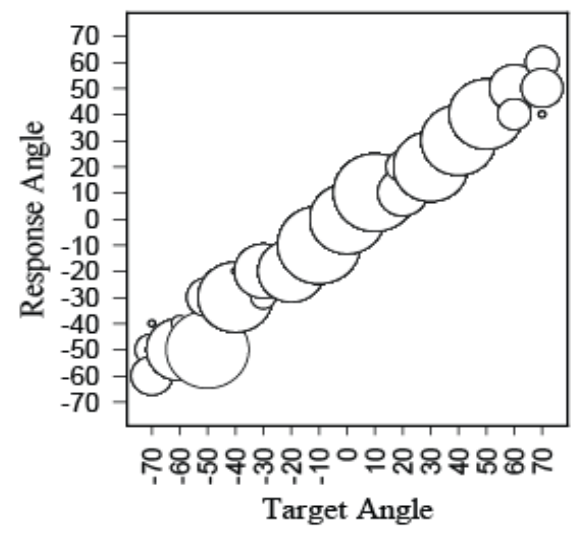

HI Subject F; Micro - Fixed Directional

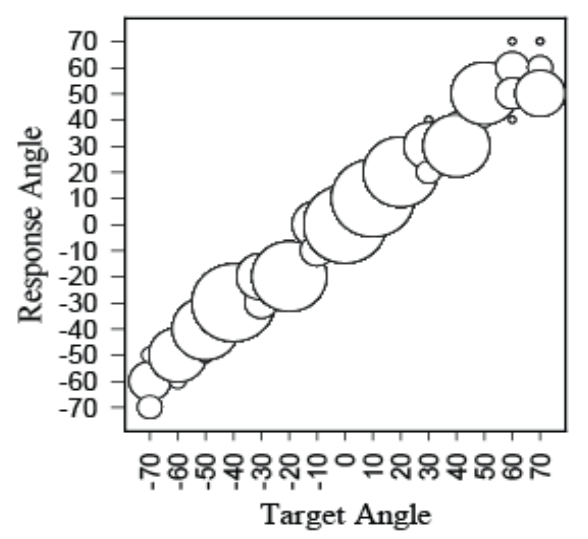

Figure B-18. Hearing Impaired Participants (A-F) Micromold-Fixed Directional perceived angle across each participant group plotted as a function of actual target angle of the stimuli. The bubble size indicates the number of times (out of ten trials) that a particular angle was chosen. Larger bubbles indicate an angle that was often perceived, whereas smaller bubbles indicate an angle was rarely perceived. 
HI Subject G; Fixed Directional

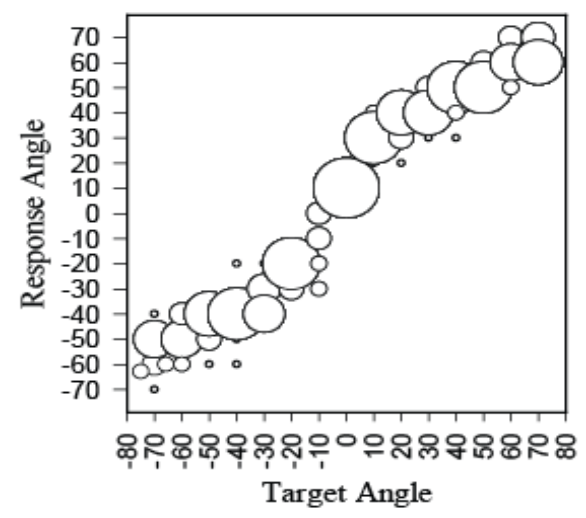

HI Subject H; Micro - Fixed Directional

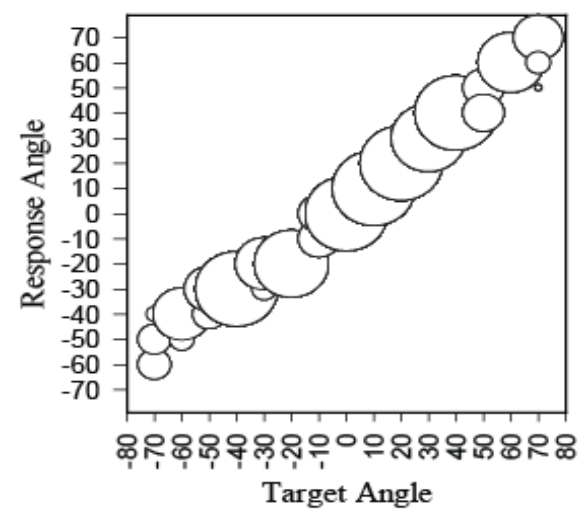

HI Subject H; Micro - Fixed Directional

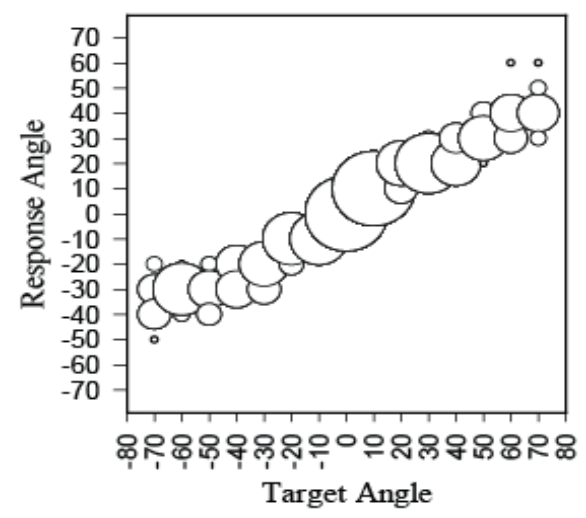

Figure B-19. Hearing Impaired Participants (G-I) Micromold-Fixed Directional perceived angle across each participant group plotted as a function of actual target angle of the stimuli. The bubble size indicates the number of times (out of ten trials) that a particular angle was chosen. Larger bubbles indicate an angle that was often perceived, whereas smaller bubbles indicate an angle was rarely perceived. 
HI Subject A; Open - Omnidirectional

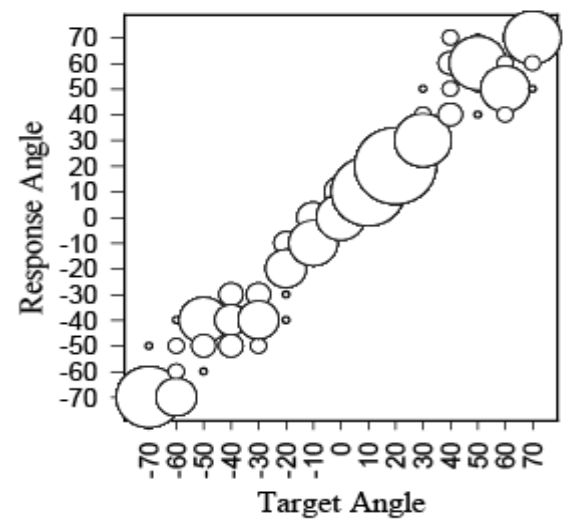

HI Subject A; Open - Adaptive Directional

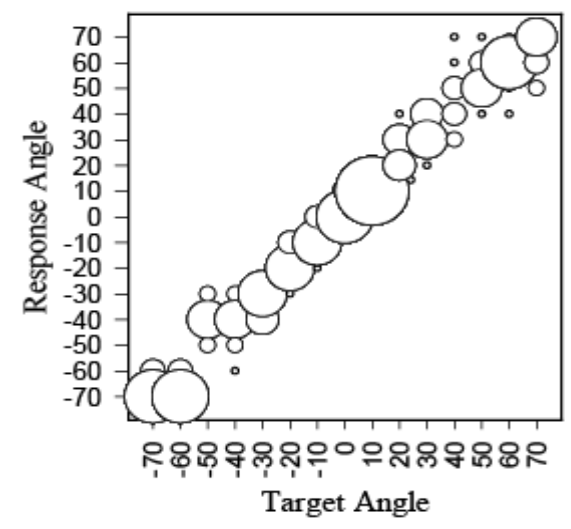

HI Subject A; Open - Fixed Directional

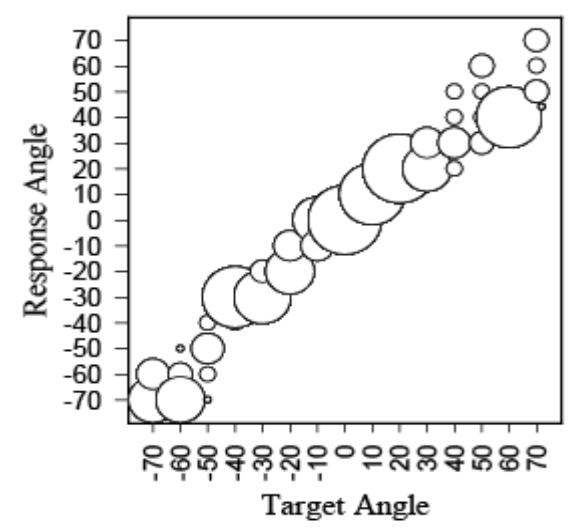

HI Subject A; Micro - Omnidirectional

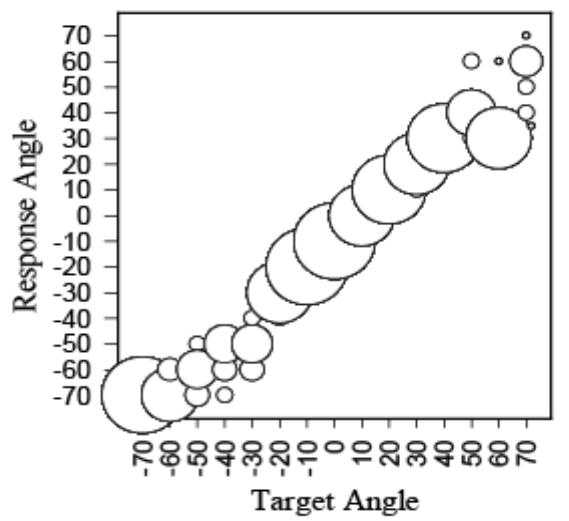

HI Subject A; Micro - Adaptive Directional

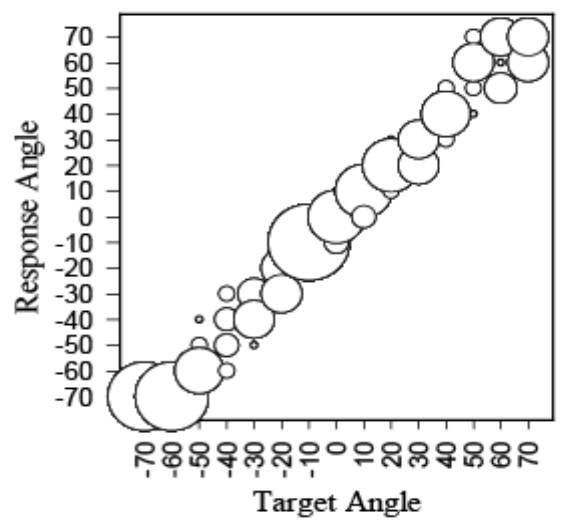

HI Subject A; Micro - Fixed Directional

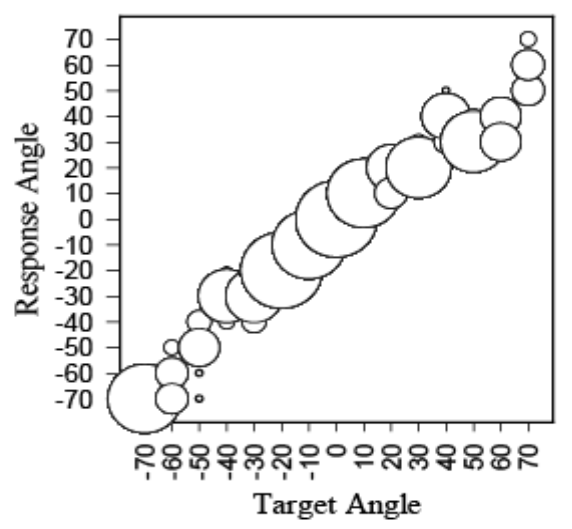

Figure B-20. Hearing Impaired Subject A perceived angle across each hearing aid condition plotted as a function of actual target angle of the stimuli. The bubble size indicates the number of times (out of ten trials) that a particular angle was chosen. Larger bubbles indicate an angle that was often perceived, whereas smaller bubbles indicate an angle was rarely perceived. 
HI Subject B; Open - Omnidirectional

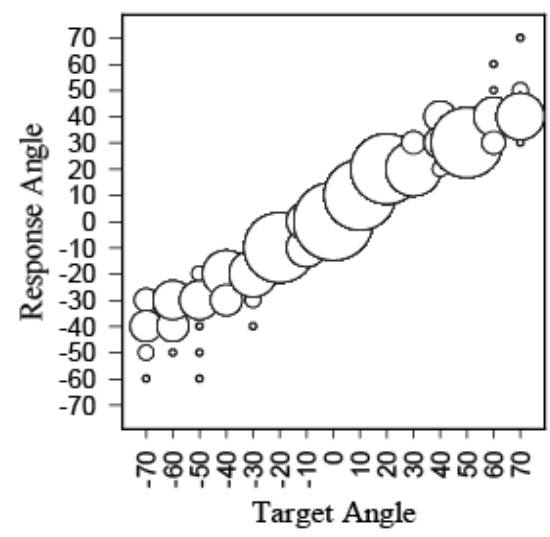

HI Subject B; Open - Adaptive Directional

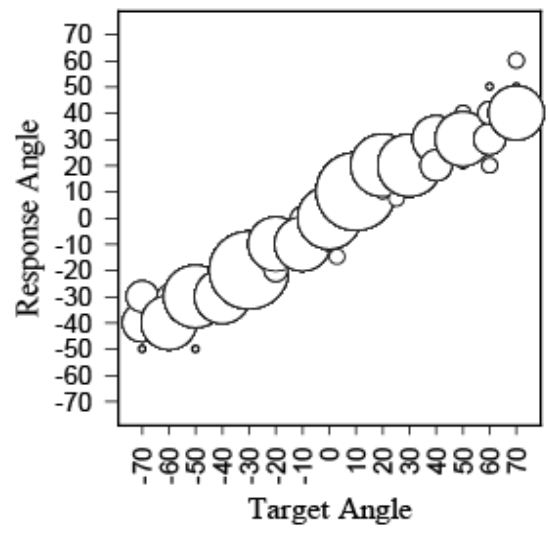

HI Subject B; Open - Fixed Directional

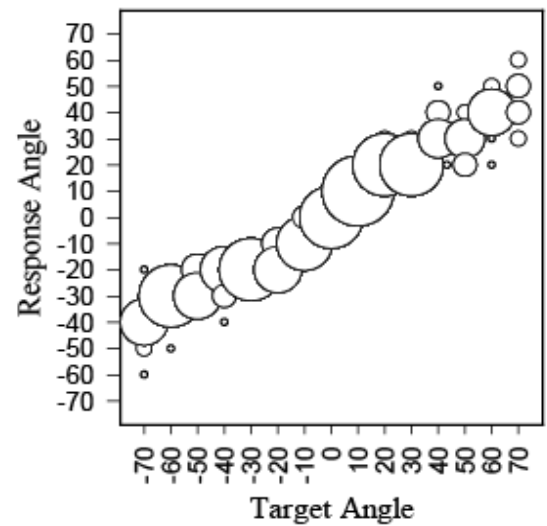

HI Subject B; Micro - Omnidirectional

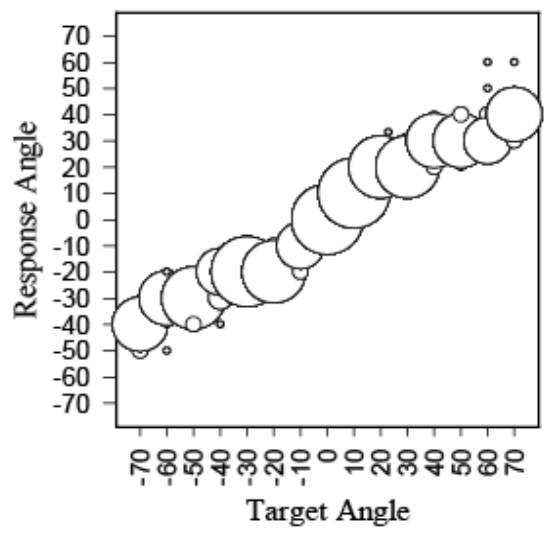

HI Subject B; Micro - Adaptive Directional

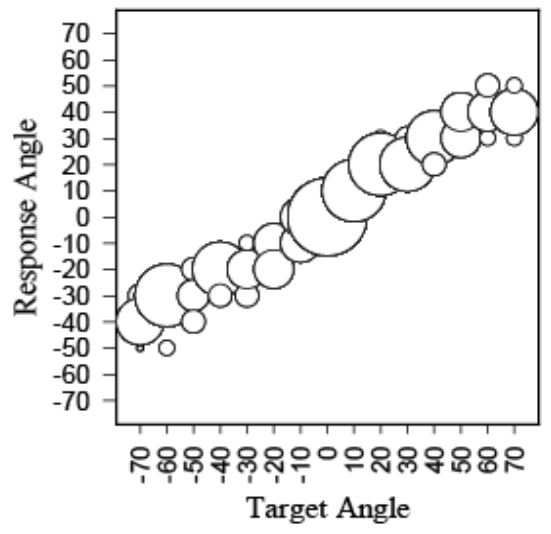

HI Subject B; Micro - Fixed Directional

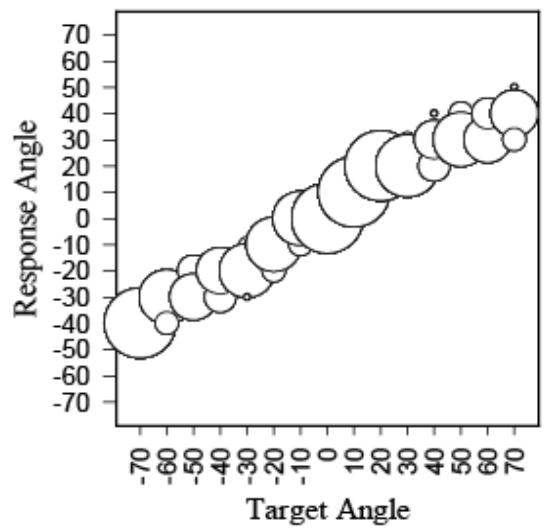

Figure B-21. Hearing Impaired Subject B perceived angle across each hearing aid condition plotted as a function of actual target angle of the stimuli. The bubble size indicates the number of times (out of ten trials) that a particular angle was chosen. Larger bubbles indicate an angle that was often perceived, whereas smaller bubbles indicate an angle was rarely perceived. 
HI Subject C; Open - Omnidirectional

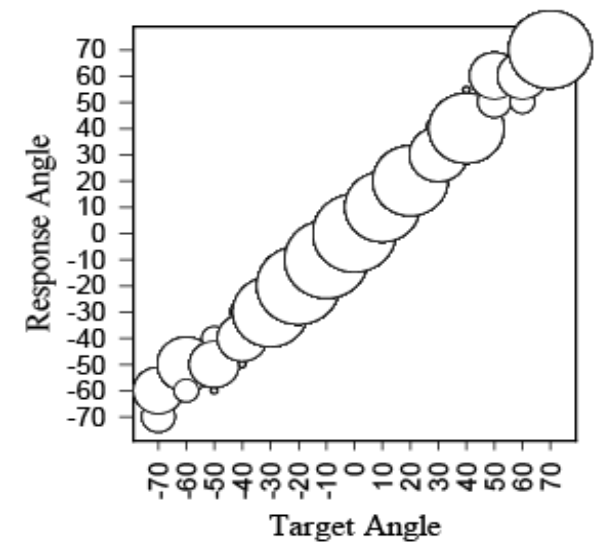

HI Subject C; Open - Adaptive Directional

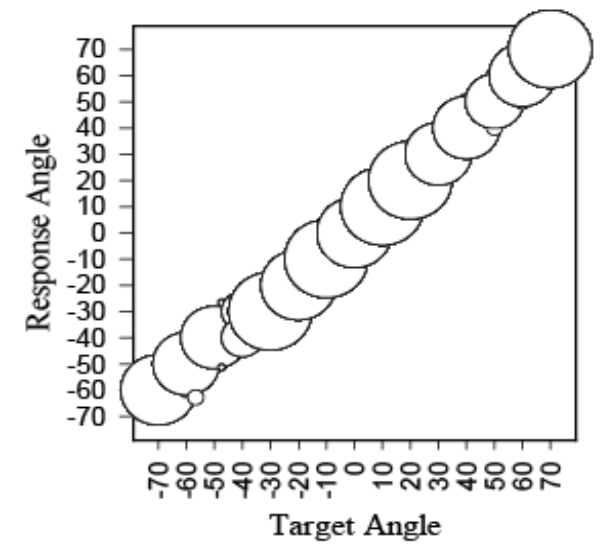

HISubject C; Open - Fixed Directional

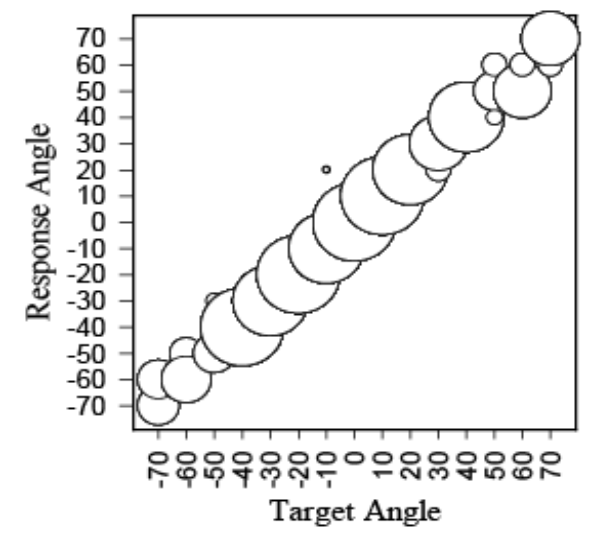

HI Subject C; Micro- Omnidirectional

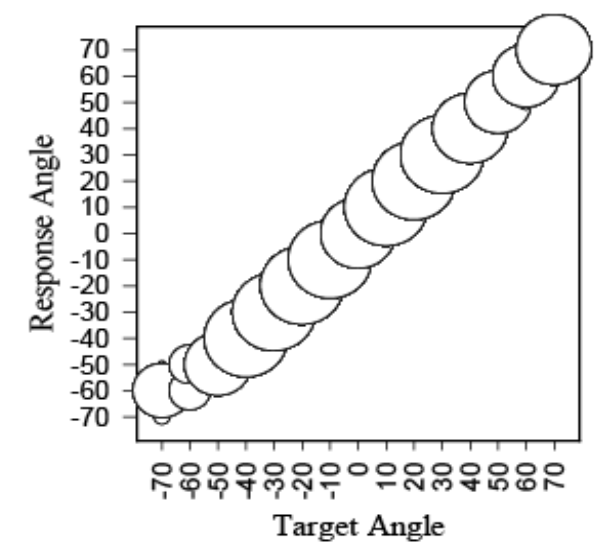

HI Subject C; Micro - Adaptive Directional

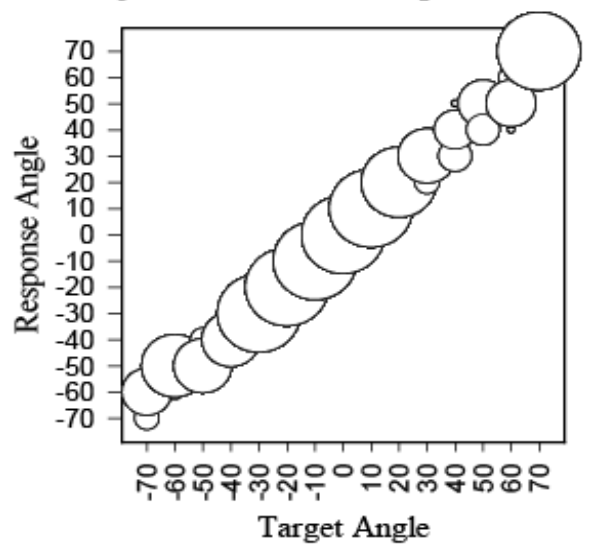

HI Subject C; Micro - Fixed Directional

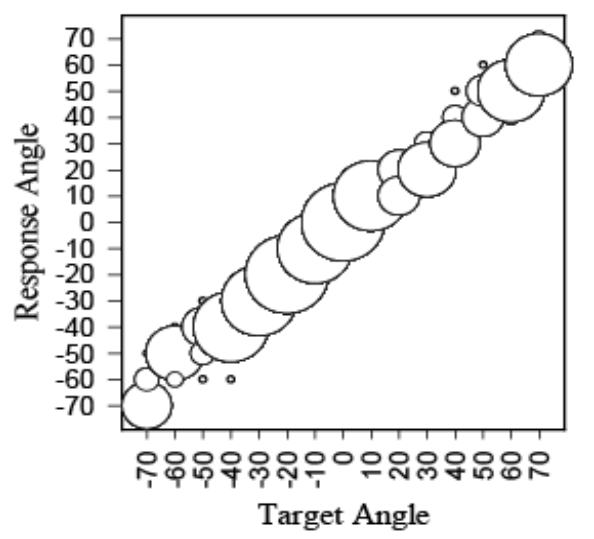

Figure B-22. Hearing Impaired Subject $C$ perceived angle across each hearing aid condition plotted as a function of actual target angle of the stimuli. The bubble size indicates the number of times (out of ten trials) that a particular angle was chosen. Larger bubbles indicate an angle that was often perceived, whereas smaller bubbles indicate an angle was rarely perceived. 
HI Subject D; Open - Omnidirectional

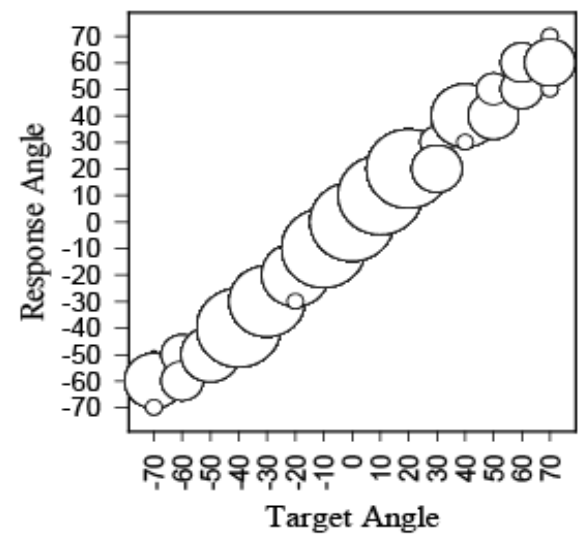

HI Subject D; Open - Adaptive Directional

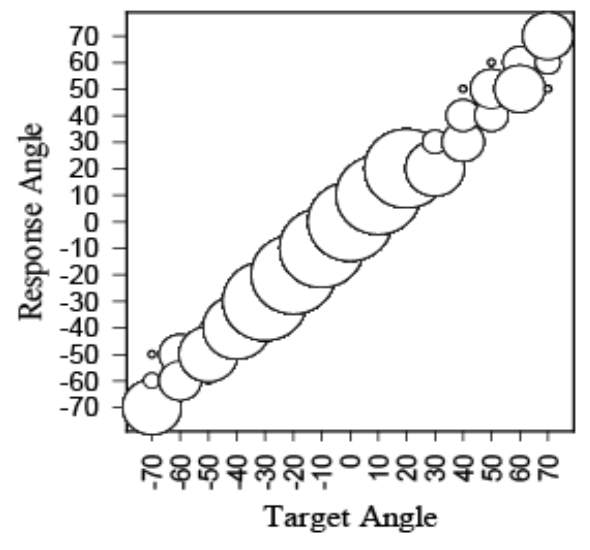

HI Subject D; Open - Fixed Directional

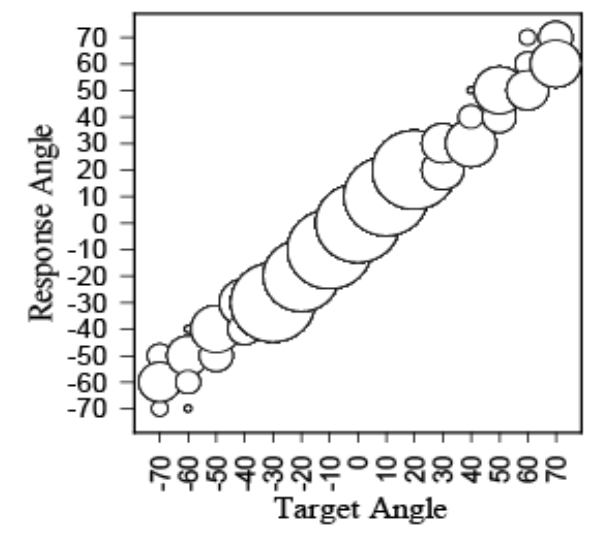

HI Subject D; Micro - Omnidirectional

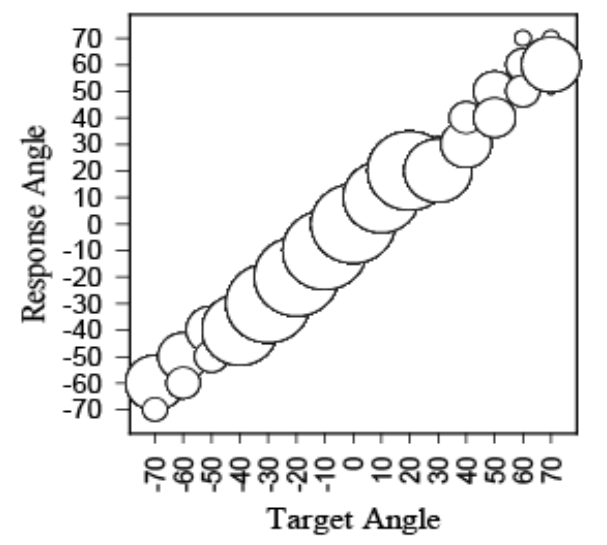

HI Subject D; Micro - Adaptive Directional

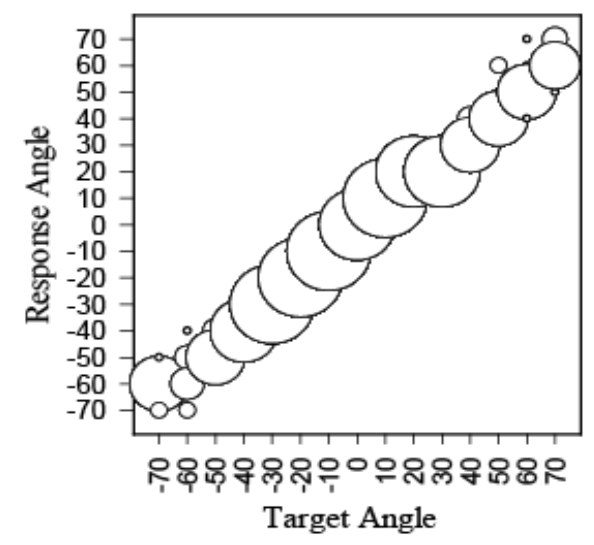

HI Subject D; Micro - Fixed Directional

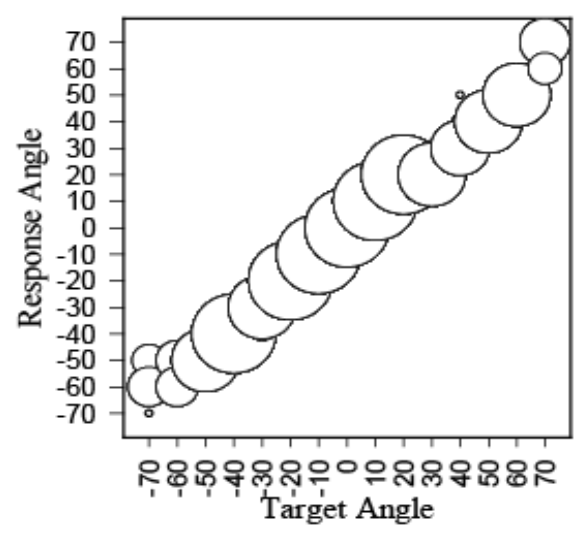

Figure B-23. Hearing Impaired Subject D perceived angle across each hearing aid condition plotted as a function of actual target angle of the stimuli. The bubble size indicates the number of times (out of ten trials) that a particular angle was chosen. Larger bubbles indicate an angle that was often perceived, whereas smaller bubbles indicate an angle was rarely perceived. 
HI Subject E; Open - Omnidirectional

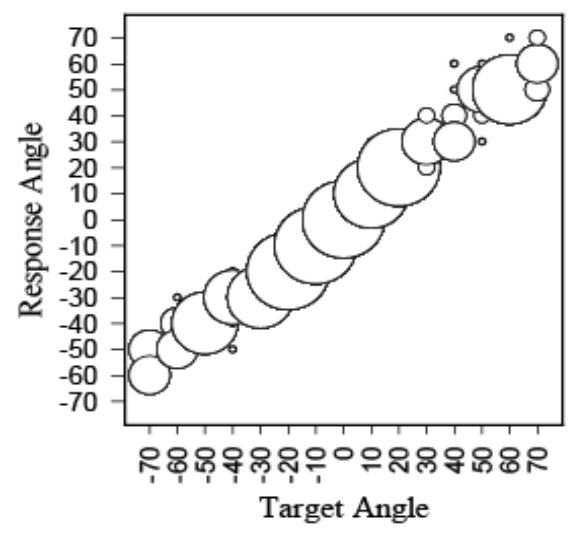

HI Subject E; Open - Adaptive Directional

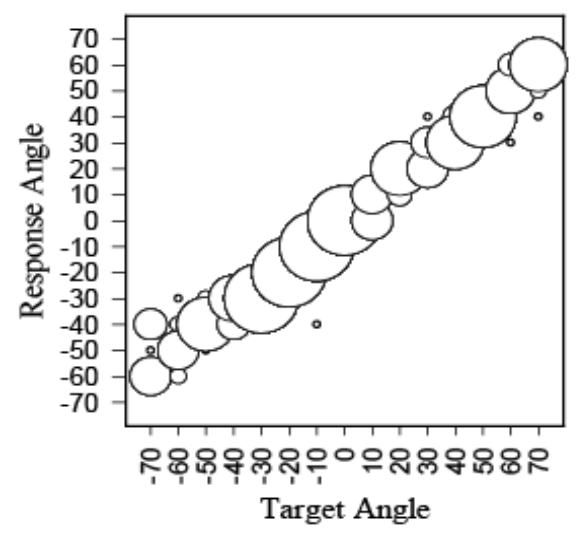

HI Subject E; Open - Fixed Directional

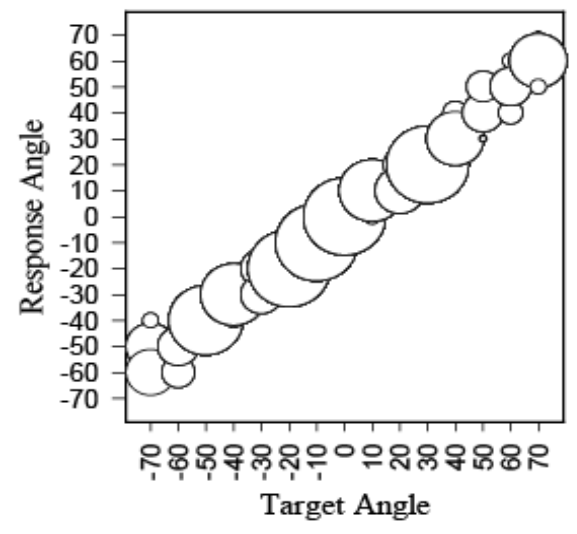

HI Subject E; Micro - Omnidirectional

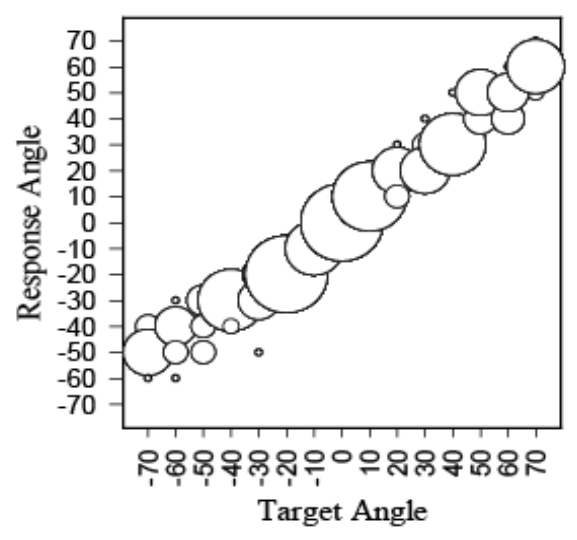

HI Subject E; Micro - Adaptive Directional

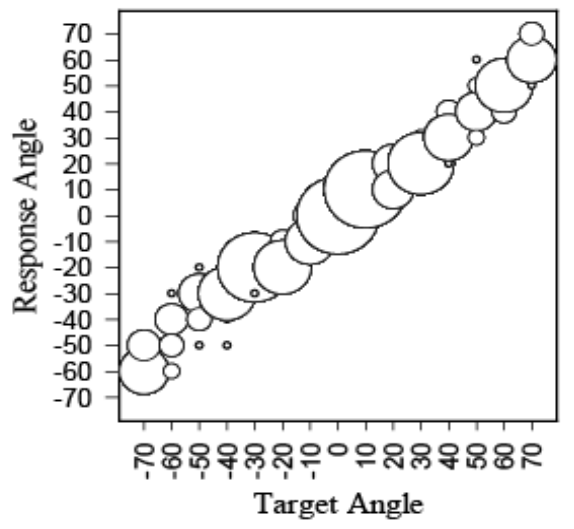

HI Subject E; Micro - Fixed Directional

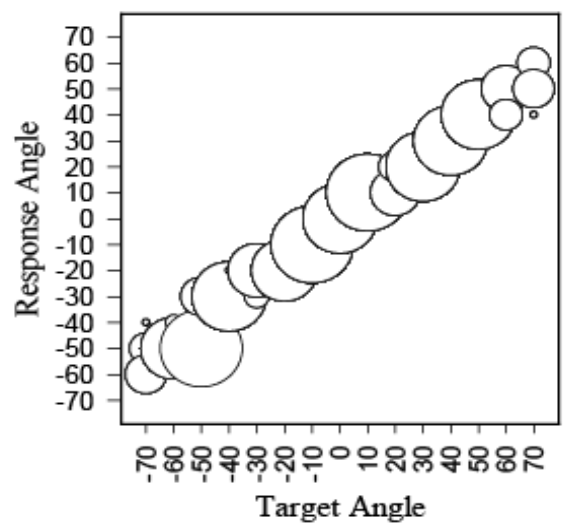

Figure B-24. Hearing Impaired Subject $\mathbf{E}$ perceived angle across each hearing aid condition plotted as a function of actual target angle of the stimuli. The bubble size indicates the number of times (out of ten trials) that a particular angle was chosen. Larger bubbles indicate an angle that was often perceived, whereas smaller bubbles indicate an angle was rarely perceived. 
HI Subject F; Open - Omnidirectional

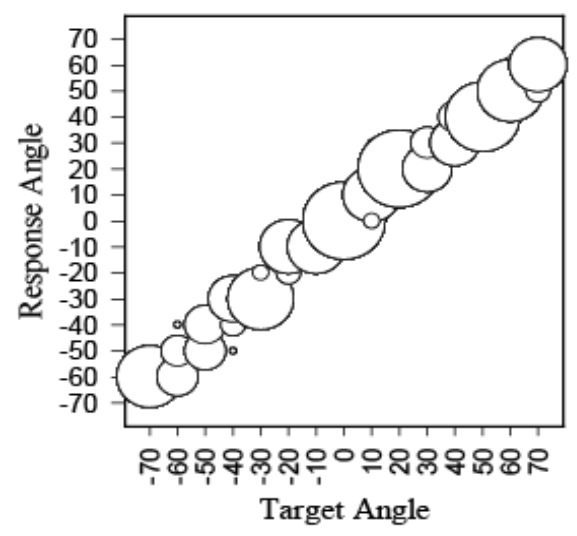

HI Subject F; Open - Adaptive Directional

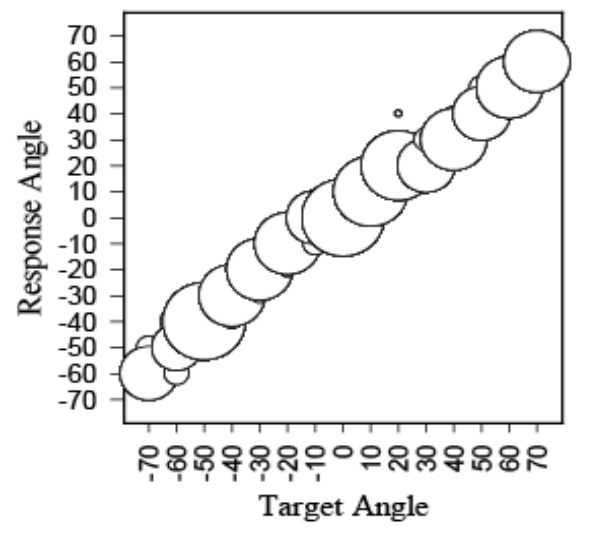

HISubject F; Open - Fixed Directional

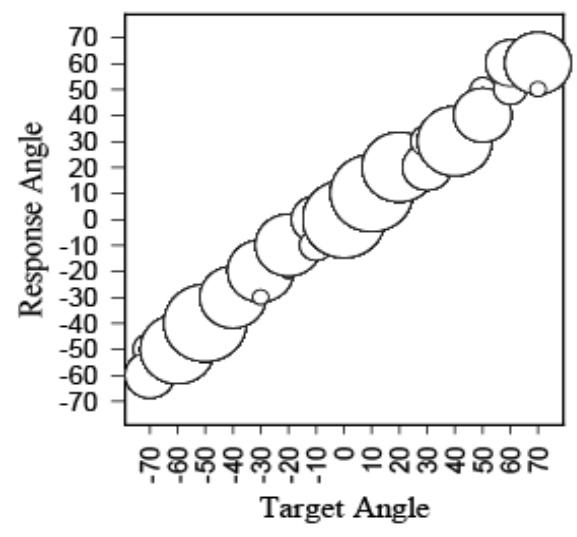

HI Subject F; Micro - Omnidirectional

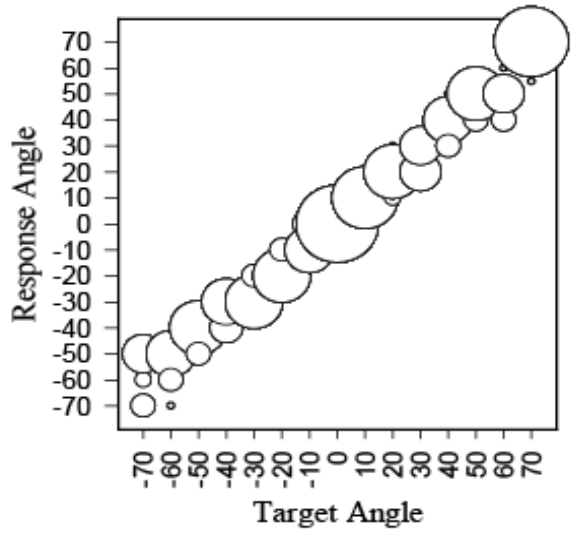

HI Subject F; Micro - Adaptive Directional

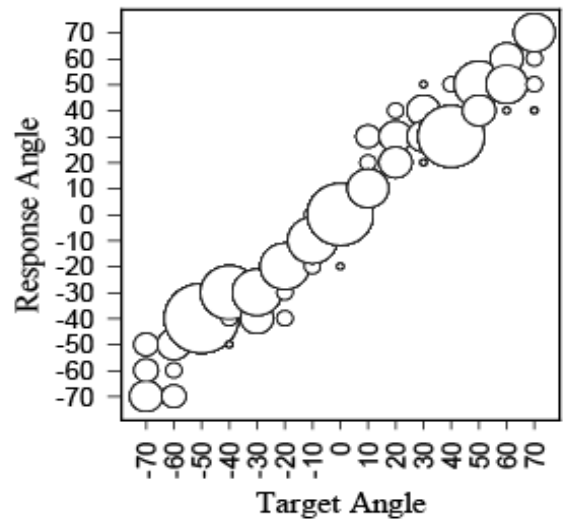

HI Subject F; Micro - Fixed Directional

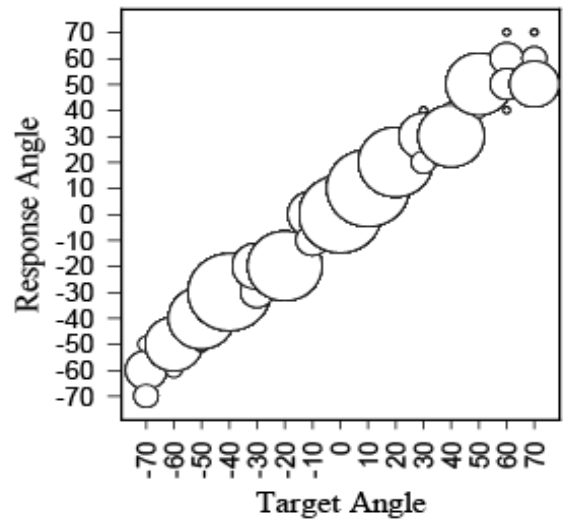

Figure B-25. Hearing Impaired Subject $F$ perceived angle across each hearing aid condition plotted as a function of actual target angle of the stimuli. The bubble size indicates the number of times (out of ten trials) that a particular angle was chosen. Larger bubbles indicate an angle that was often perceived, whereas smaller bubbles indicate an angle was rarely perceived. 
HI Subject G; Open - Omnidirectional

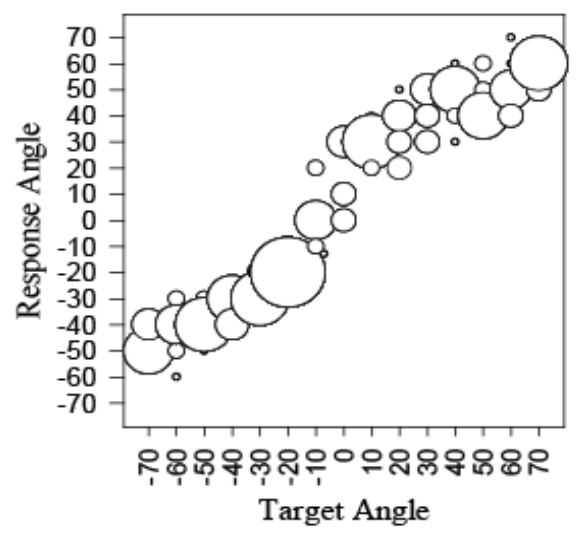

HI Subject G; Open - Adaptive Directional

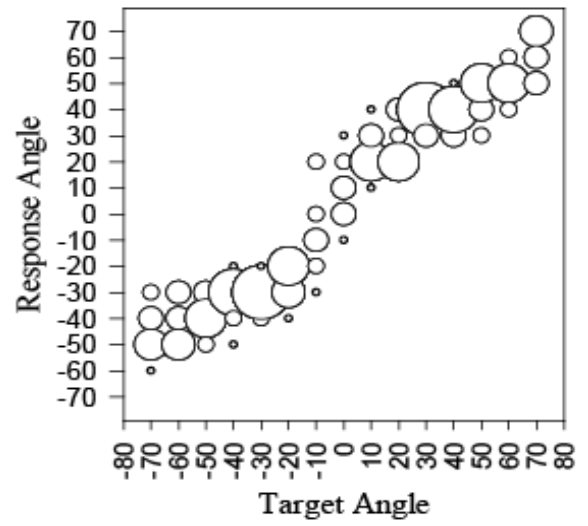

HI Subject G; Open - Fixed Directional

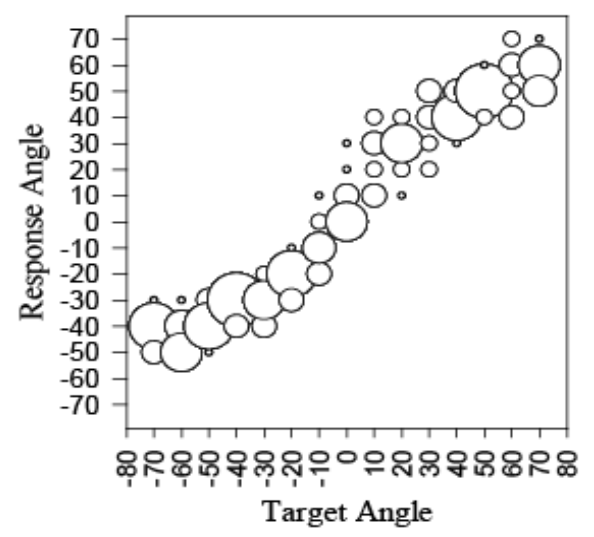

HI Subject G; Micro - Omnidirectional

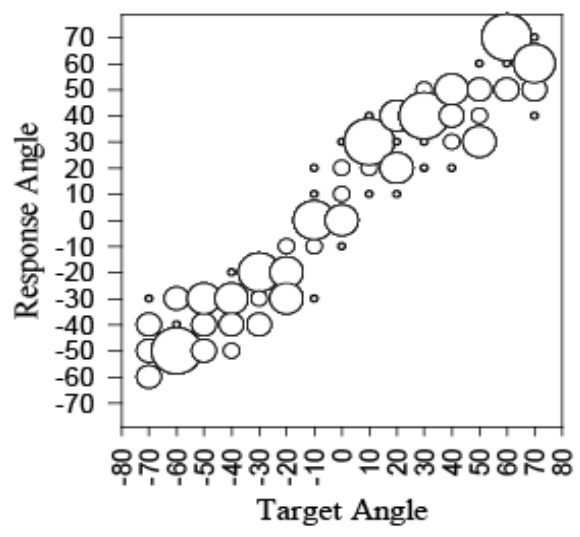

HI Subject G; Micro - Adaptive Directional

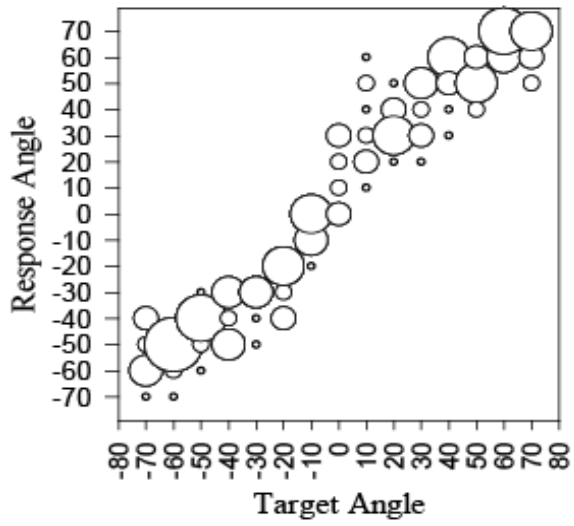

HI Subject G; Micro - Fixed Directional

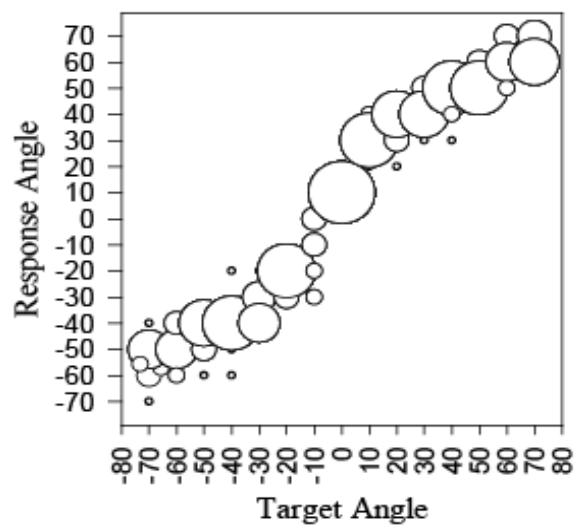

Figure B-26. Hearing Impaired Subject $G$ perceived angle across each hearing aid condition plotted as a function of actual target angle of the stimuli. The bubble size indicates the number of times (out of ten trials) that a particular angle was chosen. Larger bubbles indicate an angle that was often perceived, whereas smaller bubbles indicate an angle was rarely perceived. 
HI Subject H; Open - Omnidirectional

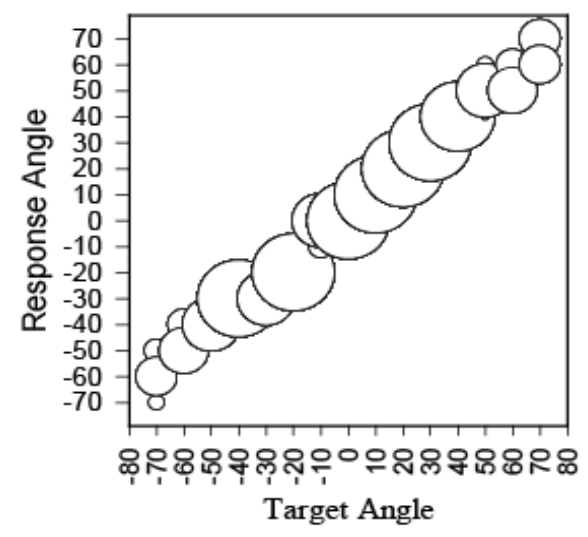

HI Subject H; Open - Adaptive Directional

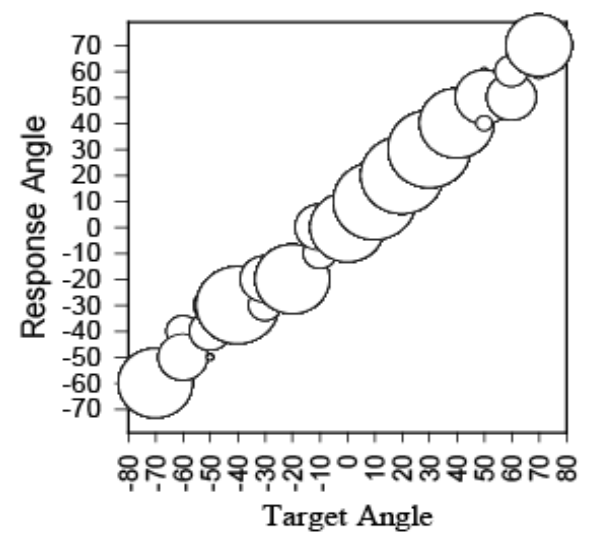

HI Subject H; Open - Fixed Directional

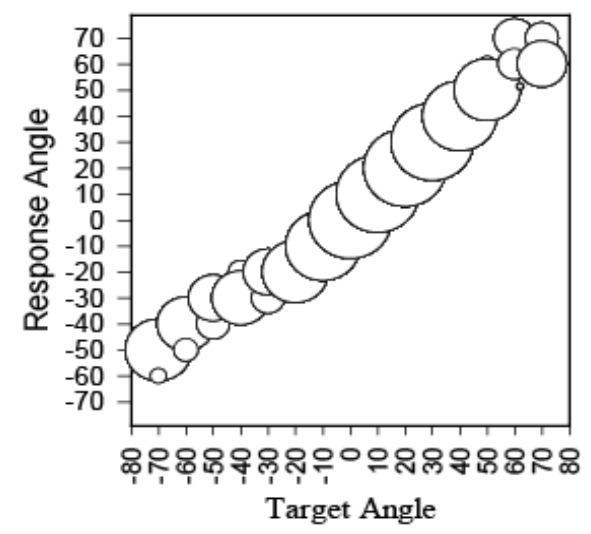

HI Subject H; Micro - Omnidirectional

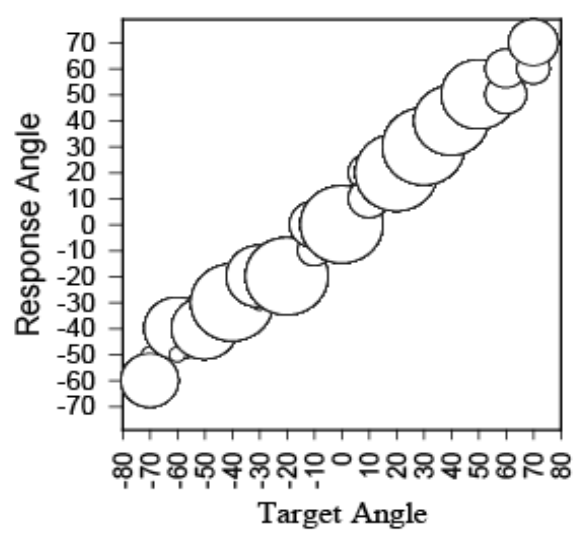

HI Subject H; Micro - Adaptive Directional

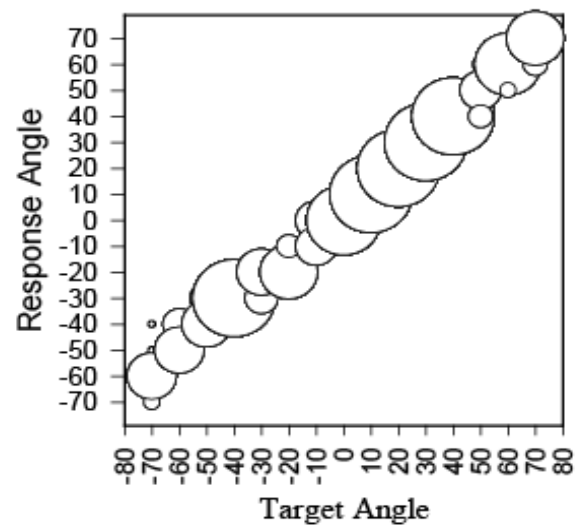

HI Subject H; Micro - Fixed Directional

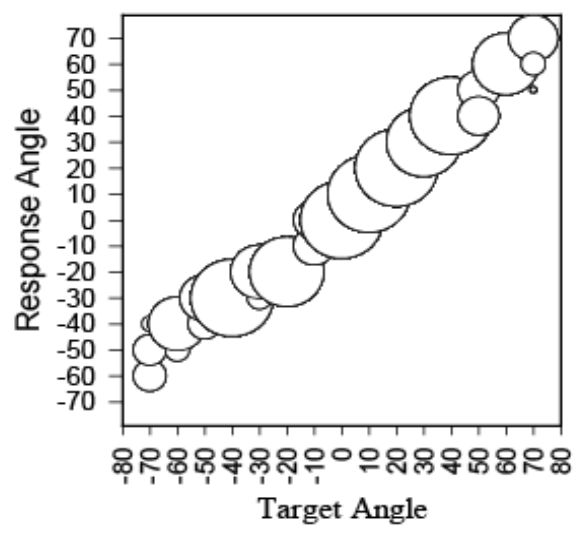

Figure B-27. Hearing Impaired Subject $H$ perceived angle across each hearing aid condition plotted as a function of actual target angle of the stimuli. The bubble size indicates the number of times (out of ten trials) that a particular angle was chosen. Larger bubbles indicate an angle that was often perceived, whereas smaller bubbles indicate an angle was rarely perceived. 
HI Subject I; Open - Omnidirectional

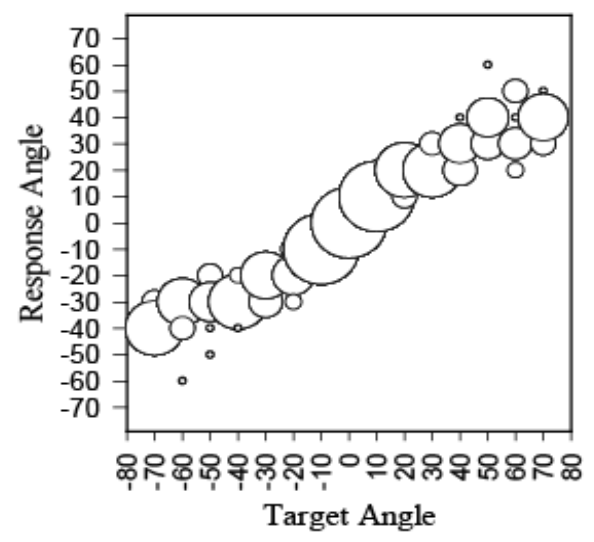

HI Subject I; Open - Adaptive Directional

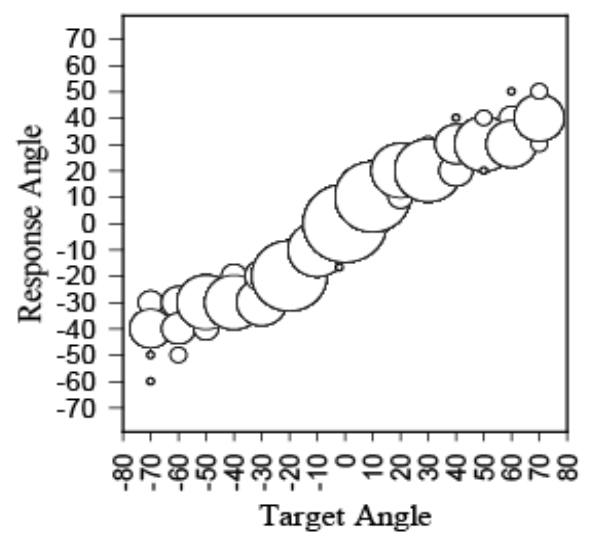

HI Subject I; Open - Fixed Directional

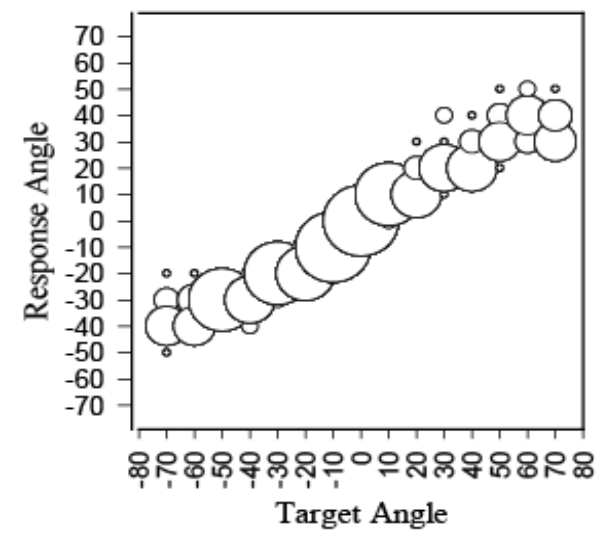

HI Subject I; Micro - Omnidirectional

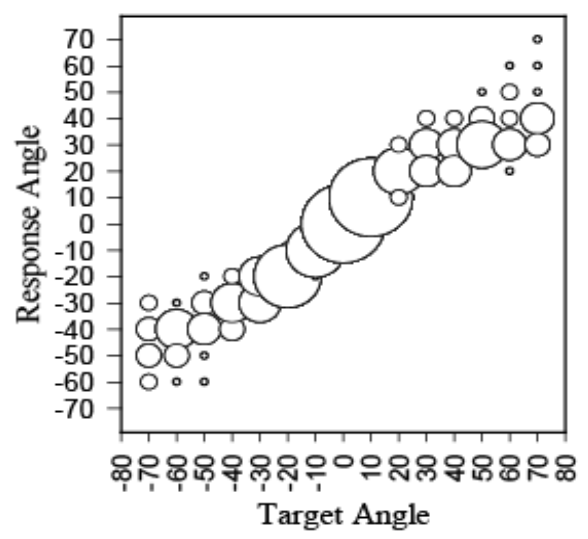

HI Subject I; Micro - Adaptive Directional

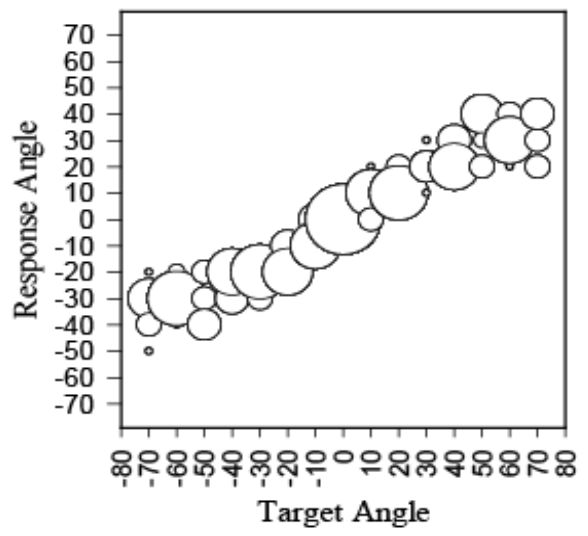

HI Subject I; Micro - Fixed Directional

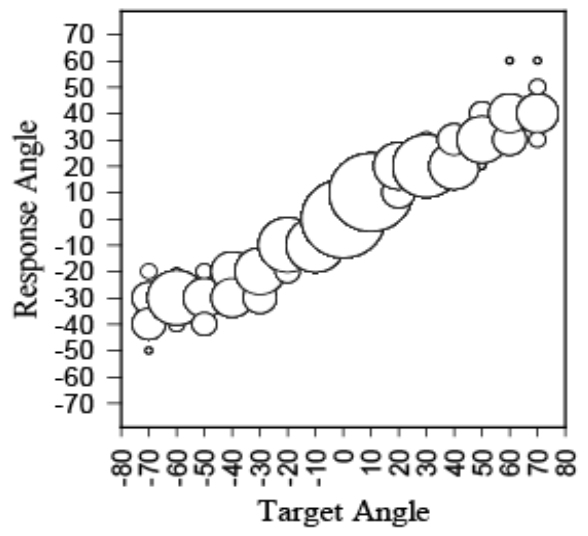

Figure B-28. Hearing Impaired Subject I perceived angle across each hearing aid condition plotted as a function of actual target angle of the stimuli. The bubble size indicates the number of times (out of ten trials) that a particular angle was chosen. Larger bubbles indicate an angle that was often perceived, whereas smaller bubbles indicate an angle was rarely perceived. 


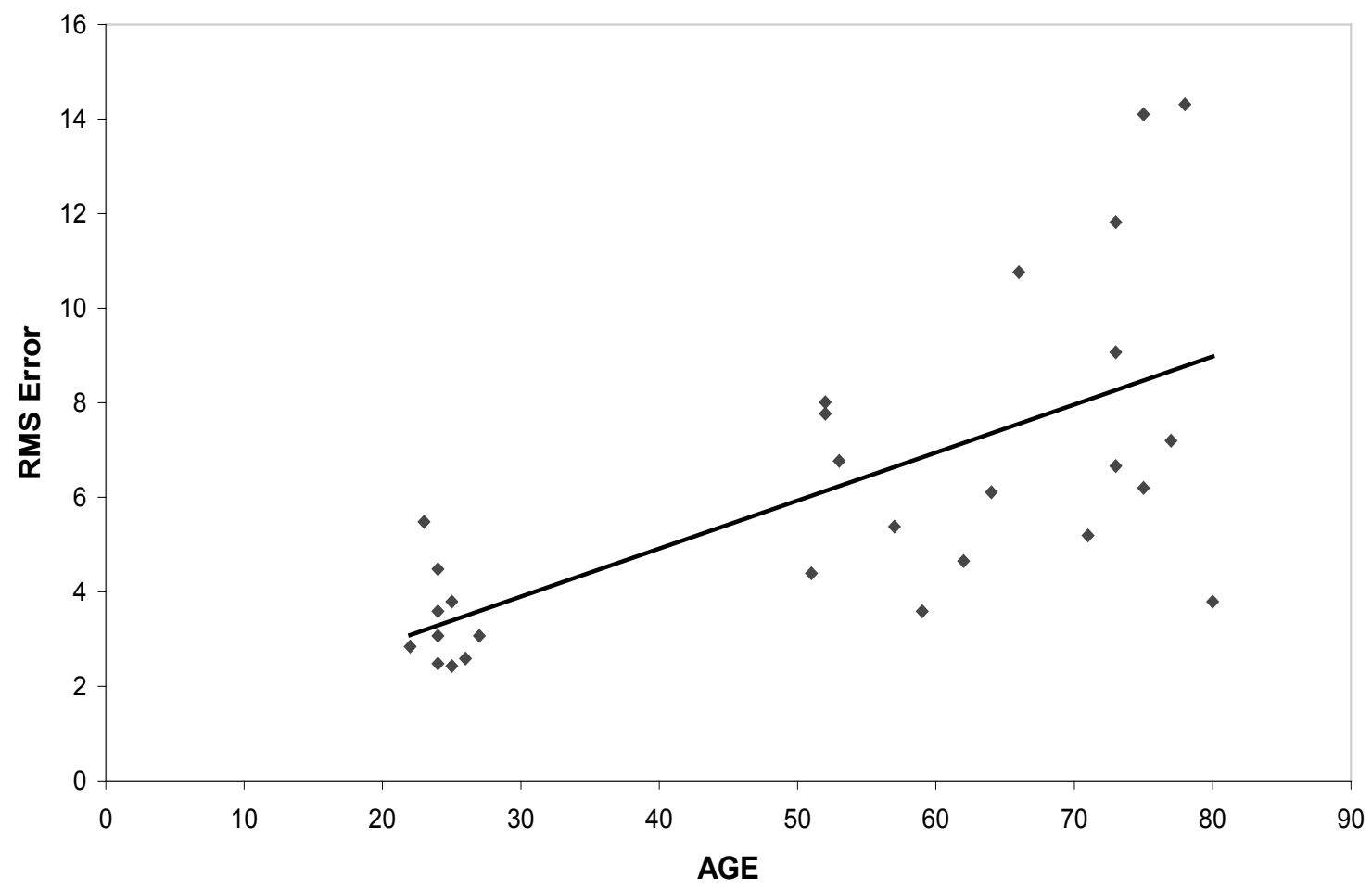

Figure B-29. Scatterplot displaying correlation between participant age and RMS error. 


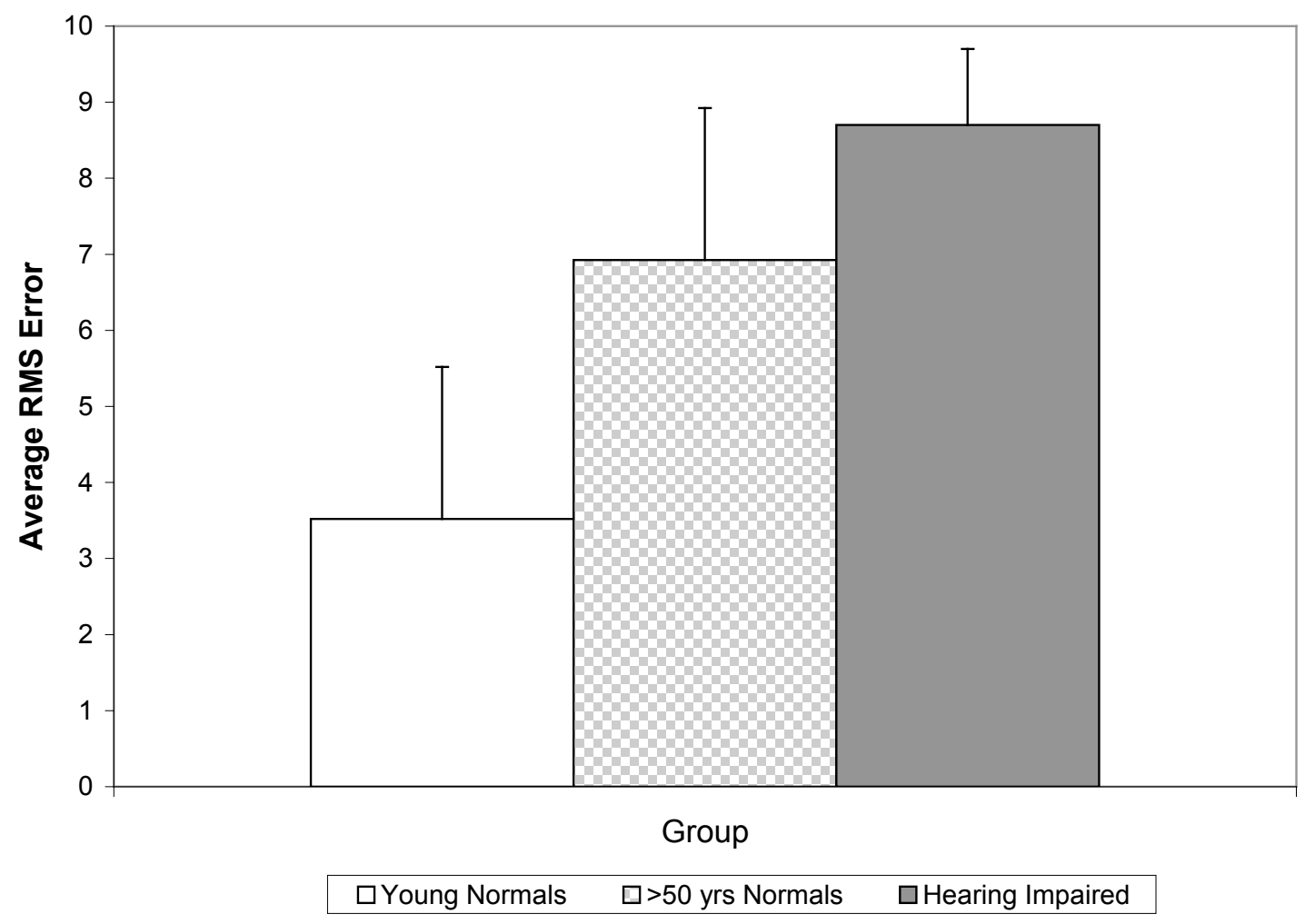

Figure B-30. Unaided/Unamplified performance by Group. 


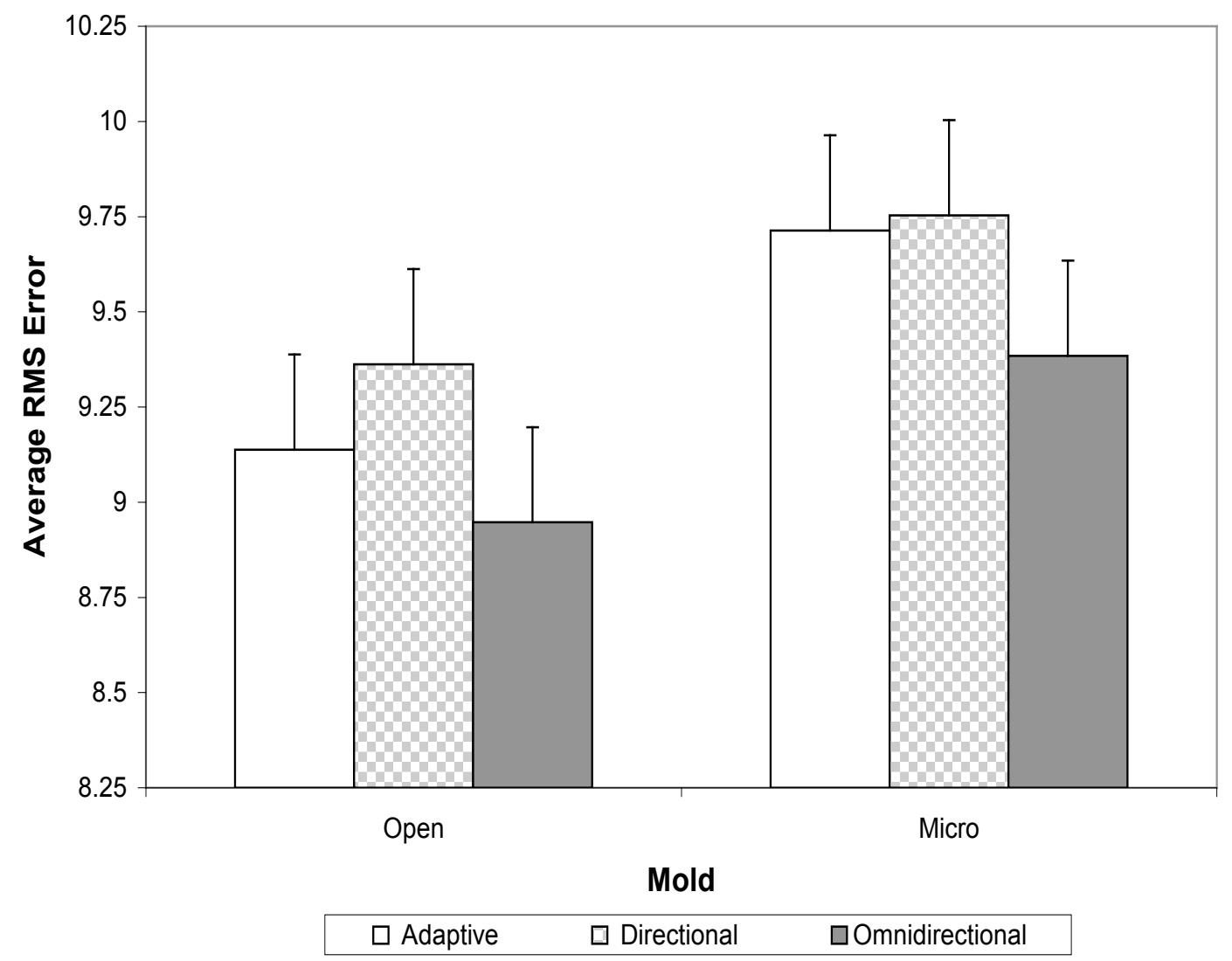

Figure B-31. Localization Acuity by Microphone Configuration within each Dome Condition. 
APPENDIX C. CONSENT FORM 


\section{Effect of Occlusion and Directionality on Horizontal Localization}

\author{
Principal Investigator: \\ Co-Investigator:
}

\author{
Lynzee N. Alworth, M.A.
}

Patrick N. Plyler, Ph.D.

\section{INTRODUCTION:}

You are being given the opportunity to participate in this research study. Research studies include only people who choose to take part. Please read this consent form carefully and take your time making your decision. As your study doctor or study staff discusses this consent form with you, please ask him/her to explain any words or information that you do not clearly understand. We encourage you to talk with your family and friends before you decide to take part in this research study. The nature of the study, risks, inconveniences, discomforts, and other important information about the study are listed below.

Please tell the study doctor or study staff if you are taking part in another research study.

The purpose of this study is to investigate how your localization ability to sounds may be affected by the use of hearing aids. More specifically, how different microphones and ear molds may affect your ability to localize a sound source. This study will also determine the effect of hearing loss on localization ability. Therefore, this study aims to answer two specific questions: (1): Does hearing loss affect a listener's ability to localize sound? (2): Do new ear mold designs and microphones affect a listener's ability to localize sound?

Approximately 40 subjects will be participating in this study and all subjects will be participating locally.

The study will take place at The University of Tennessee's Hearing and Speech Center (Audiology Clinic/Labs) in Knoxville, Tennessee.

Your participation in this study will last approximately 1-2 hours.

\section{PROCEDURES TO BE FOLLOWED:}

You will receive an interview conducted by the Principal Investigator. During this interview, 1) a verbal description of the study will be given; including the general purpose, nature of participation, and potential risks and benefits, and 2) the written consent form will be read and signed by you if you wish to participate. You will then receive a hearing evaluation, which will include otoscopy (visual examination of the external ear) and audiometric (hearing test across several frequencies/pitches of sound) testing. The audiometric testing will occur in a sound treated booth and stimuli will be delivered through insert earphones 
(earplugs). Each procedure performed during the audiometric testing is routinely performed at the University of Tennessee Audiology Clinic in the assessment of hearing sensitivity. Each qualification session (hearing test) will take approximately thirty minutes. If you do not meeting the qualification criteria for further participation in the study you will not be required to complete the localization task. If you qualify for participation in the study you will complete a localization task. This task occurs in a sound treated booth with several speakers and a computer screen. You will be asked to identify the direction/location of sounds you will hear.

Day 1/Visit 1:

- Interview with Principal Investigator

- Hearing Evaluation (qualification session)

Day 2/Visit 2 (if qualified):

- Hearing aid fitting

- Horizontal localization task

\section{RISKS ASSOCIATED WITH PARTICIPATION:}

There are no known psychological, social, or legal risks or side effects involved with participation of the proposed project. All testing conducted in this study is used on a daily basis in audiological clinics and research laboratories. There are no risks of noise-induced hearing loss to you because stimuli level will not exceed average conversational levels (68 dB SPL). Although it is not expected, you will be asked to inform the investigator immediately if they experience discomfort of any kind during the course of the experiment. Though no known physical risks exist there are, however, there is the potential risk of loss of confidentiality. Every effort will be made to keep your information confidential; however, this cannot be guaranteed. Confidentiality will be maintained by assigning all participants a letter and numeric code that will be used on all computer and data files. There will be no way to associate the name of the individual subject with her or her numeric code by looking at either code or name alone. A single document will list the names of the participants with their codes. This will be kept in a locked file cabinet in the applicant's office. This method has been used to protect confidentiality during prior experiments.

\section{BENEFITS ASSOCIATED WITH PARTICIPATION:}

There are no direct benefits of participation within this study.

\section{ALTERNATIVES TO PARTICIPATION:}

Treatments for hearing loss, other than those being evaluated in this study are available. Your physician and/or audiologist can discuss treatment options with you. You will not have to undergo a hearing evaluation if you do not participate in this study. 


\section{CONFIDENTIALITY:}

All your paper research records will be stored in locked file cabinets and will be accessible only to research personnel. All your electronic research records will be computer password protected and accessible only to research personnel.

Your localization ability sample will be maintained in the research lab during the study and will be labeled with a code. A master key which links your name with the code on your localization data will be maintained at the local investigative site.

Under federal privacy regulations, you have the right to determine who has access to your personal health information (called "protected health information" or PHI). PHI collected in this study may include your medical history, the results of physical exams, lab tests, x-ray exams, and other diagnostic and treatment procedures, as well as basic demographic information. Your PHI may also be shared with, which sponsors and provides funds for this research; which has been hired by the sponsor to coordinate the study; and a Data and Safety Monitoring Committee. However, these latter organizations may not have the same obligations to protect your PHI. The Institutional Review Board (IRB) at the University of Tennessee Health Science Center may review your PHI as part of its responsibility to protect the rights and welfare of research subjects. Your PHI will not be used or disclosed to any other person or entity, except as required by law, or for authorized oversight of this research study by other regulatory agencies, or for other research for which the use and disclosure of your PHI has been approved by the IRB. Your PHI will be used only for the research purposes described in the Introduction of this consent form. Your PHI will be used until the study is completed for as long as the sponsor reports study data to the FDA indefinitely.

You may cancel this authorization in writing at any time by contacting the principal investigator listed on the first page of the consent form. If you cancel the authorization, continued use of your PHI is permitted if it was obtained before the cancellation and its use is necessary in completing the research. However, PHI collected after your cancellation may not be used in the study. If you refuse to provide this authorization, you will not be able to participate in the research study. If you cancel the authorization, then you will be withdrawn from the study. Finally, the federal regulations allow you to obtain access to your PHI collected or used in this study

Information about your participation in this study or the results of procedures performed in this study will be placed in your medical record; as such, this information could be made available to your employer or insurer.

You will not be identified in any presentations or publications based on the results of this research study. 


\section{COMPENSATION AND TREATMENT FOR INJURY:}

You understand that you are not waiving any legal rights or releasing the University of Tennessee, from liability for negligence. You also understand that in the event of physical injury resulting from research procedures, that the University of Tennessee does not have funds budgeted for compensation either for lost wages or for medical treatment. Therefore, the University of Tennessee does not provide for treatment or reimbursement for such injuries.

If you suffer a research related injury, your study doctor will provide acute medical treatment and will provide you with a subsequent referral to appropriate health care facilities.

You and/or your insurance carrier will be billed for the costs associated with the medical treatment of a research related injury.

\section{QUESTIONS:}

If you have any questions about this research study you may contact Lynzee Alworth at 865-974-1571.

In the event of a research related injury, contact Patrick Plyler at 865-974-7588.

You may contact Dr. Terrence F. Ackerman, Ph.D., UTHSC IRB Chairman at 901-448-4824 if you have any questions about your rights as a participant in this study or your rights as a research subject.

\section{PAYMENT FOR PARTICIPATION:}

You will be given a free hearing evaluation in exchange for participation in this study. The scientific and clinical communities will benefit from a better understanding of the effects of occlusion and directionality on horizontal localization ability of the listener.

\section{COSTS OF PARTICIPATION:}

The hearing evaluation at the initial appointment will be provided to you free of charge.

\section{PREMATURE TERMINATION:}

Your participation in this research study may be terminated by the investigator without regard to your consent for the following reasons:

- Insufficient/inappropriate hearing aid fitting dependent on hearing loss

\section{VOLUNTARY PARTICIPATION:}

Your participation in this research study is voluntary and your refusal to participate or your decision to withdraw will involve no penalty or loss of benefits to which you are otherwise entitled. 
If you decide to stop being part of the study, you should tell your study doctor. In addition, any information that you have already provided will be kept in a confidential manner.

\section{CONSENT OF SUBJECT:}

You have read or have had read to you a description of the research study as outlined above. The investigator or his/her representative has explained the study to you and has answered all the questions you have at this time. You knowingly and freely choose to participate in the study. A copy of this consent form will be given to you for your records.

Signature of Research Subject

Date

Time

Printed Name of Research Subject

Signature of Person Obtaining Consent

Date

Time

\section{Printed Name of Person Obtaining Consent}

In my judgment, the subject or the legally authorized representative has voluntarily and knowingly given informed consent and possesses the legal capacity to give informed consent to participate in this research study. 


\section{VITA}

Lynzee Nicole Alworth was born in Butler, Pennsylvania in 1983. She graduated high school from Allegheny-Clarion Valley in 2001 and continued her education at Juniata College, receiving a Bachelor of Science degree in Human Development and Psychology in 2005. Lynzee then decided to pursue her professional degree at the University of Tennessee, where she received her Master of Arts in Audiology in 2007 and her Doctor of Philosophy in Speech and Hearing Science in 2010. Lynzee's research has concentrated on new amplification technologies and has one article published within the area. She has had 7 presentations at national conferences, 2 presentations at state conferences in which she was an award winner, received 5 scholarships and participated in 1 fellowship. Lynzee has also been an instructor for Audiology II, Pediatric Audiology for non-majors, and Hearing Disorders at the University of Tennessee. 